\title{
Chick embryo experimental platform for 3D tissue engineering modelling of cancer micrometastases for tumor biology, drug development and nanomaterials testing
}

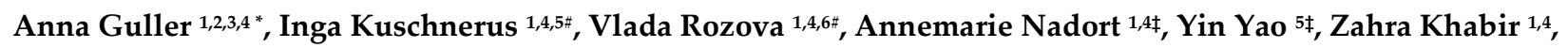 \\ Alfonso Garcia-Bennett ${ }^{1,4}$, Liuen (Olivia) Liang ${ }^{1,4}$, Aleksandra Polikarpova ${ }^{4,5,7}$, Yi Qian ${ }^{2}$, Ewa M. Goldys ${ }^{1,4,5^{*}}$ and \\ Andrei V. Zvyagin $1,4,8,9^{*}$
}

\footnotetext{
${ }^{1}$ Faculty of Science and Engineering, Macquarie University, Sydney, NSW 2109, Australia;

2 Faculty of Medicine, Health and Human Sciences, Macquarie University, Sydney, NSW 2109, Australia;

${ }^{3}$ Institute for Regenerative Medicine, Sechenov First Moscow State Medical University (Sechenov University), Moscow, 119991, Russia;

${ }^{4}$ ARC Centre of Excellence for Nanoscale Biophotonics, Australia;

${ }_{5}^{5}$ University of New South Wales, Sydney, NSW 2052, Australia;

${ }^{6}$ Institute for Biology and Biomedicine, Lobachevsky State University, Nizhny Novgorod, 603950, Russia;

${ }^{7}$ Westmead Hospital, Westmead, NSW 2145, Australia;

8 Centre of Biomedical Engineering, Sechenov First Moscow State Medical University (Sechenov University),

Moscow, 119991, Russia;

9 Laboratory of NanoOncoTheranostics, Institute of BioOrganic Chemistry, RAS; Moscow, 117997, Russia.
}

$\#$ \# Equally contributed co-authors. * Correspondence: anna.guller@,q.edu.au (A.G.), e.goldys@unsw.edu.au (E.G.), andrei.zvyagin@mq.edu.au (A.Z.)

\begin{abstract}
Colonization of distant organs by tumor cells is a critical step of cancer progression. The initial avascular stage of this process (micrometastasis) remains almost inaccessible to study due to the lack of relevant experimental approaches. Here, we introduce an in vitro/in vivo model of organspecific micrometastases of triple-negative breast cancer (TNBC) that is fully implemented in a costefficient chick embryo (CE) experimental platform. The model is built as three-dimensional (3D) tissue engineering constructs (TECs) combining human MDA-MB-231 cells and decellularized CE organ-specific scaffolds. TNBC cells colonized CE organ-specific scaffolds in 2-3 weeks, forming tissue-like structures. The feasibility of this methodology for basic cancer research, drug development and nanomedicine was demonstrated on a model of hepatic micrometastasis of TNBC. We revealed that MDA-MB-231 differentially colonize parenchymal and stromal compartments of the liver-specific extracellular matrix (LS-ECM) and become more resistant to the treatment with molecular Doxorubicin (Dox) and Dox-loaded mesoporous silica nanoparticles than in monolayer cultures. When grafted on CE chorioallantoic membrane, LS-ECM-based TECs induced angiogenic switch. These findings may have important implications for the diagnosis and treatment of TNBC. The methodology established here is scalable and adaptable for pharmacological testing and cancer biology research of various metastatic and primary tumors.
\end{abstract}

Keywords: chick embryo; 3D culture; tumor models in vitro; tissue engineering; metastasis; micrometastasis; triple-negative breast cancer; liver; mesoporous silica nanoparticles; doxorubicin

\section{Introduction}

Metastases remain the leading cause of cancer-related deaths and one of the biggest challenges in oncology [1,2]. During the metastatic cascade, cancer cells shed from the primary tumor, travel through the blood and lymphatic vessels, and, following the escape from the vasculature (extravasation), may attach (arrest/homing) and form a colony in a secondary organ [3]. This colony of the disseminated cancer cells may further develop into 
avascular micrometastasis and, if not destroyed by immune system, either stay dormant or progress to massive and blood-perfused macrometastases $[4,5]$ through the tumor-induced acceleration of blood vessels growth in the vicinity of the cancer cell colony (angiogenic switch) [6]. In contrast to macrometastases, the early stages of metastatic cascade, from homing to angiogenic switch, are extremely difficult to detect in natural conditions and to study in the lab, primarily due to their asymptomatic course, small size, which is below the detection limit of non-invasive methods, and sparse distribution in the body that can be easily missed even by histology [7-9]. As a result, the studies of the latent, early-stage post-extravasation events like cancer cells' homing and the initial, avascular phase of the metastatic colonization of secondary organs are rare [5] and the most experimentally challenging $[8,10]$. It is also difficult to dope out one of the biggest mysteries in cancer biology, such as preferential metastatic organotropism of certain types of tumors $[3,11,12]$, well-known by Paget's "seed and soil" metaphor. Therefore, addressing the problem of micrometastases is on demand and requires the creation of robust experimental models for the development of organ-specific diagnostic and treatment strategies and improvement of the survival rates in patients with metastatic cancers $[13,14]$.

Importantly, after the extravasation, cancer cells enter the extracellular domain of the distant organs [15]. Then, colonization of the secondary organs is particularly affected by the interactions between the invading cancer cells and the resident perivascular and parenchymal extracellular matrix (ECM) [12]. There is a growing body of literature indicating the major role of ECM composition and physical properties in controlling of tumor progression (see [16] for a comprehensive review). Availability and size of the cellular adhesion sites (as a function of ECM composition and architecture) and the stiffness [17], as well as confinement and the porosity $[18,19]$ are among the factors that significantly contribute to this regulation. However, the biochemical and physical parameters of ECM are tightly coupled, and the specific contribution of the individual factors in cancer cells' dormancy, proliferation, and invasion can be defined in artificial reductionistic systems only with a notable chance of experimental bias. Therefore, we think, that biologically relevant models of micrometastases should include the cancer cells together with the native organ-specific ECM. This is potentially achievable in animal models of spontaneous metastases where the cancer cells are injected into the blood stream or immediately into the host organ. However, while common for the macrometastatic modelling, this approach is not particularly suitable for the studies of micrometastases, especially for the drug development-focused research, due to the low throughput capacity and high cost. In parallel, conventional monolayer in vitro models are unable to reflect the micrometastatic biology, while the most known three-dimensional (3D) cell culture systems like multicellular spheroids are missing the role of the ECM as the homing niche [20].

Tissue engineering provides a useful tool for the reconstruction of the complex organ- and disease-specific environments by culturing cells in vitro on the scaffolds obtained by a special procedure named decellularization (DCL). DCL involves removal of the cells from the native organs, tissues or cultured cell sheets [21-25]. Decellularized tissues are among the most clinically successful biomaterials in regenerative medicine and reconstructive surgery because, ideally, they can preserve three essential organ-specific features of ECM: the composition, the architecture and the biomechanical properties [26]. Tumor models on decellularized scaffolds are 3D in vitro systems that hold a great promise for cancer biology research, drug development and nanomedicine [27-29].

It is generally held that the best quality of DCL (implying low level of cellular residuals and minimally affected ECM [30]) can be achieved by perfusion of the whole organs via natural vasculature (whole-organ-DCL, or WO-DCL) in a bioreactor [24,31] or, possibly, in situ [32,33]. This procedure requires precise surgical technique, very careful cannulation of the major blood vessels in a live anesthetized animal. It is followed by the ex vivo processing/preparation of the cannulated and extirpated organ or organ complexes. Unfortunately, and inevitably, WO-DCL is almost non-scalable, low-reproducible, and therefore non-applicable in high-throughput, e.g., pharmacological or nanomedical, re- 
search. Alternatively, small fragments of organs and tissues can be decellularized by immersion in static or shaking baths with the solutions able to destroy cellular membranes (mostly hypotonic salts and various types of detergents) and remove the cellular debris from the tissue (mostly with water and buffers) [25]. This is a well-established technology that is widely used in preparation of highly biocompatible collagen-based biomaterials for clinical applications (as surgical plastics materials and acellular grafts, temporary wound dressings, or vehicles for the drugs, bioactive factors or cells) [34]. However, for the experimental use, further processing of the decellularized material obtained by this simplified method is usually required to achieve reproducibility and scalability of the scaffolds production. For example, freeze drying, preparation of gels, bioinks and composites are the popular ways to make bioengineering products from the tissues decellularized by immersion protocols [34-37]. While transforming the decellularized tissues in manufacturable materials, such processing destroys the organ- or tissue-specific architecture of ECM, converting the native scaffold in an artificial substrate.

In addition, sourcing of the tissues for DCL remains an ethical, economical and biosafety problem. In regenerative medicine, human cadaveric and discarded organs and tissues are the preferred but low available materials for the preparation of scaffolds by DCL [30]. As ECM proteins are highly evolutionary conservative and therefore low immunogenic [38-40], there is an option to use animal organs and tissues, for example, from cows and pigs, for this purpose. However, the risk of transmission of infections via decellularized materials and induction of extensive foreign body response remains one of the limitations for the applications of not heavily-processed human and animal tissues $[30,35,41,42]$.

In the current study, we wanted to bypass the indicated controversies, reconcile the advantages of WO-DCL and immersion DCL methods and find a good source of organs and tissues for DCL that would allow ethical, feasible, labor- and cost-efficient modelling of tumor micrometastases for both cancer biology research and drug/nanomedicine development. Here, we propose a new approach relying on the use of industry supplied poultry product such as chick embryos (CE) of late embryonic age as a highly controllable, reproducible and affordable source of the organs for DCL. Relying on a small size of the CE organs, we developed an immersion DCL protocol applicable for the efficient DCL of the whole organs, which we named iWO-DCL ("immersion-agitation whole-organ DCL"). Next, we adapted the iWO-DCL protocol for the creation of the acellular organspecific scaffolds (AOSS) of several CE organs, including brain, lungs, liver, heart, small intestine, proventriculus and ventriculus, breast muscle, and spleen. The protocol involves the use the same set of solutions at room temperature with adjustable immersion conditions and does not require complex equipment or high-level surgical skills. We recellularized the obtained panel of AOSS with the cells of one of the most metastatically aggressive human tumors, the triple-negative breast cancer (TNBC), cultured the obtained three-dimensional (3D) tissue engineering constructs (TECs) in vitro to reconstruct the situation of metastatic cells' arrest in the secondary organ and formation of the avascular micrometastases. The TECs were used for the analysis of the role of organ-specific ECM in the metastatic organotropism of TNBC, and the mechanisms of the colonization of different organs (with the main attention to the liver metastases). By grafting of the TECs that combined the CE liver AOSS and TNBC cells on chick embryo chorioallantoic membrane (CAM), we confirmed that the TEC tumor models can induce angiogenic switch in vivo, indicating the biological accuracy of the model such as the potential for the transformation into the vascularized macrometastases. Finally, we evaluated the feasibility of the 3D tissue engineered model of TNBC hepatic micrometastasis as a testbed for drug and nanomedicine development applications. 


\section{Materials and Methods}

\subsection{Study design}

The current study includes two series of experiments (Figure 1).

In the first series, we developed a protocol of DCL that is applicable to multiple chick embryo (CE) whole organs, tested the in vitro biocompatibility of the obtained acellular organ-specific scaffolds (AOSS) by seeding them with human triple-negative breast cancer (TNBC) cells. Next, to explore the "seeds and soil" hypothesis regarding the contribution of organ-specific ECM in the preferential metastatic spreading of breast cancer cells to certain organs, we applied digital analysis of the histological images to examine and quantify the organ-specific patterns of cellular attachment and colonization of the AOSS.

The second stage of the study was focused on the interactions between TNBC cells and liver-specific ECM presented as CE liver-derived AOSS. This experimental series included evaluation of the feasibility of the proposed 3D tissue engineering model of TNBC micrometastasis to the liver for cancer biology research and for drug and nanomedicines testing. The detailed design of the second series of experiments is presented in the Section A1 and Figure A1 (Appendix A).

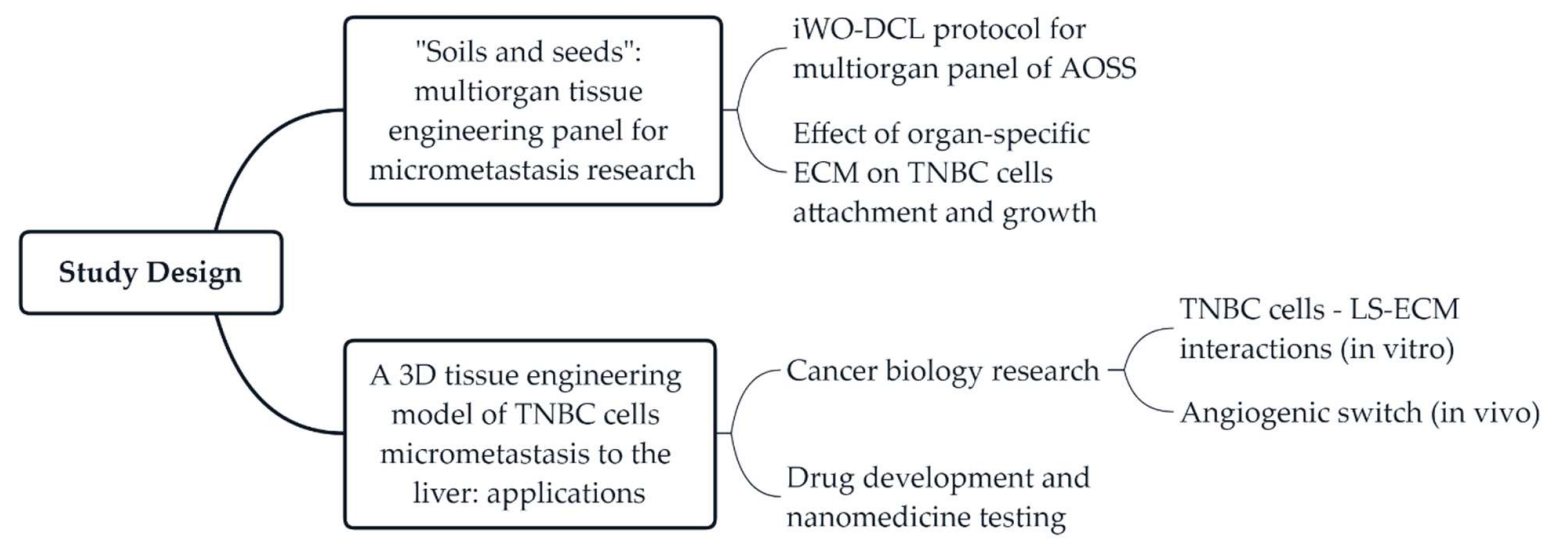

Figure 1. The design of the current study. Abbreviations: iWO-DCL - immersion whole organ decellularization, AOSS acellular organ-specific scaffolds, ECM - extracellular matrix, TNBC - triple-negative breast cancer, 3D three-dimensional, LS-ECM - liver-specific ECM.

\subsection{Chick embryo incubation and organ collection}

The study was approved by the animal ethics committee protocols of Macquarie University (ARA \# 2015/006) and The University of New South Wales (ACEC \# 19/103B). Fertilized chicken (Gallus gallus domesticus, the broiler breed Ross 308) eggs were delivered from a local hatchery. After the delivery, the eggs were allowed to rest for 3-4 $\mathrm{h}$ at room temperature and then incubated in a standard cradle-type laboratory poultry incubator (R Com MARU Max 190, Autoelex Co., LTD, South Korea) at $37.5{ }^{\circ}$ C, $65-70 \%$ humidity with hourly turn over until the embryonic day 18 (ED18), 3 days before natural hatching. This period was sufficient for the histoanatomical development of the organ structure of a chick. The pain sensitivity was immature at this stage, and it was permissible to extract an embryo from the egg for research purposes in accordance with the Australian code of animal research. On ED18, eggshells were opened by a cut on the blunt ends of eggs, and the embryo with embryonic membranes were extracted using forceps and immediately decapitated (this method of culling is proven to be less severe and keep the high quality of the tissues of the chicks [43]). Then feathers were removed from the abdominal wall and thorax, and the organs of interest were carefully extracted through the wide central section. The animal procedure used for angiogenic assay on the CE chorioallantoic membrane (CAM) is described below in the Section 2.6 and in the Appendix A (section A2.5). 


\subsection{Immersion whole-organ decellularization ( $i W O-D C L$ )}

The iWO-DCL was performed by our original immersion DCL method [44,45] with some modifications. The extracted chick embryo (CE) organs, including liver, lungs, heart, ventriculus and proventriculus, brain, small intestine, spleen, breast muscles, and skin, were washed in sterile PBS and placed in 50-mL Falcone tubes, 5-15 organs/ per tube (depending on the organ's size, for example, up to 5 livers per tube) filled with $35 \mathrm{~mL}$ of $0.1 \%$ solution of sodium dodecyl sulphate (SDS) in phosphate buffer saline (PBS), then closed tightly and fixed horizontally on the platform of an orbital shaker. Then the organs underwent shaking at the speed of 90-150 rotations per minute (rpm) with periodic aseptic changes of washing media for a fresh portion every $3 \mathrm{~h}$ during first $12 \mathrm{~h}$, and then every $6 \mathrm{~h}$ during the next $12 \mathrm{~h}$. Afterwards, the solution was changed daily until the organs became translucent, and the liquid media turned colorless and transparent. The total processing time ranged from 2 to 21 days depending on the organ (approximately, 14-21 days for the livers, and 2-4 days for smaller organs) and the embryo flock. Next, the processed organs were aseptically placed in sterile containers and washed with $1 \%$ of antibiotic-antimycotic solution (A-A) (\#A5955, Sigma-Aldrich) in PBS (pH 7.0) for under shaking (30 $90 \mathrm{rpm}$ ) with periodical changing of A-A/PBS with fresh portions, until the washing media became transparent, colorless, with no observable tissue components and foam. The processing was performed at room temperature. The overview and the technical notes explaining further details of the iWO-DCL protocol are shown in the Appendix A, Table A1 and in Supplementary Video 1.

As-obtained scaffolds were stored in fresh sterile 1\% A-A/PBS solution in a fridge $\left(+4{ }^{\circ} \mathrm{C}\right)$ until further use and preserved their functionality as 3D culture substrates for at least 1 year. The iWO-DCL protocol presented here has been tested for the Ross 308, Cobb 500, and several Rhode Island $\times$ White Leghorn crossbreed CEs (on at least a hundred eggs of each type). The best and robust performance was achieved when using the broiler breeds (Cobb 500 and Ross 308), while it may require individual stage- and organ-specific adaptations for other, e.g., layer chicken, breeds.

\subsection{Cell culture}

MDA-MB-231 (ECACC 92020424) cells were expanded by culture in complete culture medium (CCM) prepared from Dulbecco's Modified Eagle's Medium (DMEM/F12/Ham medium, \#D8437, Sigma- Aldrich) supplemented with 10\% fetal bovine serum (FBS, \#12003C, Sigma Aldrich) and 1\% Penicillin-Streptomycin (PS, 10,000 U/mL; \#15140122, Gibco) under standard conditions $\left(37^{\circ} \mathrm{C}\right.$, humidified, $5 \% \mathrm{CO}_{2}$ gas atmosphere). Culture medium was changed every two days and the cellular growth was controlled by using a phase-contrast microscope and cell counting. According to the cell counting data, the average population doubling time of MDA-MB-231 cells (used at the experiments in the passages 4 to 6 ) was approximately $34 \mathrm{~h}$, with the average viability $\sim 98 \%$. The same culture medium was used for all in vitro experiments, unless otherwise specified.

\subsection{Recellularization of CE acellular organ-sepcific scaffolds (AOSS) with MDA-MB- 231 cells}

Small fragments (approximately, $2 \times 3 \mathrm{~mm}$ ) of decellularized CE organs/tissues were cut by a scalpel and put into 24-well flat bottom tissue culture plates (Costar, Corning, $\# 3524$ ). One $\mathrm{mL}$ of $0.1 \%$ peracetic acid solution (\#77240, Sigma-Aldrich) in $4 \%$ ethanol was added to every well for $2 \mathrm{~h}$. Then this solution was removed and the AOSSes were washed with sterile PBS (0.4 mL per well) twice and sterilized by ultraviolet light in a tissue culture hood for $45 \mathrm{~min}$ (with turning the scaffolds with sterile tweezer twice to expose different sides). Next, each well was refilled with $1 \mathrm{~mL}$ of CCM. Following that, the plates with the AOSSes were placed into a tissue culture incubator and conditioned overnight under humidified atmosphere with $5 \% \mathrm{CO}_{2}$ at $37^{\circ} \mathrm{C}$. This step served for the scaffolds' conditioning and for an additional check of sterility. 
MDA-MB-231 cells $\left(1 \times 10^{5}\right.$ cells in a $20-\mu \mathrm{L}$ drop of CCM $)$ were seeded on the top of the AOSS obtained by iWO-DCL of the CE the livers, lungs, hearts, ventriculi and proventriculi, brains, small intestines, and breast muscles to form 3D TECs. One TEC was placed per a well of 24-well culture plate. Control scaffolds were left unseeded. Next, the cells were allowed to attach to the substrates for $2 \mathrm{~h}$ in a tissue culture incubator, then added with $1 \mathrm{~mL}$ of complete culture media per well and cultured for 1-28 days. On day 1, the TECs were carefully relocated to the new multiwell plates to preserve only the cell populations that attached to the scaffolds. The CCM in the growing 3D cultures was carefully changed twice a week. For microscopy, viability assays and histological analysis, TECs were sampled on week 1 (the multiorgan panel of TECs based on CE the livers, lungs, hearts, ventriculi and proventriculi, brains, small intestines, and breast muscles) or weeks 1, 2, 3 and 4 after seeding (liver-based TECs).

\subsection{Analysis of structural evolution of TECs}

\subsubsection{Histology, histomorphometry and fluorescence microscopy}

AOSSes and TECs were fixed in 10\% neutral buffered formalin, dehydrated in a graded series of alcohols, embedded in paraffin wax, and cut into serial sections of $5 \mu \mathrm{m}$ in thickness by a rotary microtome. After deparaffination, slices were stained with hematoxylin and eosin (H\&E), Van Gieson's picrofuchsin, Masson's trichrome and toluidine blue, following conventional protocols. Stained histological preparations were examined using an upright research microscope Axio Imager Z2 (Zeiss, Germany) equipped with dry-air EC Plan-Neofluar (5×/NA0.16; 10×/NA0.30; 20×/NA0.50 Ph) and an oil-immersion Plan Apochromat (100×/NA1.46 oil) objectives (Zeiss, Germany). Relative cellularity of the TECs was examined using ImageJ software via color intensity-based segmentation of the area occupied by cells relative to the total section area of the TEC on the images of H\&E stained samples. The images were recorded using a preinstalled microscope digital video camera AxioCam (1388×1040, Zeiss, Germany) in a single-frame and stitching modes using Zen 2012 software. For the rapid check of the quality of DCL and the viability of the cells in TECs, epifluorescence microscopy was performed. For the DCL quality check, the deparaffinized section of AOSS were stained with DAPI (Sigma-Aldrich, \#D9542) to detect nucleic acids and with Phalloidin-TRITC (Sigma-Aldrich, \#P1951) to detect f-actin, following the manufacturer's protocol. For the live-dead cell detection, the TECs were stained immediately with fluorescein diacetate (FDA; \#F7378, Sigma-Aldrich) and propidium iodide (PI; \#P4864, Sigma-Aldrich) according to the Application Note \#33 by Ibidi GmbH (Germany) [46], counterstained with DAPI and imaged with the use of the filter settings for DAPI, and FITC (for FDA) and PI on the same microscope.

\subsubsection{Scanning electron microscopy}

The samples of AOSSes and TECs were fixed in 2.5\% buffered glutaric aldehyde, further dehydrated in $70-100 \%$ alcohols and contrasted by $\mathrm{OsO}_{4}$ using the Pelco Biowave Pro+ microwave processing system (PELCO BioWave Pro, Ted Pella Inc., USA) and underwent critical point drying in Tousimis Autosamdri-815 Critical Point Dryer (Tousimis research corporation, USA). Afterwards, the samples were mounted on stabs with conductive carbon/graphite paint (ProSciTech, Australia) and coated with platinum using an Emitech K575x Pt sputter coater (Emitech Ltd., UK). Electron microscope images were taken using the NanoSEM 230 is a field-emission scanning electron microscope (FE-SEM) under accelerating voltage $5 \mathrm{kV}$, work distance $20 \mathrm{~mm}$ and size point 30 by the secondary electron imaging mode.

\subsubsection{Atomic force microscopy}

AFM imaging of the deparaffinized/ non-coverslipped dry histological tissue (CE liver AOSS) sections on glass slides was performed in air in the semi-contact mode (according to the method established by us earlier [47] with modifications indicated below). The scans were taken following the selection of the regions of interest under bright field 
microscopy. The following regions of interest were explored in the CE liver AOSSes: the parenchymal ECM of former Disse's space, the former parenchymal ECM in the vicinity of vein and arterial walls, as wells as the former walls of the arteries, veins and venules.

The AFM measurements were performed on the Bruker Dimension ICON SPM. The DMT modulus were measured using peak force tapping mode with the OTESPA-R3 probe (Bruker AFM probes). For accurate modulus measurements, the probe was calibrated according to the following procedure. Firstly, the probe was withdrawn from the sample surface by at least $5 \mathrm{mms}$, so the spring constant could be calculated using the thermal tunning method. Then, the probe is engaged on a hard surface, namely, sapphire, with a engage set point of $0.5 \mathrm{~V}$, and then the probe is ramp on the surface with $0.2 \mathrm{~V}$ of deflection to calculate the deflection sensitivity, which was determined to be $67.3 \mathrm{~nm} / \mathrm{V}$ (an average of 5 measurements are taken per location and two locations were measured). After the ramp curves are done, the probe is calibrated again and then the spring constant is updated to a more accurate value. In this case, $28 \mathrm{~N} / \mathrm{m}$.

The tip radius of the probe was checked with a titanium roughness check, this is a sample with sharp titanium flakes to check the tip radius, a slow scan rate of $0.2 \mathrm{~Hz}$ was used, as the features of the titanium sample were sharp and rough, which could damage the probe apex if the scan rate was too fast. After all the constants are determined, the probe was engaged on the tissue sample, and the scanning parameters, such as scan rate, peakforce setpoint, feedback gain were optimized accordingly depending on the scan size and the scanning area. The resolution of image was kept at 256 samples/line. The following is an example of the typical scan parameters that were used: Scan size $5 \mu \mathrm{m} \times 5 \mu \mathrm{m}$; Scan rate 0.25 to $0.4 \mathrm{~Hz}$; Feedback gain $\sim 14$; Peakforce set point $70 \mathrm{nN}$, and Poisson ratio 0.45 . The data visualization and analysis of stiffness and surface roughness were performed using free Gwyddion 2.55 Software (Czech Metrology Institute, Czech Republic). The statistical calculations were done using SPSS 26.0 as described below.

\subsubsection{Image analysis for histological morphometry}

Image processing techniques were applied to evaluate the cellular distribution and shapes in H\&E stained histological images of the evolving CE liver\& human TNBC TECs acquired on Weeks 1, 2, 3, and 4 of in vitro culture. During pre-processing, Gaussian blur with s.d. $\sigma=0.65 \mu \mathrm{m}$ was applied to reduce high-frequency components. Using color deconvolution, the images were split in three separate channels containing cells, matrix, and background respectively. Next, images with cells were thresholded and segmentation algorithm was performed where possible to extract single cells boundaries. Cell shape was described by calculating the circularity of a convex hull. The reason for using convex hull approximation of cell boundaries is that it reduces variability of the data and allows more accurate classification. The boundaries of the parenchymal and stromal compartments were outlined manually. Image processing was performed using ImageJ and MatLab 2016b.

2.5.5. MTT assay for evaluation of the population evolution of MDA-MB-231 cells in 2D and 3D TECs

The cell viability was tested in 3D liver-TNBC TECs and in matching $2 \mathrm{D}$ cell cultures of MDA-MB-231 cells using a modified MTT colorimetric assay. This assay relies on the reduction and conversion of yellow 3-(4,5-dimethylthiazol-2-yl)-2,5-diphenyltetrazoliumbromide (MTT) reagent (\#M2128, Sigma-Aldrich) into purple formazan salt, where the optical absorbance of formazan crystals dissolved in dimethyl sulfoxide (DMSO) represents the activity measure of mitochondrial dehydrogenase[48]. The tested cultures were grown in complete culture medium in a humidified atmosphere under $5 \% \mathrm{CO} 2$ at $37^{\circ} \mathrm{C}$.

The following procedure was introduced to ensure appropriate comparison between 2D and 3D TEC cultures. The $5^{\text {th }}$ passage MDA-MB-231 cells were seeded on chick embryo liver AOSSes, as described earlier, while the same amount of the cells $\left(1 \times 10^{5}\right.$ in a $30-\mu \mathrm{L}$ drop of complete culture media) was deposited in the middle of 24 -well culture plate 
(Costar) wells to perform high-density seeding. Next, cells in both cultures were allowed to attach to the substrates for $2 \mathrm{~h}$ in a tissue culture incubator in a humidified atmosphere under $5 \% \mathrm{CO}_{2}$ at $37^{\circ} \mathrm{C}$, and then filled with $1 \mathrm{~mL}$ of complete culture media per well and cultured for 1 day.

After $24 \mathrm{~h}$, the media was removed, and the samples were washed twice with PBS to eliminate unattached cells. Next, 3D TECs were aseptically transferred to new 24-well culture plates to get rid of the cells adhered to the plastic and not to the scaffolds in the original cultures, then filled with fresh complete culture media $(1 \mathrm{~mL}$ per well) and cultured for 4 weeks as described above. At the same time, 2D cell cultures after washing with PBS, were filled with complete culture media, and cultured for 4 weeks, without splitting. The media was changed twice a week in both types of cultures.

The MTT assays were carried out on days 1, 7, 14, 21 and 28 after seeding (day 1 and Weeks 1, 2, 3 and 4, respectively). For each assay, 3 samples of TECs were randomly selected and transferred to a separate 24-well plate for testing, while 3 wells of cells growing in 2D culture were used as the internal control. After double washing with PBS, $500 \mu \mathrm{L}$ of MTT reagent $(0.5 \mathrm{mg} / \mathrm{mL}$ in the phenol red free cell culture medium; DMEM/F12; \#D6434, Sigma Aldrich), was added to each well. Then the samples were incubated at $37^{\circ} \mathrm{C}$ in a tissue culture incubator for $4 \mathrm{~h}$ to allow precipitation of insoluble formazan crystals. After that, the supernatant was carefully collected and $500 \mu \mathrm{L}$ of DMSO was added to the wells and left for $15 \mathrm{~min}$ in the dark on a rocking platform at room temperature to dissolve purple formazan crystals. Next, four portions of $100 \mu \mathrm{L}$ of the dissolved MTT product was taken from each well, transferred to separate wells of a clear 96-well culture plate (\#3585, Costar, Corning) and used for absorbance measurements. The samples' absorbance was measured in a spectral band centered at $570 \mathrm{~nm}$ by a PHERAstar multiplate reader (BMG Labtech, Germany), with unseeded wells used as blank controls. The experiments were repeated twice, with at least duplicates for each condition; the results were corrected for the blank controls by MARS Data Analysis software (BMG Labtech, Germany).

\subsubsection{Modelling cell growth dynamics}

The logistic growth model was fitted to the cell viability values at each point in time in $2 \mathrm{D}$ and $3 \mathrm{D}$ cultures (Equation 1 ):

$$
N=\frac{C_{0} C_{\max }}{C_{0}+\left(C_{\max }-C_{0}\right) e^{-d t}}
$$

where $N$ is proportional to the number of viable cells at time $\mathrm{t}_{\text {; }} C_{0}$ is proportional to the number of viable cells at the start; $C_{\max }$ is proportional to the maximum number of viable cells, and $d$ is the growth rate. The fit was performed using nonlinear least squares function in Matlab software.

\subsection{Evaluation of angiogenic potential of liver-TNBC TECs in vivo (CAM assay)}

\subsubsection{Grafting and imaging procedures}

The detailed description of the used procedures of angiogenic assay on chick embryo CAM [49] can be found in Appendix A, Section A 2.4 and Figures A11 and A12. Briefly, liver-TNBC TECs, CE liver AOSSes or MDA-MB-231 cell suspensions were grafted on CAM separately, one sample of each material type per egg, 5 replicates per a group. Before grafting, the TECs were cultured in vitro for 12 days as described above, reaching the stage when they contained approximately $2 \times 10^{5}$ viable cells (according to MTT assay data). The liver AOSSes were kept in complete culture media for $24 \mathrm{~h}$ before grafting on CAM. The suspension of $2 \times 10^{5} \mathrm{MDA}-\mathrm{MB}-231$ cells in $60 \mu \mathrm{L}$ of CCM were grafted on CAMs within a sterile rubber ring. The angiogenic effect induced by the TECs, liver AOSSes and suspensions of MDA-MB-231 cells in PBS grafted on CAM was evaluated by stereomicroscopy imaging performed on the day of grafting (embryonic day 8, ED8) and on ED12, in comparison to the natural growth of blood vessels of CAM occurring during the same period of chick embryo development. 


\subsubsection{Angiogenesis quantification}

The images of CAM taken using $2 \times / 0.5$ N.A. objective were processed using the following methodologies to evaluate the dynamics of the vascular length density and branching of blood vessels in CAMs. For Control and Cells groups, 10 regions of interest (ROIs) per egg were chosen manually. CAMs grafted with 3D engineered tumors (TECs) and liver AOSS (Scaffolds) were imaged from the 4 corners around the graft, and then 34 ROIs were chosen from every corner to obtain approximately 10 ROIs per egg. Since the vessels have higher contrast in the green channel, the RGB images were split to obtain the green component. Histogram stretching of grey level intensities was performed to enhance the image contrast. Then, a ridge detection algorithm [50] involving convolution with the derivatives of a Gaussian smoothing kernel was used to capture the blood vessels by finding local minima, resulting in a skeleton of a vascular pattern. For each ROI, the total branch length of the obtained skeleton was divided by the area of the ROI and then the results were averaged to get the mean vascular density per egg. The exploration of the branching parameters of the vascular trees was performed using the Angiogenesis Analyzer plugin for ImageJ [51] (see Appendix A, Section A2.4.3 for the details). Image processing was performed using ImageJ and MatLab 2016b.

2.7. Cytotoxicity and cellular uptake of doxorubicin (Dox) and mesoporous silica nanoparticles
loaded with Dox in $3 D$ liver-TNBC TECs and in matching 2D cultures of TNBC cells

2.7.1. Preparation of mesoporous silica nanoparticles and their loading with Dox

Anionic surfactant-templated mesoporous silica nanoparticles (AMS-6) that have been reported by us earlier [52,53] were synthesized in-house following a protocol described elsewhere [53]. Briefly, N-lauroyl-L-alanine was used as surfactant, APES was applied as a costructure directing agent to achieve connected pores in TEOS-sourced silica nanomaterial. The sample was calcinated at $\sim 550^{\circ} \mathrm{C}$ using the temperature gradient of $1.5^{\circ} \mathrm{C} / \mathrm{min}$ to remove surfactant. Next, as-synthesized mesoporous silica nanoparticles (AMS-6) were loaded with 20\% Dox (Doxorubicin hydrochloride, \#D1515, Sigma-Aldrich). Dox diluted in 100\% ethanol was added to AMS-6 nanoparticles in a round bottom flask mounted on a rotary evaporator, and ethanol was evaporated at $40^{\circ} \mathrm{C}$ under vacuum with slow rotation. The collected sample was air dried overnight.

\subsubsection{Characterization of mesoporous silica nanoparticles}

Unloaded ("pure") AMS-6 and Dox-loaded AMS-6 (AMS-6-Dox) were characterized using transmission electron microscopy (TEM), X-ray diffraction (XRD), thermogravimetric analysis (TGA) and dynamic light scattering (DLS). Nitrogen adsorption/desorption isotherm measurements were carried out to evaluate the effective surface area of the AMS6 and AMS6-Dox samples. For TEM sample preparation, a small amount of dry AMS-6 or AMS-6-Dox was thoroughly crushed in a mortar and then diluted in ethanol. A drop of the suspension was placed on a copper grid and dried. Next, the grid was placed in a sample holder of a JEOL 3000F TEM (Peabody, USA) and imaged at $300 \mathrm{kV}$ with the resolution of 1.6 A. Images were obtained using Gatan SC1000 11-megapixel CCD camera (Pleasanton, USA), with a $1024 \times 1024$ pixel Gatan image filter (Pleasanton, USA). XRD measurements of $20 \mathrm{mg}$ of the dried nanoparticles' samples were carried out using XRD instrument Bruker D8 Discover equipped with VÅNTEC-500 detector featuring a $140 \mathrm{~mm}$ diameter window (Billerica, USA).

XRD patterns were recorded using $\mathrm{Cu} \mathrm{K} \alpha$ anode $(\lambda=0.1542 \mathrm{~nm})$, operating at $40 \mathrm{kV}$ and $30 \mathrm{~mA}$. TGA measurements were performed using 1-mg sample placed in an aluminum crucible (TA Instruments TGA2050, New Castle, USA) and heated from 25 to $850^{\circ} \mathrm{C}$ at $10^{\circ} \mathrm{C} / \mathrm{min}$ under air flow at $10 \mathrm{~mL} / \mathrm{min}$. Nitrogen adsorption/desorption isotherms were acquired at a temperature of $-196^{\circ} \mathrm{C}$ using liquid nitrogen with a TriStar II by Micromeritics ${ }^{\circledR}$ instrument (Norcross, USA), following the AMS-6 and AMS-6-Dox samples degassing under vacuum using VacPrep ${ }^{\mathrm{TM}} 061$ by Micromeritics ${ }^{\circledR}$ instrument (Norcross, USA) 
for $\sim 10 \mathrm{~h}$ at $120^{\circ} \mathrm{C}$. The surface area was calculated using the Brunauer-Emmett-Teller (BET) equation [54].

Hydrodynamic diameters and the zeta-potentials of colloidal AMS-6 and AMS-6Dox were measured in PBS and complete culture media $(1 \mathrm{mg} / \mathrm{mL})$ by Zetasizer Nano ZS (Malvern, UK) in three runs followed by averaging.

2.7.3. MTT viability assay of 2D and 3D TEC cell cultures treated with free and nanoformulated Dox

The TECs were cultured for 3 weeks as described above. For control 2D in vitro culture MDA-MB-231 cells were seeded in 96-well plates (Costar, Corning, \#3599) at the density of $2 \times 10^{4}$ cells per well and incubated in complete culture medium for $24 \mathrm{~h}$ before the test. Then culture medium was removed from all the cultures, TECs were aseptically transferred to new 24-well culture plates and all the cultures were washed 3 times with PBS. Free Dox of the concentrations ranging from 0.1 to $10 \mu \mathrm{g} / \mathrm{mL}$, AMS-6 $(50 \mu \mathrm{g} / \mathrm{mL})$ and AMS-6-Dox of the concentrations ranging from 0.5 to $50 \mu \mathrm{g} / \mathrm{mL}$ (Dox-equivalent, $0.1-10$ $\mu \mathrm{g} / \mathrm{mL}$ ) were diluted in complete culture media and sonicated immediately before the test. Each concentration of each type of the tested compounds was applied in a total volume of $100 \mu \mathrm{L}$ to 8 parallel wells of 96-well plates for challenging of 2D culture of MDAMB-231 cells. At the same time, 2 parallel TECs growing in 24-well plates were used for testing of each concentration of each tested compound, and the added volume of the dispersions was $400 \mu \mathrm{L}$ per a well. The 2D and 3D cultures treated with complete culture medium were used as a control. The exposure time was $36 \mathrm{~h}$. Then MTT tests were performed, as described above (see Section 2.5.5). Then the supernatant was removed and $100 \mu \mathrm{l}$ or $400 \mu \mathrm{L}$ of DMSO was added to the wells of 96-well plates (2D cultures) and 24well plates (3D TECs), respectively. The solution of formazan in DMSO from the TECs was transferred to the new 96-well plate ( $100 \mu \mathrm{L}$ per a well; 3 samples per a TEC), and the absorbance of the tested cultures was measured. The mean percentage of dead cells $E$ and the standard deviation $\sigma E$ were recalculated based on the absorbance of the controls using the Equation (2):

$$
E=\left(1-\frac{A}{A_{0}}\right) \times 100 \%, \quad \sigma_{E}=\left|\frac{100}{A_{0}}\right| \sigma_{A},
$$

where $\mathrm{A}$ is the average absorbance in each group, $\mathrm{A}_{0}$ is the average absorbance of the corresponding control, $\sigma \mathrm{A}$ is the standard deviation of the absorbance in each group.

\subsubsection{Pharmacodynamics modelling}

The experimental measurements of the percentage of dead cells in 2D and 3D cultures after administration of free or nanoformulated Dox were described by the following sigmoid Equation 3:

$$
E=\frac{E_{\text {max }}}{1+10^{\frac{E c 50-\text { dose }}{h}}}
$$

where Emax is the maximum effect (\%), EC50 is the half maximal effective concentration $(\mu \mathrm{g} / \mathrm{mL})$, dose is the concentration of administered Dox $(\mu \mathrm{g} / \mathrm{mL})$, and $\mathrm{h}$ is the Hill coefficient. The half maximal inhibitory concentration (IC50), i.e. the drug concentration needed to obtain $50 \%$ of cell death, was calculated in each group using the line of the best fit. The parameters of the model are provided in Tables A6 and A7, respectively, for free and nanoformulated Dox (Appendix A).

The fit was performed using a weighted unconstrained nonlinear curve fit (Matlab r2016b) with the inverse error as the weight, and the dose values were first logarithmically transformed. The $0 \%$ cell death ( $100 \%$ viability) at $0 \mu \mathrm{g} / \mathrm{mL}$ in controls was given a higher weight as the data was normalized to these values and the model is expected to approach this intercept closely. 
2.7.5. Confocal microscopy study of the uptake of free and nanoformulated Dox in 3D TEC and 2D cultures

As produced liver-TNBC TECs were cultured for 3 weeks. Control 2D in vitro cultures of MDA-MB-231 cells were seeded onto sterile coverslips placed into wells of a 24well plate (Costar) at the density of $5 \times 10^{4}$ cells/well and incubated under standard conditions in $1 \mathrm{~mL}$ of complete culture media during $24 \mathrm{~h}$ prior to the observation. Dox solution $(10 \mu \mathrm{g} / \mathrm{mL})$, AMS-6 nanoparticles $(50 \mu \mathrm{g} / \mathrm{mL})$, and AMS-6-Dox nanoparticles $(50 \mu \mathrm{g} / \mathrm{mL}$; Dox-equivalent, $10 \mu \mathrm{g} / \mathrm{mL}$ ) dispersions were prepared as described above. Next, after removal of the culture media and triple washing with PBS the tested compounds were added to the wells with 2D and 3D cultures in a total single volume of $0.5 \mathrm{~mL}$ per well. The wells added with CCM without Dox or nanoparticles were used as controls. The prepared $2 \mathrm{D}$ and $3 \mathrm{D}$ cultures were incubated for $24 \mathrm{~h}$ in tissue culture incubator at $37^{\circ} \mathrm{C}$ an $5 \% \mathrm{CO}_{2}$, and, next, following 3 times thorough rinsing with PBS to remove free Dox and nanoparticles, they were fixed with $10 \%$ neutral buffered formalin at room temperature.

After 24-h fixation process and another washing with PBS, the samples were stained with DAPI solution in PBS (\#D9542, Sigma-Aldrich) for $20 \mathrm{~min}$ at $37^{\circ} \mathrm{C}$. Next, the staining solution was removed, the coverslips were washed twice with PBS to eliminate unbound DAPI. Finally, the samples were mounted on glass slides with Dako anti-fade mounting media and sealed with nail polish.

Dox and AMS-6-Dox cellular uptake was imaged by an inverted Zeiss LSM 880 laser scanning confocal microscope (Zeiss, Germany), using a Plan Apochromat 10×/0.45 N.A. M27 and Plan Apochromat 40×/1.3 N.A. oil DIC UV-IR M27 objectives. Dox fluorescence was observed using $488 \mathrm{~nm}$ excitation and emission 535-673 nm; DAPI fluorescence was observed using 405-nm excitation and emission 411-528 nm.

\subsection{Statistical analysis}

The data were expressed as means \pm standard deviations (SD), and the $95 \%$ confidence intervals ( $\mathrm{CI}_{95 \%}$ ) for the means were calculated. Due to the non-Gaussian nature of the data the two-sided Mann-Whitney $U$ test was used to evaluate the significance of intergroup differences between the means. The two-sampled Kolmogorov-Smirnov test was used to compare any two observed distributions of branch length density. Statistical significance was reported as follows: ${ }^{*} p<0.05,{ }^{* *} p<0.01,{ }^{* * *} p<0.001$ or the exact $p$-value was provided where possible. Statistical analyses were performed using R Statistical and SPSS 26.0 Software.

\section{Results}

3.1. Soils and seeds: development of a CE-based multiorgan tissue engineering platofrm for modelling of cancer micrometastases

The iWO-DCL induced significant macroscopic changes of organs emerged as gradual loss of native color towards whiter and more translucent appearance, and the volume reduction (Figure A2 in Appendix A). The time of decellularization varied depending on the organs' original volume and density (See Table A1 in Appendix A). In originally nontranslucent organs, it was notable that during iWO-DCL the discoloration develops centripetally: it started from the edges of the tissue and then spread to the rest the organ in a patchy way and later converted into diffuse whitish color and semitransparency. The majority of decellularized organs, excepting brain, preserved the shape reflecting to the original one, while the matrix became loosened to a certain extent.

Histological examination confirmed complete removal of the cellular material by iWO-DCL in all studied types of CE organs The ECM generally preserved its organ-specific architecture (Figure 2). All obtained types of CE AOSS were successfully recellularized with human MDA-MB-231 cells and formed viable 3D TECs indicating the biocompatibility of AOSS, the efficient cellular adhesion and the notable proliferation on the matrices as was observed for at least 1 week. In the longitudinal experiments, the TECs combining several types of CE AOSS and TNBC cells were confirmed to be able to successfully 
survive for at least 2-3 weeks in vitro (data not shown), indicating the potential for the detailed studies on the interactions of the organ-specific ECMs and cancer cells. Importantly, MDA-MB-231 cells demonstrated organ-specific patterns of colonization of the ECM originating from different organs (Figure 2 a).

The structure of ECM in AOSS was changing during the colonization by TNBC cells. In particular, the visual homogeneity of the matrices was becoming visible. This was notable starting the first week of in vitro culture but progressed at the later stages of the TECs development.

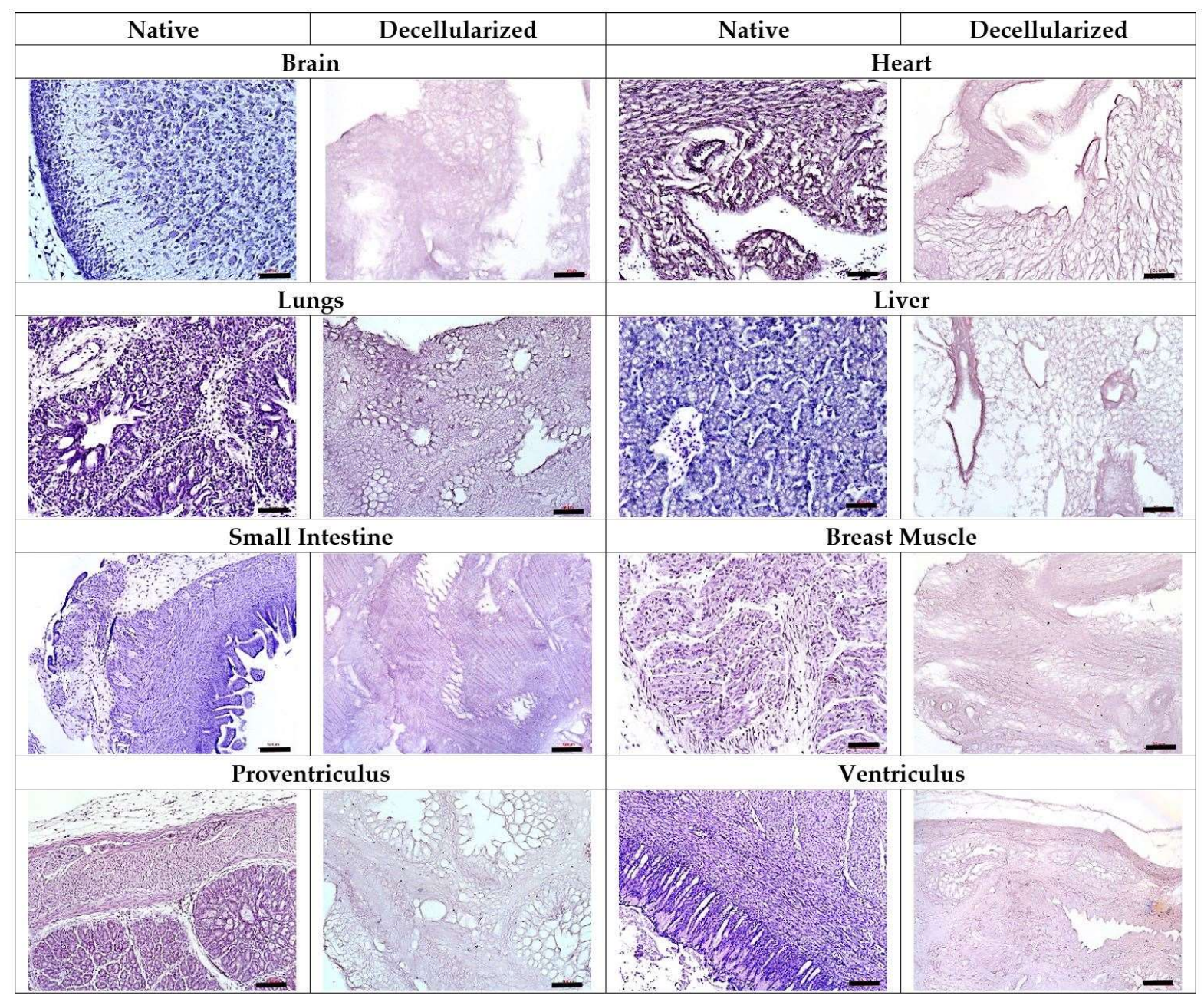

Figure 2. Effect of iWO-DCL on various organs of ED18 CE. Note complete removal of the cells. The specialized compartments of the organs are recognizable in the matching AOSSes, excepting the brain AOSS, where only ECM areas of different density, with more fibrous (on the left) or more sponge-like (on the right) structures can be seen. In the heart AOSS, the myocardium and endocardium compartments are discernible. In the lungs AOSS, the specialized avian pulmonary architecture is preserved with clearly visible ECM of parabronchi, alveolar and air capillaries and the interparabronchial septae. In the liver AOSS, the elements of former parenchyma (mostly Disse's space ECM) and stroma (central and elements of triads) are present. In the small intestine AOSS, the histoanatomically preserved ECM all the conventional tissue layers of the organ is visible. The breast muscle AOSS contains the interchanging areas of the denser and aligned and more loose ECM elements corresponding to the distribution of the muscular bands as well as the ECM of arteries and veins. The AOSSes obtained from the proventriculus and ventriculus demonstrate excellent preservation of the histoanatomy of the organ layers and glandular elements. Staining with Hematoxylin and Eosin. Scale bars: 50 um (Brain, Heart, and Lungs, Liver, and Breast Muscle); and $100 \mu \mathrm{m}$ (Small Intestine, Proventriculus, and Ventriculus).

Figures $2 \mathrm{~b}$ and $2 \mathrm{c}$ illustrate the results of the "soils and seeds" test showing the effect of organ-specific ECMs on the relative amount of cells able to attach and grow on these substrates. The highest relative cellularity was observed in TECs formed by CE lungs 
AOSS and MDA-MB-231 cells. This was followed by the TECs containing TNBC cells and the CE AOSS derived from the ventriculi, breast muscles, small intestines, liver, proventriculi, brains and hearts. At the level of statistical significance, the lungs ECM supported higher cellularity than the ECM of proventriculi, brain and heart matrices; the ECM derived from ventriculi was more cellularized than the ECMs of brain and heart, and small intestine matrix maintained higher cell density than the AOSS prepared from the CE brains and hearts. Also, statistically, equally efficient colonization by TNBC cells was observed in the AOSS obtained by iWO-DCL of CE lungs, ventriculi, breast muscles, small intestines, liver and proventriculi, indicating statistically equal risk of the initiation of the TNBC micrometastases that could be attributed to the role of the organ-specific ECM, with a few exceptions. In particular, our results show that the ECM of lungs may stronger contribute to the increased risk of the secondary tumors than the brain ECM, while the liver ECM has an intermediate position between these two organs (see Figure $2 \mathrm{c}$ ).

In the next stage of the study, we concentrated on the interactions between the liverspecific ECM (LS-ECM) and TNBC cells in 3D TECs and on applications of this tissue engineering model in cancer research.
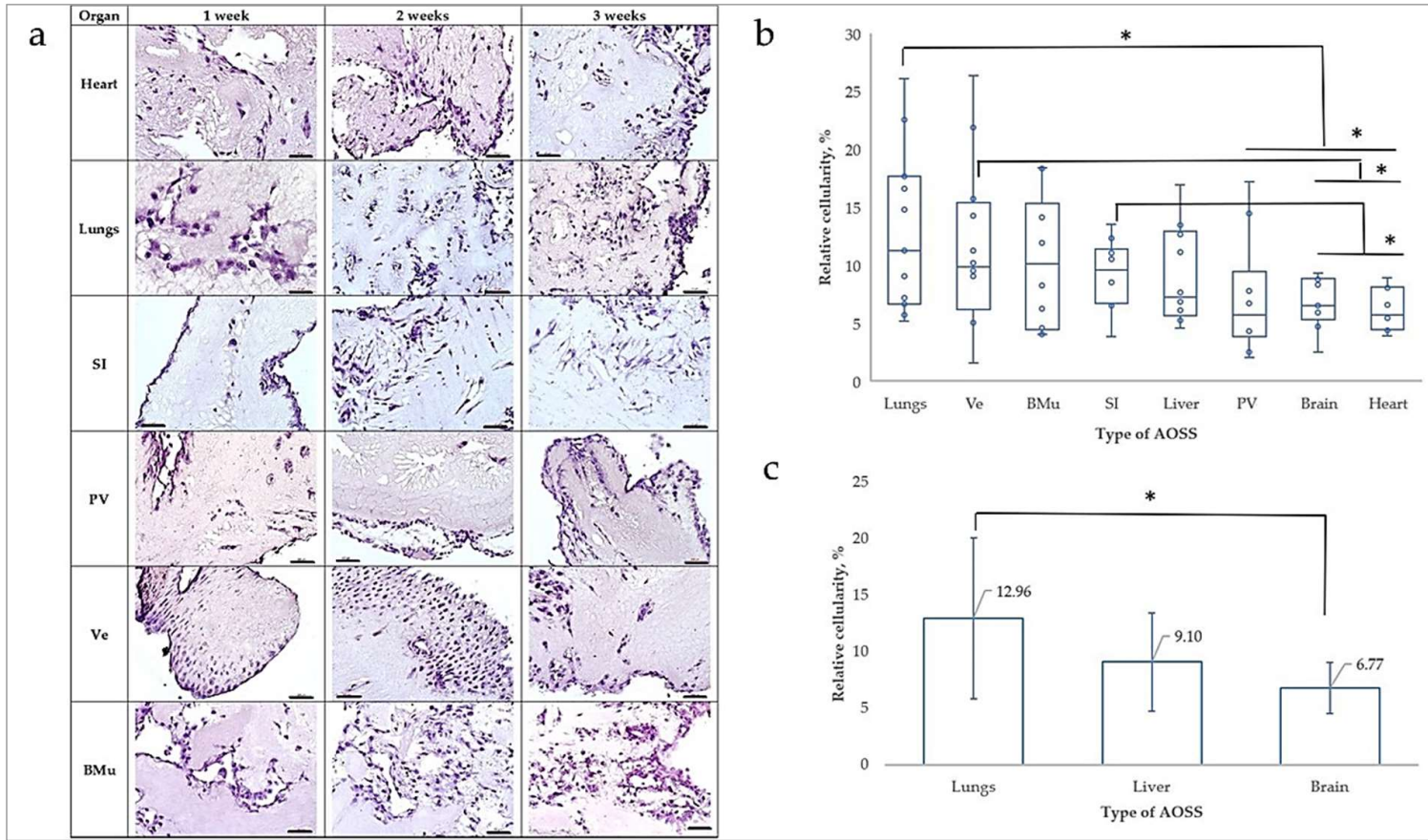

C

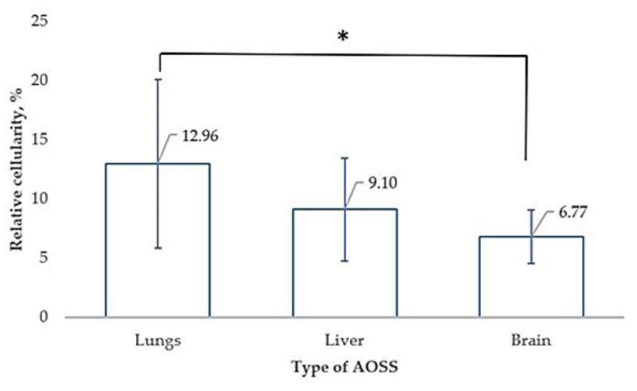

Figure 3. (a) Structural evolution of the organ-specific TECs that combine human MDA-MB-231 cells and CE AOSSes as observed histologically during 3 weeks of in vitro culture. All the TECs demonstrate initial colonization of the surface areas with cancer cells followed by the progressive invasion into the deeper parts of the scaffolds with increasing total cellularity. Note the organ-specific colonization patterns such as cells' growth in association with the luminal ECM elements such as the former walls of blood vessels in the heart and $\mathrm{BMu}$, and colonization of parabronchial ECM in the lung TECs; linear pattern ("Indian-files") of tumor invasion along the former muscular layers in SI ECM and the tunica muscularis in Ve; and the templated colonization of the former glandular elements and secretory lining of PV and Ve. Staining with Hematoxylin and Eosin. Scale bars: $50 \mu \mathrm{m}$ (Brain, Heart, Lungs, SI, and BMu); and $100 \mu \mathrm{m}$ (PV and Ve). (b) Relative cellularity of the TECs combining MDA-MB-231 cells and AOSSes obtained from the different organs of origin on the $7^{\text {th }}$ day of in vitro culture. Blue circles indicate the individual values, the blue horizontal lines show the Mean, the boxes represent the range between $25^{\text {th }}$ and $75^{\text {th }}$ percentiles and the whiskers reflect the minimal and maximal observed values. Statistically significant differences $\left(\mathrm{p}<0.05,{ }^{*}\right)$ between the studied TECs are shown by black lines. (c) Relative cellularity (Mean \pm Standard Deviation) of the MDA-MB-231/AOSS TECs representing the common cites of the distant organ metastases of breast cancer. Statistically significant difference $\left(\mathrm{p}<0.05,{ }^{*}\right)$ between the lung and brain TECs is shown by a black line. Abbreviations: SI - Small Intestine, PV - Proventriculus, Ve - Ventriculus, BMu - Breast muscle. 


\subsection{1. iWO-DCL reveals compartmental organization of LS-ECM}

The AOSS were successfully prepared from the livers of CE. The native structure of ED18 CE liver and the effects of applied iWO-DCL procedure are detailed in Sections A2.1 and A2.2s and illustrated by Figures A3, A4 and A5 in the Appendix A. Briefly, iWO-DCL efficiently removed cellular elements, while the liver ECM was well preserved (Figure 4) and structurally similar to the decellularized liver of other vertebrates [55-57].

DCL revealed two histoanatomical compartments in the LS-ECM that randomly appeared in AOSS (see Figure 2 a and b). We term the first compartment as "parenchymal" (see Figure $4 \mathrm{c}$ ) and the second as "stromal" (see Figure $4 \mathrm{~d}$ ), for simplicity. The ECM composition of these compartments was relatively similar to each other, while the architecture differed significantly. The first compartment contained sponge-like structures corresponding to the ECM of the former hepatic parenchyma and formed mainly by the residuals of the Disse's space elements. The second compartment was formed by denser structures relatively enriched with fibrillar collagen (see Figure $4 \mathrm{~b}$ ) mostly corresponding to the former stromal elements such as walls of central veins, portal triads, and interlobular connective tissue sheaths and capsular connective tissue (see Figures A4 and A5 in Appendix A).

The relative area of the parenchymal and stromal compartments on the histological sections of LS-ECM (in the CE liver AOSS) was approximately equal ( $\approx 48 \pm 21 \%$ and $52 \pm$ $17 \%$, respectively).
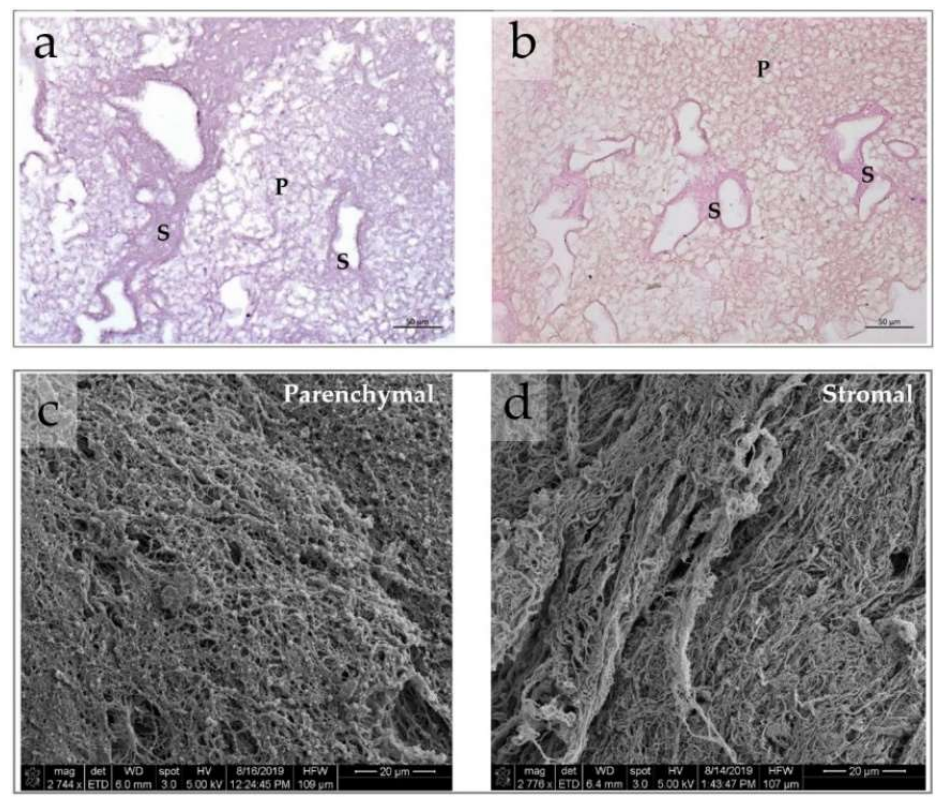

Figure 4. $(\mathbf{a}, \mathbf{b})$ Histological features of CE liver AOSS. Complete removal of cells and compartmental structure of the liver ECM are clearly visible in samples stained by hematoxylin and eosin (a) and by Van Gieson's (VG) (b) methods. The elements of the parenchymal (labelled " $\mathrm{P}$ ") and stromal (labelled "S") compartments of the CE liver ECM. The ECM of parenchymal compartment is loose and mesh-like, with randomly oriented matrix elements of the former Disse's space; while the ECM of the stromal compartment is denser, with more aligned and more fuchsinofilic (pinkred when stained by VG method). The VG staining reveals the collagenous nature of the liver AOSS and indicates the higher contents of fibrous/crosslinked collagen in the stromal compartment in comparison to the parenchymal one. Scale bars: $50 \mu \mathrm{m}$. (c, d) SEM images of CE liver AOSS. The fragments representing parenchymal (c) and stromal (d) compartments are shown. Parenchymal compartment is characterized by fine, random, mesh-like structure, while the stromal LS-ECM is composed by coarser and more parallel aligned fibrillar elements.

The compartments of the LS-ECM were further examined using AFM (Figure 5 and Figures A6, A7 and A8 in Appendix A). The AFM analysis of the AOSS in the hydrated state indicated that the Young's Modulus of the LS-ECM equals approximately $0.2 \mathrm{kPa}$ 
(See Figure A6 in Appendix A). However, it was not possible to measure the mechanical characteristics of the scaffolds in wet form due to the very prominent surface topography. Therefore, we applied the AFM analysis of the dehydrated sections of the AOSS by the method proposed by us elsewhere [47].

Interestingly, the surface topography (see Figure $5 \mathrm{a}$ and c) and mechanical properties (see Figure $5 \mathrm{~b}$ and $\mathrm{d}$ ) of the dehydrated matrices of parenchymal and stromal origin varied depending on the scale of the measurements. The surfaces of the ECM derived from the CE liver parenchyma were highly uneven, in comparison with the more flattened stromal ECM. This feature was reflected by statistically significant difference in the surface roughness of the compartments at both micron and submicron scales (as measured in the $5 \times 5 \mu \mathrm{m}$ and $1 \times 1 \mu \mathrm{m}$ areas, respectively (see Figure $5 \mathrm{~b}$ ). At the same time, the statistically significant difference in the stiffness of the compartment-specific ECMs was observed only via the measurements in the smaller regions of interest $(1 \times 1 \mu \mathrm{m}$ areas), while, it was not obvious at a larger scale (see Figure A d), which is due to the stronger influence of uneven surface topography in the areas $5 \times 5 \mu \mathrm{m}$ (see Figure A7 in Appendix A for the details). The detailed exploration of the surface roughness and stiffness distribution across different histoanatomical elements of both compartments is shown in Figure A8 in Appendix A.
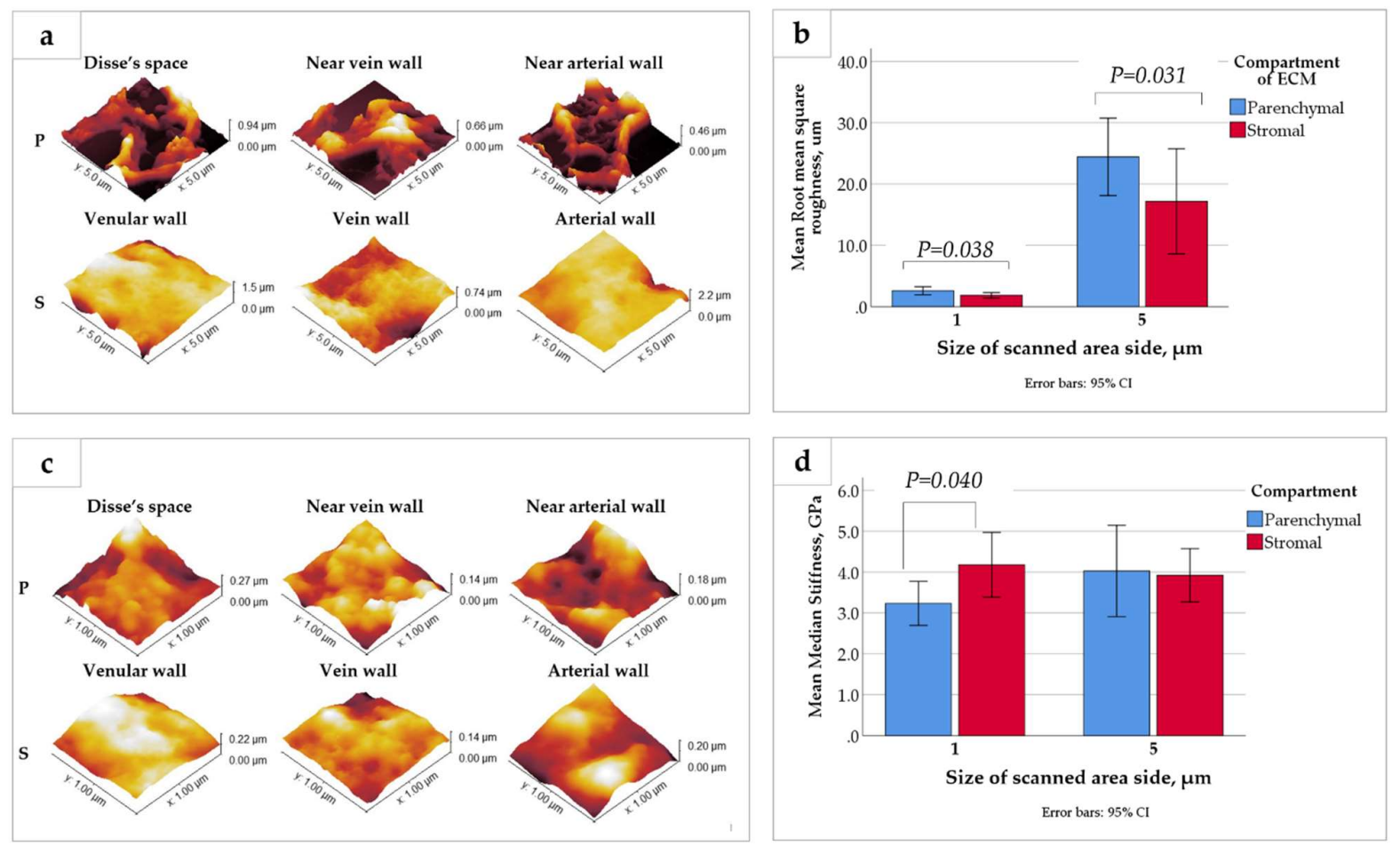

Figure 5. (a, c) Topography AFM images of the (a) micron scale, $5 \times 5 \mu \mathrm{m}$ and (c) submicron, $1 \times 1 \mu \mathrm{m}$ areas of the dehydrated CE liver ECM of the parenchymal (P) and stromal (S) origin. (b, d) Quantitative analysis of the surface roughness (b) and stiffness (d) of dehydrated LS-ECM compartments. Notably, the graph in (b) shows that the LS-ECM compartments differ by root square roughness at both micron and submicron scales (explored in $5 \times 5 \mu \mathrm{m}$ and $1 \times 1 \mu \mathrm{m}$ images, respectively) with a statistical significance. At the same time, as shown in (d), the LS-ECM compartments differ by median stiffness with a statistical significance only at submicron scale (as explored in $1 \times 1 \mu \mathrm{m}$ images).

While yet below the level of statistical significance, there were notable features of the ECM of vein walls and the parenchymal ECM near the vein walls. The vein walls' ECM showed the lowest roughness among all the studied histoanatomical structures at both micron and submicron levels, and it is the stiffest one at the micron scale. The ECM of parenchymal origin located near the vein walls has the highest stiffness and highest 
roughness in $5 \times 5 \mu \mathrm{m}$ samples (micron scale), while at the submicron scale it is the stiffest and the smoothest one among the ECM elements of parenchymal origin.

3.2.2. TNBC cells differentially colonize parenchymal and stromal LS-ECM, while ECM stimulates phenotypic plasticity of the cells

The histological study and digital image analysis of the liver-TNBC TECs over 4 weeks of in vitro culturing revealed distinctive patterns of initial cellular attachment and subsequent colonization in the parenchymal and stromal ECM compartments (Figure 6 a and b). In parallel, MDA-MB-231 cells contacting with different LS-ECM compartments exhibited increased phenotypic plasticity that was reflected in their morphology, specifically in the circularity. According to this, two subpopulations with different cell circularity were observed, as shown in Figure A9 in Appendix A. These subpopulations are further referred to as "epithelioid" (c 0.85) and "mesenchymal-like" (c 0.7) morphotypes.

The overall cellularity of the different compartments of the TNBC-liver TECs is shown in the Table A2 in Appendix A. The time course of the colonization of the liver AOSS by TNBC cell is described below.

During Week 1 of the in vitro TEC culture (see Figure 6 a, the top row), the number of cells attached to the ECM of the stromal compartment was 2.8 times higher than to the parenchymal ECM. Notably, at this time point, most cells in the in the stromal compartment formed single-row linings at the outer surfaces of the ECM, while the cells in the parenchymal compartment were mainly distanced from each other. In the parenchymal compartment a slight majority of cells had epithelioid morphology [59\% $( \pm 16 \%)]$, and the rest appeared mesenchymal-like. More than a half $[63 \%( \pm 10 \%)]$ of the cells observed in the stromal compartment had a mesenchymal-like elongated appearance.

At the end of Week 2 (see Figure $6 \mathrm{a}$, middle row and Figure $6 \mathrm{~b}$ ), in the parenchymal compartment, diffuse individual-cell colonization was predominantly $[75 \%( \pm 23 \%)]$ observed, while the remaining cells formed small multicellular clusters and single-cell surface linings over the AOSS surface. The individual cells mainly had the epithelioid morphology $[76 \%( \pm 7 \%)]$, and the rest were classified as mesenchymal-like. In the stromal compartment, cells mostly aggregated in multicellular clusters, both on the scaffold surfaces (as double- or triple-row linings or islands) and in the depth of the matrix. Only $17 \% \pm 21 \%$ of cells in the stromal compartment remained single. The invasion of the stromal ECM by the cell clusters occurred primarily along the voids and clefts in the scaffolds. These clusters invaded the stromal parts of AOSS to a depth (defined as a distance from the AOSS surface) of $70 \pm 24 \mu \mathrm{m}$, in contrast to the deeper $(134 \pm 54 \mu \mathrm{m})$ invasion by individual cancer cells observed in the parenchymal compartment. On the Weeks 2 to 3 , a tissue-like structure of the micrometastatic tumor was forming.

During Weeks 3 and 4 (see Figure 6 a, bottom row), a notable sponge-like remodelling of the ECM was observed in the stromal compartment, resulting in a blurred distinction between the ECM of the parenchymal and stromal origins. The invasion of cancer cells progressed over this period, reaching up to $800 \mu \mathrm{m}$ depth from the AOSS surface regardless of the matrix origin. By the end of Week 4, approximately $70 \% \pm 16 \%$ of single cells in all compartments retained their epithelioid morphology.

As mentioned above, starting from the first week of in vitro culture, MDA-MB-231 cells in 3D liver TECs formed two distinct subpopulations with epithelioid and mesenchymal-like shapes. Analysis of the distribution of the individual cells with epithelioid and mesenchymal-like morphotypes across parenchymal and stromal compartments during 4 weeks of in vitro culture is shown in Figure 6 c. According to the analysis of $95 \%$ confidence intervals for the proportions of the cell subtypes in the compartments, a statistically significant $(\mathrm{p}<0.05)$ dominance of the epithelioid cell shape (possibly, reflecting mesenchymal-to-epithelial transition, MET) was observed in the parenchymal compartment on the Weeks 2 and 4, and in the mixed compartment on Week 4. The statistically significant dominance of mesenchymal-like shaped cells (possibly, reflecting epithelialto-mesenchymal transition, EMT) was observed in the stromal compartment on Week 1. 


\subsubsection{Cell growth dynamics in $3 \mathrm{D}$ TECs is different vs. $2 \mathrm{D}$ cultures}

A comparative quantitative analysis of MDA-MB-231 cell growth in 2D monolayer cultures and 3D liver-TNBC TECs was carried out using the MTT assay (Figure $6 \mathrm{~d}$ ). The amount of viable cells that successfully attached to the surface of AOSSes on Day 1 was found to be $\sim 15-20 \%$ of the matching $2 \mathrm{D}$ culture seeded in plastic culture plates. This ratio corresponds to the ratio of the external surface area of the AOSSes $\left(\sim 0.4-0.8 \mathrm{~cm}^{2}\right)$ to the area of a well in a 24-well plate $\left(\sim 2 \mathrm{~cm}^{2}\right)$, indicating that the attachment efficiency in 3D TECs was approximately the same as on cell culture treated plastic and confirming high contact biocompatibility of the CE liver scaffolds.

The cells in 2D cultures and in TECs presented strikingly different growth behavior. As it is shown in Figure 6 d, cell density increased until Day 21 in both cultures, but with different rates. During Week 1, the growth observed in the 2D cultures was much faster than in the TEC counterparts. In the following 2 weeks, the weekly cell counts increased similarly in the 2D cultures and 3D TECs due to dramatic deceleration of growth rate in monolayers and slow but steady proliferation of cells in TECs. Importantly, the cell growth in TECs was stabilized between Weeks 2 and 3, indicating the most reliable and reproducible phase of the $3 \mathrm{D}$ culture model structural evolution combined with high viability/predictable numbers of the cells per construct which is needed for drug and nanoparticles testing and many other potential applications. As it discussed above, at this stage TECs were acquiring the tissue-like structure of the reconstructed micrometastasis.

In final Week 4, the growth rates in both types of cultures decreased in comparison to that of Week 3. This decrease was less pronounced in the TECs. Between Days 21 and 28 in vitro, the cell viability in 2D cultures decreased by approximately $19 \%( \pm 10 \%)$; and in the TECs - by $8 \%( \pm 36 \%)$. Differences in viability of cells between $2 \mathrm{D}$ and TEC cultures were statistically significant at each time point $(\mathrm{p}<0.001)$.

The observed cell population dynamics in the matching 2D and 3D in vitro cultures of MDA-MB-231 cells closely followed a general logistic growth model (see Equation 1 in Materials and Methods). The cell population growth rate (d) in 3D TECs was lower compared to the growth rate in the $2 \mathrm{D}$ cell culture $\left(d=0.28\right.$ days $^{-1}$ in $3 \mathrm{D}$ vs $d=0.63$ days $^{-1}$ in 2D). The results of the fitting and the estimated values of the model parameters are provided in Tables A3 and A4 (Appendix A), respectively.

Interestingly, while the overall cellular growth in the TECs (estimated using the biochemical MTT assay) followed the logistic model, the subpopulation of the cells localizing near the scaffolds surfaces (within the outer $300 \mu \mathrm{m}$ of the ECM), as measured via digital image analysis, behaved differently and grew linearly with the time (Figure A10). 

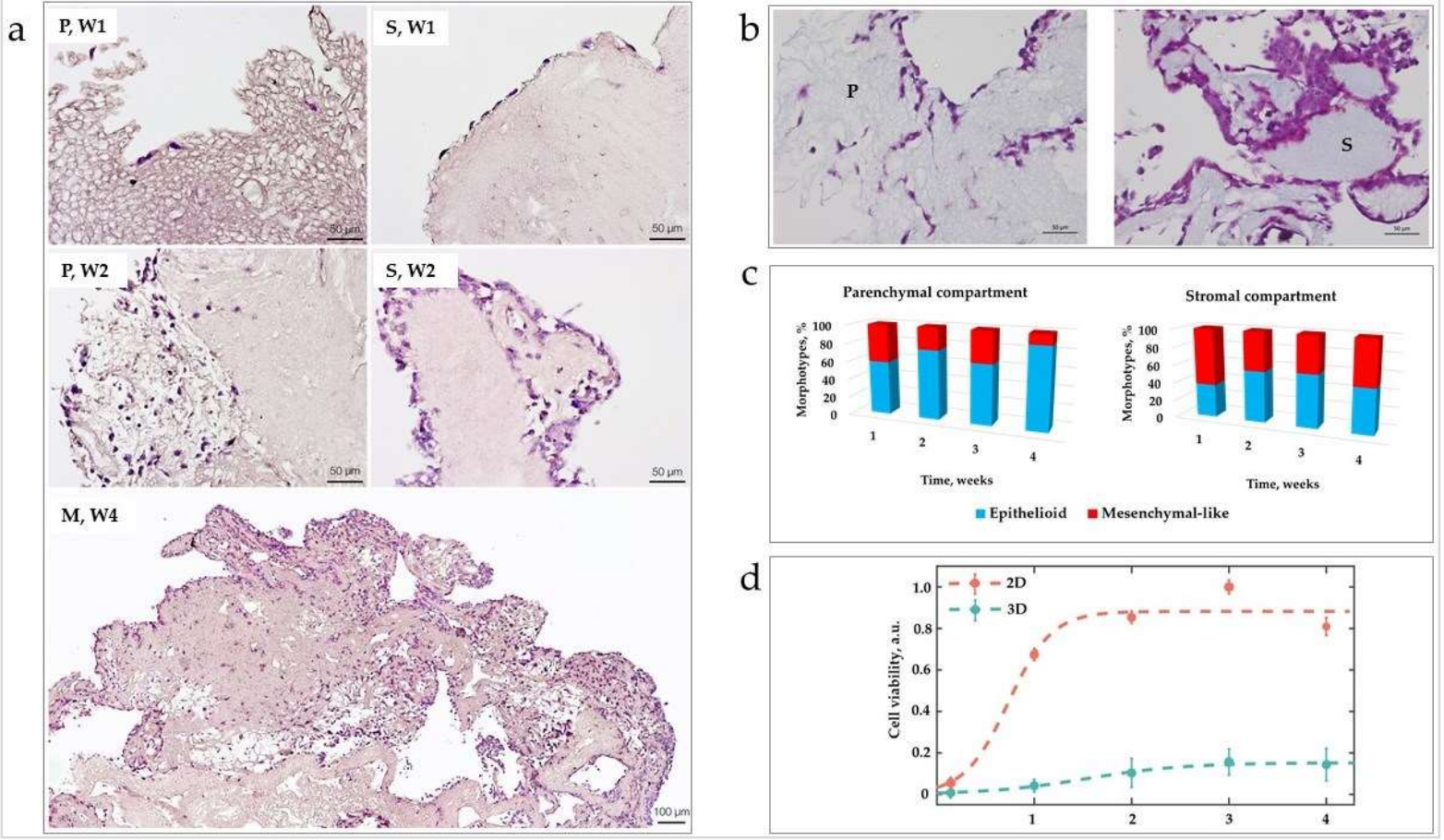

Figure 6.

(a) Representative histological images of cellular colonization of the liver AOSS during 4 weeks of in vitro culture (time points are labelled as W1, W2, and W4 for the first, second and fourth weeks, respectively. The images of parenchymal and stromal compartments are labelled as " $\mathrm{P}$ " and " $\mathrm{S}$ ", respectively. " $\mathrm{M}$ " labels the remodelled ECM with the mixed parenchymal and stromal features. Staining with H\&E, scale bars $50 \mu \mathrm{m}$ (top and middle rows) and $100 \mu \mathrm{m}$ (bottom row). Top row shows the colonization patterns on the $5^{\text {th }}$ day in vitro. Note sparse individual cells that are attached to the loose spongy-like parenchymal ECM on the outer border of the TEC (on the left). In contrast, formation of a discontinuous lining is visible on the dense ECM of the stromal compartment (on the right). Middle row,

Day 13 in vitro: note the colonization of the parenchymal compartment $(\mathrm{P}, \mathrm{W} 2)$ (the fragment with loose structure near the surface of AOSS) by individual cells and predominantly single-cell invasion to the stromal ECM. A few small multicellular clusters showing predominant single-cell invasion in the deeper parts of the scaffold are visible on the border with the area composed by the stromal ECM. In p0arallel, in the stromal ECM compartment (S, W2): the continuous multi-row cell lining on the stromal ECM with minimal invasion is observable. Bottom row (M, W4): remodeling of the ECM of stromal origin and massive diffuse colonization of the whole scaffold (Day 28). (b) Colonization of the surface parts of the parenchymal and stromal CE liver ECM by TNBC cells. (b) Parenchymal (P) and stromal (S) compartments of 3D TECs on the Week 2. Staining by Masson's trichrome method. Note similar tinctorial properties (light blue staining indicating comparable concentration of collagen) and different distribution of the cells (stained purple) over the compartments. There are lower cell numbers and deeper invasion of individual cells in the parenchymal sector and formation of dense cellular clusters near the surfaces (with shallow invasion depth of cell collectives) in the stromal compartment. Scale bars: $50 \mu \mathrm{m}$. (c) Effect of parenchymal and stromal LS-ECM on cell morphology. Distribution of the epithelioid and mesenchymal-like cellular morphotypes in LS-ECM compartments during 4 weeks of in vitro culture in TECs. (d) Cell growth dynamics of MDA-MB-231 cells in 2D and 3D (TEC) in vitro cultures. Dash curves represent the lines of best fit given by Equation 1.

\subsubsection{D TECs are angiogenic in vivo}

The angiogenic potential of the TNBC-liver TECs and CE liver AOSSes in vivo was examined using the chick embryo CAM assay as described in the Appendix A, Section A2.4 and shown in Figures A11-A14. We found that grafting of CAM with 3D TECs, liver AOSSes and suspensions of MDA-MB-231 cells induced different changes of natural angiogenesis in CAM occurring between ED8 and ED12 and resulting in specific architecture of blood vessel trees in the studied groups (Figure 7 and Figure A13). The convergence on 
the host blood vessels towards the graft was clearly visible in CAMs grafted with TECs, but it was less obvious in other groups (Figure A14).

The TECs and cell xenografts induced statistically significant increase of branch length density in CAM blood vessels, in comparison to the intact control, while grafting of the AOSSes did not result in this angiogenic switch (Figure 7 (a-f) and Figure A14). In contrast to other types of xenografts, TECs also induced intensive branching of blood vessels (Figure $8(\mathrm{~g})$ ), accompanied by the formation of long torturous segments between the branching points (Figure A13 (c)) and resulted in increased total blood vessel density (Figure A13 (a)). At the same time, the elongation of the branching blood vessels in CAMs grafted with TECs and scaffolds was rather inhibited as demonstrated in Figure 7 (c) and 7 (e). The combination of these changes resulted in formation of the areas of denser blood vessels networks between the proper branching points. Finally, as shown in Figure A13, in TECs-grafted CAMs, the total blood vessel density and segment length per area were increased in comparison to all other groups.

Figure 7 (f) shows the empirical cumulative distribution functions (ECDFs) visualizing the branch length density distributions in intact CAMs on ED8 and in each of 4 groups on ED12 which summarize the data presented in Figure 7 (a-e). These results show that compared with the measurements on ED8, the CAMs that developed naturally underwent detectable angiogenesis, while the CE liver AOSSes did not enhance this at the level of statistical significance. At the same time, the TECs supported the development of higher branch length densities in CAMs than that occurred in the naturally developing embryos and in CAMs grafted with the unseeded scaffolds (Figure $7(\mathrm{~g})$ ). The most pronounced increase in the branch length density was detected in the Cells group.
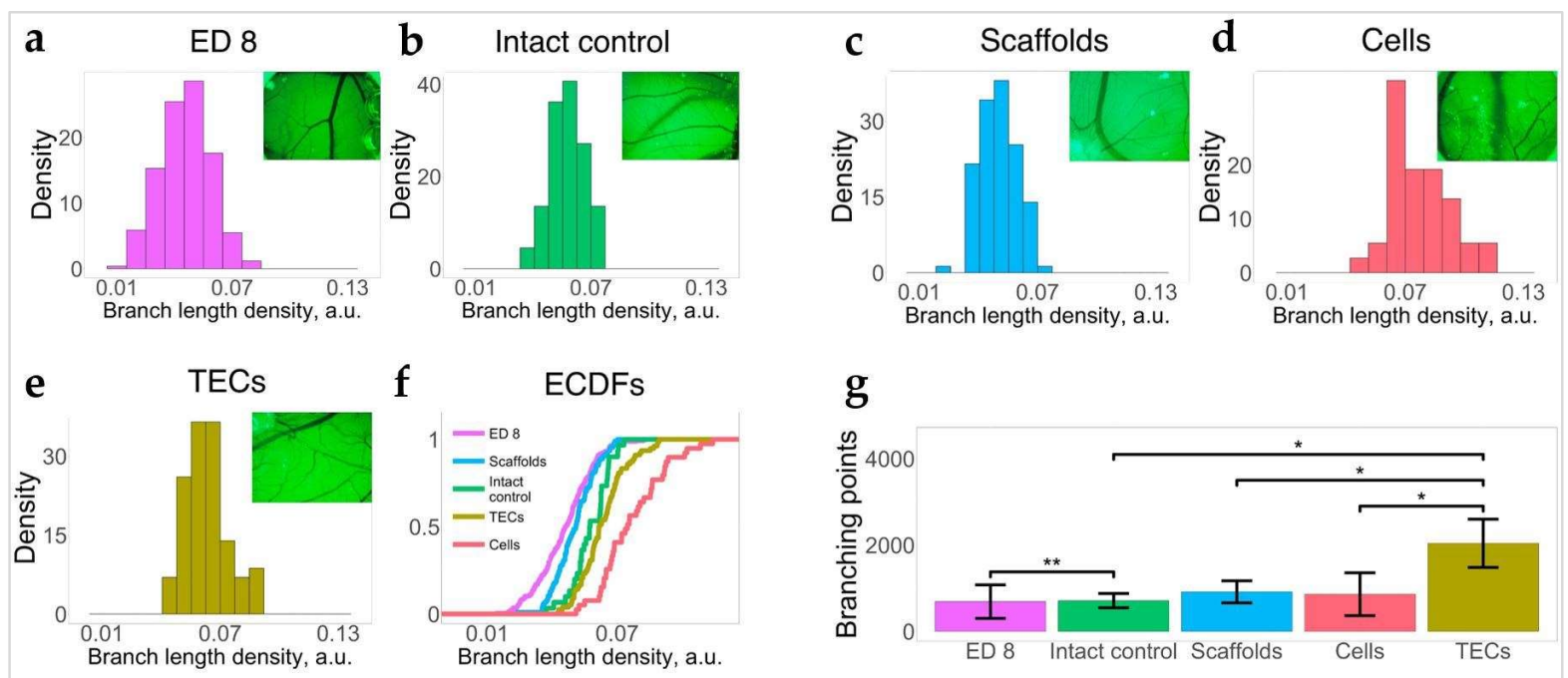

Figure 7. The angiogenic effects of 3D TECs, liver AOSSes (Scaffolds), and suspension of MDA-MB-231 cells (Cells), grafted on a chick embryo CAM compared with natural growth of CAM vasculature (Intact control) in the period between ED8 and ED12 of the embryonic development. Histograms of branch length density of (a) CAM vasculature on the day of grafting (ED8), and in (b) Intact control CAM (ED12), (c) Scaffolds, (d) Cells and (e) TECs. The inserts are images of blood vessels located in the vicinity of grafted materials. (f) ECDFs of branch length density in CAMs on ED8 and in each group on ED12. (g) Comparison of the number of branching points (per unit area) in CAM blood vessels in the studied groups. Error bars indicate standard deviation. Stars denote the level of statistical significance by Mann-Whitney test ${ }^{*} \mathrm{p}<0.05,{ }^{* *}$ $\mathrm{p}<0.01$.

\subsection{The 3D TNBC-liver TECs as testbeds for drug and nanoparticles testing}

3.3.1. TNBC cells in 3D TECs are less sensitive to the cytotoxic action of Dox and AMS-6Dox than in 2D cultures of MDA-MB-231 cells

The cytotoxic effect and cellular uptake of a chemotherapeutic drug Doxorubicin (Dox) was evaluated in parallel in 3D TECs and 2D monolayer cultures of MDA-MB-231 
cells. The Dox was applied to 3D and 2D models of TNBC in equal concentrations. It was delivered to the cells either in free (molecular) form or by anionic surfactant mesoporous silica nanoparticles loaded with Dox (AMS-6-Dox).

AMS-6-Dox were prepared and carefully characterized, as described in the Methods, and the results are shown in Figures A15-A20 and Table A5 in Appendix A. The results of MTT viability assays performed in 2D and 3D TEC cultures incubated for $36 \mathrm{~h}$ in the presence of molecular and nanoformulated Dox are shown in Figure 8 and in Figure A21 (Appendix A). In a control study, we found that pristine AMS-6 nanoparticles were not cytotoxic in both 3D TECs and 2D cultures at a concentration range $0-250 \mu \mathrm{g} / \mathrm{mL}$, which is consistent with our previous studies [58].

The cells in 3D TECs appeared much more resilient to both molecular and nanoformulated Dox in comparison with cells in 2D cultures (Figure 8 a, top graphs). The doseresponse relationship in each group was described, using a sigmoid function (see Methods for details, Equation 3). A half maximum effective concentration, EC50, in 3D TECs was found to be approximately 9 times higher than in 2D cultures for both formulations (see Tables A6 and A7 in Appendix A). Moreover, in 2D culture, the half maximum inhibitory concentration, IC50, was found to be $0.43 \mu \mathrm{g} / \mathrm{mL}$ and $0.46 \mu \mathrm{g} / \mathrm{mL}$ for free and nanoformulated Dox, respectively, while in 3D TECs, 50\% cell death threshold was not reached, indicating a markedly worse efficacy of both formulations in TECs compared to conventional 2D cultures (Figure 8 a, bottom graph).
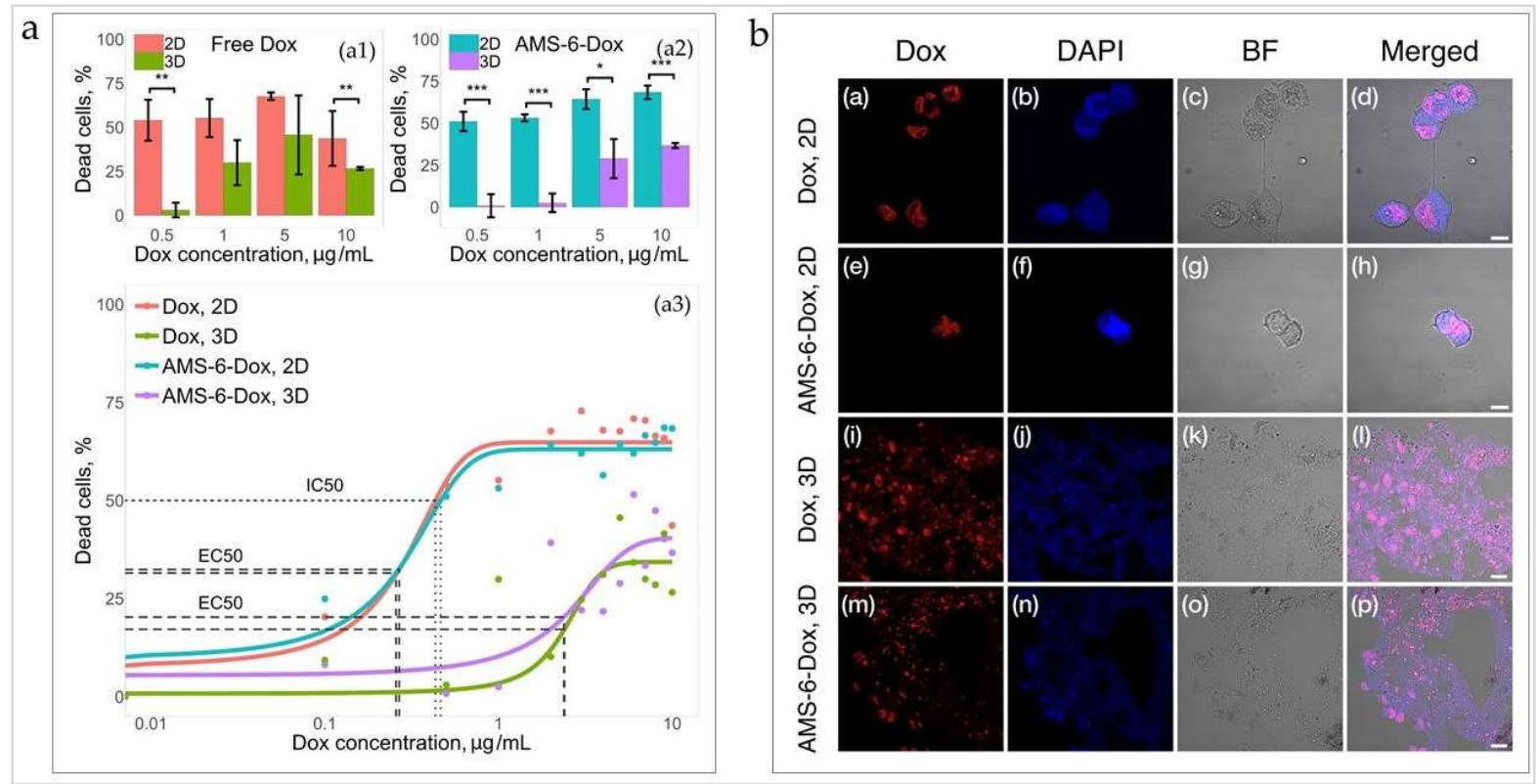

Figure 8. (a) Cytotoxicity of free and nanoformulated Dox in 2D cultures of MDA-MB-231 cells and 3D TECs. The top graphs: The effect of free Dox (on the left) and the effect of AMS-6-Dox (right graph). Error bars indicate 95\% CIs; statistically significant difference at ${ }^{*} \mathrm{p}<0.05,{ }^{* *} \mathrm{p}<0.01,{ }^{* * *} \mathrm{p}<0.001$ by Mann-Whitney U test. Bottom graph: Dose-response curves of free Dox and AMS-6-Dox in 2D and 3D in vitro cultures. Solid lines represent the lines of best fit given by Equation 3. (See Tables A6 and A7 for fit parameters). Note that in the 3D culture, the IC50 could not be calculated within the studied concentration range (up to $10 \mu \mathrm{g} / \mathrm{mL}$ ). (b) Confocal microscopy images of MDA-MB-231 cells incubated for $24 \mathrm{~h}$ with $(\mathrm{a}-\mathrm{d})$ Dox in 2D; $(\mathrm{e}-\mathrm{h})$ AMS-6-Dox in 2D; $(\mathrm{i}-\mathrm{l})$ Dox in 3D; and $(\mathrm{m}-\mathrm{p})$ AMS-6-Dox in 3D TECs. Intrinsic Dox fluorescence was detected in the red channel (Dox), while DAPI fluorescence (blue channel, DAPI) was used for contrasting of cell nuclei. Control bright field (BF) images were acquired to visualize the tissue structures, and merged images highlight the colocalization of Dox and DAPI signals. Note the absence of preserved cell nuclei in the depth of TEC treated with AMS-6-Dox nanoparticles (n), in comparison with TECs treated with free Dox (j). Dox concentration, $10 \mu \mathrm{g} / \mathrm{mL}$. Scale bars, $10 \mu \mathrm{m}$ (Dox, 2D; AMS-6-Dox, 2D) and $20 \mu \mathrm{m}$ (Dox, 3D; AMS-6-Dox, 3D). 
The data also shows the effect of Dox delivery formulation (free or nanoformulated) on the cellular response in both 2D and TEC cultures (Figure 8 a), and Figure A21 in Appendix A). Nanoformulated Dox was generally more cytotoxic at higher Dox concentrations (>5 $\mathrm{gg} / \mathrm{mL}$ ), while the efficacy of free Dox was reduced at the higher Dox concentrations in both 2D and 3D cultures. At a maximum studied Dox concentration of $10 \mu \mathrm{g} / \mathrm{mL}$ the difference in mean viability between $3 \mathrm{D}$ and $2 \mathrm{D}$ cultures was $17.1 \%$ and $31.7 \%$ for Dox and AMS-6-Dox, respectively (see Figure 8 a).

\subsubsection{Uptake of Dox and AMS-6-Dox in 3D TECs and 2D cultures of MDA-MB-231 cells}

The results of evaluation of cell and tissue uptake of Dox and AMS-6-Dox in 2D and 3D TEC cultures are shown in Figure $8 \mathrm{~b}$ and Figure A22. In 2D cultures, the fluorescent signals of free Dox and AMD-6-Dox nanoparticles were visible in the cellular nuclei (Figure $8 \mathrm{~b}(\mathrm{a}-\mathrm{h})$ ). In contrast, in 3D TECs, fluorescence signals of free and nanoformulated Dox were distributed between the cell nuclei, cell cytoplasm and ECM of TECs. Free Dox was noticeably accumulated not only in the cell nuclei, but also throughout the TECs' ECM, while fluorescence from AMS-6-Dox in 3D TECs was mainly detected in the nuclei of cells located at the TEC surface and only diffuse DAPI staining and Dox-positive cell debris was observed at a depth of $>50 \mu \mathrm{m}$ in TEC, indicating destroyed nuclei and destroyed tumor cells, respectively (Figure $8 \mathrm{~b}(\mathrm{i}-\mathrm{p})$ ).

To sum up, the MTT test and imaging results show that the distribution and cytotoxic effects of free and nanoformulated Dox were influenced by the liver-specific ECM microenvironment, and the method of Dox delivery to 3D TECs modulated its local therapeutic activity.

\section{Discussion}

In this study, we developed and tested a new approach for the creation of organspecific tissue engineered $3 \mathrm{D}$ in vitro models of cancer micrometastases using the CE experimental platform. First, we demonstrated successful preparation of the panel of AOSS obtained by iWO-DCL protocol and their recellularization with human TNBC cells of MDA-MB-231 line. Then, by the comparative analysis of colonization patterns of the multiple organ AOSS we examined the role of the organ-specific ECM in the modulation of the risk of TNBC metastatic colonization of the secondary organs. Next, we established a new model of early metastasis of TNBC to the liver using our proposed tissue engineering approach, and then we exemplified the applications of this model for basic cancer biology research and for drug and nanomedicines testing.

The CE tissue engineering platform for disease modelling is inexpensive and highly reproducible as the CEs as tissue/organ sources can be obtained from industry poultry suppliers, implying low cost and an exceptionally high level of control/infection-free production [59]. In terms of the economical effectiveness, our estimations show that, for example, 100-150 scaffolds can be prepared from a single liver of an ED18 broiler CE, resulting in the cost price per experimental object of about $0.01-0.02$ USD. The price cost of the CE AOSS derived from the other organs may be higher due to the lower volume of the organs or/and the ECM content. However, it may be an additional (besides the obvious ethical considerations) motivation for the replacement of the experiments on mice and rats (estimated cost per animal is about $\$ 7$ or $\$ 20$, respectively) with CE tissue engineering products in certain areas of research and development. The proposed iWO-DCL procedure avoids the complexities of the perfusion-based WO-DCL, while allowing decent quality whole-organ scaffolds preparation by a simplified immersion-agitation procedure. We demonstrated that iWO-DCL is efficient for the preparation of a variety of highly biocompatible AOSS with long shelf life. The presented model allows maintaining the engineered 3D tumors in vitro for long periods (at least 1 month, as demonstrated in this work), which is important for metastatic cancer research. We demonstrated the applicability of the similar to iWO-DCL protocol for the creation of AOSS and TEC based on the 
food-grade liver of mature chickens [60]. However, the last approach does not allow to prepared the multiorgan AOSS panels and control the source of the tissues.

The TECs may also be transferred to in vivo settings as in vitro pre-cultured controllable tumor xenografts, offering a unique possibility to observe intrinsic mechanisms behind metastatic cancer outside and inside the body. Notably, the panels of AOSS can be prepared from the flocks of animals with high genetic similarity without using the specialized vivarium setups and procedures. Moreover, the immaturity of the immune system and pain sensitivity of the $\mathrm{CE}$, as well as the easiness of manipulations on the extraembryonic membranes [61,62], makes $\mathrm{CE}$ a promising experimental system for grafting of the tumors reconstructed by tissue engineering (3D TECs) on CAM, as it has been demonstrated in our study. Taken together, these arguments indicate that the proposed CE platform for the 3D tissue engineering modelling of tumors is an attractive option for high-throughput research and pharmaceutical applications.

Beyond these methodological insights, our study has revealed a series of interesting findings in cancer biology.

First, using the multiorgan panel of CE AOSS, we explored the role of organ-specific ECMs in metastatic organotropism. Our tumor of interest was TNBC. TNBC is an aggressive HER2-, estrogen and progesterone receptor-negative mammary carcinoma [63-65]. Lack of targeted therapy and limited efficiency of conventional treatment (performed mainly by chemotherapy with cytostatic drugs) contribute to the low survival of the patients with metastatic TNBC [66].

It is known that by autopsy data, breast cancer most frequently metastasizes to the following organs listed in descending order: lymph nodes, lungs, liver and bones [67]. TNBC has one of the highest propensity to distant metastases [68] [69]. The most common places of the distant metastases of TNBC are lungs (31\%), bone (27\%), liver (15\%), central nervous system (15\%) and pleura (12\%) [70]. It is also reported that TNBC more frequently than many other molecular subtypes of breast cancer metastasizes to the visceral organs [13] / abdominal cavity [68]. We used a line of highly invasive TNBC cells (MDA-MB-231), which is considered to possess EMT-associated mesenchymal-like phenotype in vitro [71]. Our results quantitatively demonstrated a similar pattern of the "preference" of the organspecific ECM by these cells in terms of attachment and colonization efficiency. The lungs ECM emerged as the statistically significantly advantageous substrate for the TNBC cells growth, in comparison with the brain. The ECM of the heart was the least "preferred" one. The efficiency of the colonization of the LS-ECM was slightly less than in the lungs scaffolds and slightly higher than in the brain AOSS (the difference did not reach the level of statistical significance). Then, our observations show that the organ-specific ECM alone can be the guiding factor for the preferred TNBC metastatic homing or colonization - considering the pair of the lungs and the brain. LS-ECM takes an intermediate position in terms of the efficiency of colonization, between the lungs and brain. This finding is coherent with the reported clinical and autopsy data on the TNBC organotropism $[67,68,70]$. We also confirm that brain ECM as well as the ECM of other CE organs can be used as AOSS for 3D culture of MDA-MB-231 cells. In a pilot study with adding a decalcination step to the iWO-DCL we also obtained CE bone AOSS and successfully recellularized it with the same cells (Figure A23, Appendix A). As the interactions between lung-specific ECMs obtained by DCL with breast cancer cells were analysed in recent publications $[28,72]$, in our study, we chose to focus on the detailed analysis of the hepatic micrometastases of TNBC.

Liver is a common place of the metastatic spread for many types of cancer, including breast cancer [11]. In particular, breast carcinomas are the most frequent primary cancer site in young women (20-50 years of age) with liver metastases, and this location indicates a pure prognosis [73]. The causes of such liver "preference" of the TNBC metastases are virtually unknown. As the role of blood inflow is rather comparable to other carcinomas not associated with the digestive tract, the local tissue-specific mechanisms are hypothesized to be a key factor $[8,74]$. For instance, special organization of hepatic vasculature, 
such as discontinuous basement membranes in sinusoids, allows extravasation of metastatic cancer cells to occur first in the narrow $(\sim 1-2 \mu \mathrm{m})$ gap between endothelial cells and hepatocytes, known as Disse's space [75-78]. It forms the major part of ECM framework of hepatic parenchyma and contains blood plasma, collagens types I, III, V, VI and VII, as well as fibronectin and tenascin [79]. Therefore, hepatic ECM (see Figures A4 and A5 in Appendix A for illustration) makes the first homing space for the incoming cancer cells $[75,80,81]$ and becomes the potential starting point, the operational environment and the regulator of metastatic colonization of the organ $[78,82,83]$.

The iWO-DCL methodology introduced here maintained the liver-specific ECM histoanatomy with identifiable parenchymal and stromal compartments. It also preserved major molecular components of the ECM such as fibrillar collagen as indicated by VanGieson's and Masson's trichrome staining (see Figures $3 \mathrm{~b}$ and $4 \mathrm{~b}$ ) in the same way as it has been previously reported for perfusion whole-organ DCL of the liver $[24,55-57,84]$. Therefore, iWO-DCL liver AOSSes form biologically accurate scaffolds reproducing the organ-specific (in particular, the hepatic) microenvironment more closely in comparison with synthetic scaffolds and culture plastic, both lacking natural signalling moieties and tissue architecture.

One of the key observations of the current study is the existence of two compartments with different properties in the CE liver ECM as revealed by iWO-DCL. We performed a pilot study of these properties and found that the stromal compartment contains more fibrous collagen than the parenchymal one (see Figure $3 \mathrm{~b}$ ), and the architecture of the two compartments are essentially different (see Figure 3 a). Considering the ubiquitous presence of collagen in the liver AOSS and the confirmed preferential attachment of the MDAMB-231 cells to collagen type I and fibronectin demonstrated by us recently [85], we further concentrated on the analysis of the physical features of the parenchymal and stromal ECM of the liver. Using AFM, we show that Young's modulus of the CE liver scaffolds stored in PBS was about $0.2 \mathrm{kPa}$ which is close to the previously reported values for the abdominal organs, including the liver [86]. However, we could not discern the specific mechanical characteristics of the parenchymal and stromal ECM in hydrated AOSS due to the extensive surface topography. Therefore, we applied our original methodology [47] and examined the dehydrated and deparaffinated unstained histological sections of the CE liver AOSS by AFM. This allowed to define that the two liver ECM compartments differ at both micron and submicron scales in their roughness (the parenchymal ECM is rougher) and at the submicron scale in their stiffness (the stromal ECM is stiffer). The obtained stiffness values (in the GPa range) are considered to be characteristic to collagen fibrils [86].

In the current study, we found that upon the first contact with the liver ECM, TNBC cells preferentially attached to the matrix of the stromal compartment, in comparison to the almost three times less frequent binding to the parenchymal ECM. We think that this indicates that the perivascular ECM forms the most attractive niche for TNBC cells, while the initial entrance of the disseminating cancer cells happens via the Disse's space ECM (parenchymal compartment of LS-ECM). The concentration of collagen as well as the relatively larger available space for attachment (extended smoother surfaces) of the stromal ECM of the liver, may be probable drivers of the selective higher seeding of TNBC cells in the stromal compartment.

Next, during the first two weeks of culturing, the cells in the parenchymal compartments mainly remained individual and dispersed in the spongy-like ECM of the former parenchyma. At the same time, the stromal compartments were populated mostly by multicellular clusters which lined along denser connective tissue elements. The observed cellular distribution is consistent with the reported finding that the earliest detection of nonvascularized hepatic metastases, which presented as cellular clumps in Doppler sonography, was possible near the portal triads (i.e., in the stromal compartment) [87]. The observed pattern of TNBC cells dissemination across LS-ECM compartments also explains the negligible detectability [7,88-90] of single-cell occult metastases in the liver parenchyma vs the cell clusters near blood vessels and other stromal components. 
During the first two weeks of observations, the initial individual vs clustered cell adhesion in the parenchymal and stromal ECM compartments was further reflected in their subsequent differential invasion patterns. The features of TNBC cell invasion in the parenchymal compartments can be attributed to amoeboid migration [91] as the cells mainly preserved their round epithelial morphology and appeared to be separate from each other, permeating through small ECM voids without histopathologically visible signs of matrix degradation. The stromal compartments were initially invaded by clusters of cancer cells, therefore indicating that collective cell migration [91] was the early mechanism of the colonization in the stromal ECM. These alternative colonization strategies resulted in different depths of the invasion fronts in the parenchymal and stromal compartments, found to be $134 \mu \mathrm{m}$ and $70 \mu \mathrm{m}$, respectively, implying that individual cell migration in the former hepatic parenchyma resulted in a faster speed of the invasion front ( $\sim 10 \mu \mathrm{m} /$ day in the current experimental conditions), while the stromal parts of the liver matrix were invaded more slowly ( $\sim 5 \mu \mathrm{m} /$ day) by TNBC cellular cohorts. This is consistent with the in vitro data indicating that individual cancer cells migration is faster than the collective mode due to different adhesion mechanisms and reactions to spatial confinement [92]. The cellular density in the parenchymal compartment by the end of the second week after seeding remained 2.4 times lower than in the stromal zones, indicating, probably, that proliferation and migration states of the cells were, to a certain extent, were mutually exclusive and/or spatially separated. Interestingly, a similar phenomenon was observed in malignant gliomas [93]. We conclude that early TNBC colonization of the parenchymal and stromal compartments of the liver ECM occurs via different mechanisms and may require different diagnostic and therapeutic approaches. In particular, it the increased detection sensitivity and depth is needed to reveal the micrometastases hidden in the liver parenchyma in combination with using of the cellular immobilization, rather than suppression of the proliferation. The cytostatic treatment needs to be used first of all in the stromal part. Therefore, the development of compartment-specific delivery agents, for example, targeted nanomedicines, for the selected drugs with differentiated mechanisms of action may be an efficient way to control liver metastases of TNBC.

From Week 3 onwards, after the significant remodelling of the stromal ECM causing its loosening and gradual blurring of a structural difference between the liver ECM compartments, the scaffolds were invaded to the depth of $800-1,500 \mu \mathrm{m}$ from the surface, while the cellular population comprised a mix of cells with epithelioid and mesenchymallike morphologies. At this stage, a significant increase of the invasion front speed was observed (to $\sim 26-50 \mu \mathrm{m} /$ day) and associated with reversible phenotype transitions (from epithelial- to mesenchymal-like and vice versa) and the matrix remodelling employed by TNBC cells as colonization strategies. This is in agreement with the reported findings of the interdependence of the migration mode and morphology of the cells, and transition between the mesenchymal and epithelial phenotypes in the migrating cellular clusters resulting in individual dissemination of epithelial-like cells [94].

The mechanisms underlying the observed differential patterns of adhesion and invasion of TNBC cells in the parenchymal and stromal compartments of the liver ECM are yet to be explored. The role of a number of factors is to be addressed in future studies, and the first thinkable two candidates, to our vision, are the spatial gradients $[95,96]$ of ECM components between the triads, central veins and Disse's space (as it is illustrated and commented in Figures A4 and A5); and the density, stiffness [97-99] and roughness (as observed in the current study as well), the porosity or confinement $[100,101]$ of the matrix space of hepatic parenchyma and stroma. These properties of ECM are non-decouplable in the current model system [102] and require intentional reduction of the complexity of the operational environment of the cells. As an intentional limitation of the current study, we have not addressed the precise spatial distribution of the ECM proteins in the scaffolds due to the limited availability of reliable immunohistochemical (IHC) tools that reproducibly work in avian tissues and the intrinsically non-quantitative nature of IHC studies. However, we confirmed the significance of the ECM protein composition for the MDAMB-231 cells attachment in an experiment on the artificial controlled ECM microarrays 
and revealed preferential adhesion to collagen type I and fibronectin-containing substrates and moderate to low cells' attachment to the components of basement membranes in particular [85]. Moreover, in the current research, we observed the changes of the cell shapes depending on the contact with the parenchymal and stromal ECMs. The epithelioid morphotype dominated in the parenchymal compartment, while the mesenchymallike shapes transitionally emerged mostly in the stromal compartment. Importantly, it was notable that the studied TNBC cells demonstrate very high phenotypic plasticity and rather swinging than switching between epithelial and mesenchymal-like morphology, possibly reflecting the fluctuations between the epithelial-to-mesenchymal transition EMT) and mesenchymal-to-epithelial transition (MET), when they were colonizing different compartments of the LS-ECM. This corresponds well with the concept of partial EMT as a mechanism of cancer cells adaptation to the specific niches [103] and the very important mechanism of the metastatic organotropism [12]. The current observations are also in compliance with our findings on the effect of the collagenous substrates of different stiffness on the morphotypes and EMT/MET transitions in MDA-MB-231 cells [85].

Our results show essentially different cell population dynamics in the 2D culture of MDA-MB-231 cells and in 3D TECs. While the initial amount of attached cells per area was similar between the 2D plastic substrates and liver AOSSes, the plastic-cultured cells grew much faster during Week 1 than cells in the 3D TEC. Later, in 2D culture, the growth rates stabilized and even decreased in Week 4, while in 3D TECs the cell viability was steadily increasing during the first 3 weeks. We found that a logistic growth model was applicable to both cultures. Interestingly, such model accurately captured the growth of breast carcinoma cells in large clinical datasets [104]. By its nature, the logistic growth mode indicates the presence of resource limitations [105]. We think, in 2D cultures, this was likely to be due to the saturation of the cell attachment capacity of the wells which has been reached on the first week (Figure $4 \mathrm{~d}$ ). This was not the case for the TECs because of the initially smaller numbers of attached cells, and larger total surface area of the porous ECM scaffolds. We attribute the decrease of cell growth rate in TECs after Week 3 to metabolic limitations of 3D cell culture, such as relative hypoxia at the depth of the constructs [106]. This agrees with our histological observations of the secondary necrotic areas forming at the late stages of TECs development. This is additionally supported by the observation of the linear cell growth near the surface (the part with better oxygenation and nutrition) in 3D TECs (see Figure A10 f in Appendix A).

We also validated our tissue engineering model of TNBC micrometastases to the liver in vivo by an angiogenic assay on chick embryo CAM. The angiogenic switch is the key limiting process for a transition between micro- and macrometastatic states [6]. We found that grafted TECs induced an enhanced angiogenic response in comparison with natural embryonic CAM vascularization. In contrast to natural embryonic angiogenesis, which occurs within the studied incubation age mainly by intussusceptive microvascular growth (i.e. splitting of the existing capillaries along the blood vessel axis) [107], the grafted tumors stimulated branching of the host blood vessels, indicating that sprouting angiogenesis was taking place. Interestingly, the increase of branching, in contrast to the increase of blood vessel length density, in comparison to controls, was not induced by grafted cell suspensions, indicating the contribution of the ECM-related factors to the tumor-specific angiogenic potential of the TECs. As the sprouting angiogenesis is the key part of the angiogenic switch in the metastatic progression[6,108], this result indicates that the presented model of early TNBC liver metastases behaves similarly to early metastasis.

Finally, we examined the feasibility of the 3D tissue engineering TNBC-liver AOSS model for drug and nanomedicine research. As shown recently, the use of nanoscale diagnostic and therapeutic agents is a promising approach to address the challenges of the detection and treatment of micrometastases [90]. We found that the sensitivity of TNBC cells to the chemotherapeutic drug Dox in free and nanoformulated forms was significantly reduced in 3D TECs, in comparison to 2D cultures, with 9-times increase of EC50 values and the value of over $10 \mu \mathrm{g} / \mathrm{mL}$ for IC50. This is in agreement with earlier reports that additional signalling and diffusion inputs from the 3D microenvironment and ECM 
lead to decreased responses to chemotherapeutics [109]. Another factor contributing to the reduced response on the cytostatic treatment is, probably, the difference in the growth rates between 2D and 3D model systems, linking the stronger cytostatic effect of Dox in the faster proliferating cells in 2D. We also observed a trend for a higher therapeutic effectiveness of the nanoformulated Dox in 3D TECs in comparison with free Dox, notable at high doses of doxorubicin (Figure A21). This result was corroborated by fluorescence imaging, where an uptake and intracellular distribution of free and nanoformulated Dox were found to be comparable in 2D TNBC cultures, while in 3D TECs the Dox released from AMS-6-Dox nanoparticles killed deeply located cells almost completely. In contrast, free Dox accumulated not only in the cell nuclei but also notably accumulated in the ECM (Figure $6 \mathrm{~b}$ ). We tentatively attribute this difference to the faster drug release from AMS6-Dox (see Figure A15 c in Appendix A) and electrochemically affected transport of the negatively charged AMS-6-Dox (see Table A5 in Appendix A) across the net-negatively charged LS-ECM. This observation additionally points on the potential reduction of the cytotoxic side effects of free Dox by using of the appropriate nanoscale delivery agents to prevent accumulation of this drug in the liver ECM. However, the detailed mechanisms of the observed phenomena were not the specific aim of the current work and require further studies.

\section{Conclusions}

In conclusion, the new engineered 3D micrometastatic cancer model investigated here fills the gap between conventional cell cultures and in vivo testing in animals, and it provides an affordable and facile platform for drug and nanomedicine development and cancer research. This model allowed us to reveal distinct, stage-specific and previously unknown ways of metastatic colonization of the liver by TNBC cells and contribute to the understanding of the mechanisms of metastatic organotropism. The compartmentalization of the liver ECM into dual parenchymal vs. stromal niches was found to be the key determinant of the colonization pattern used by TNBC cells. Cells cultured in 3D liverderived matrix demonstrated increased resilience to free and nanoformulated doxorubicin. The biological relevance of the model was confirmed in vivo by induction of angiogenic switch in the host tissue by the grafted TECs. As decellularized tissues have negligible cross-species differences and can be used as xenotransplants, this methodology is highly universal and may be applied to other types of chick embryo organs and other cell lines and tumor types.

\section{Patents}

The iWO-DCL procedure has been temporary protected by an Australian provisional patent No. 2018903766 by A. Guller, A. Nadort and E. Goldys (lapsed).

Author Contributions: Conceptualization, A.G. and A.Z.; methodology, A.G., I.K., V.R., A.N., Z.K. A.G-B., E.G., Y.Q., and A.Z.; software, I.K. and V.R.; validation, A.G., V.R., A.N., and A.P.; formal analysis, A.G., V.R., I.K., A.N. and A.Z.; investigation, A.G., I.K., A.N., Z.K., A.P. and Y.Y.; resources, Y.Q., E.G. and A.Z.; data curation, V.R. and A.N.; writing-original draft preparation, A.G., I.K., V.R. and A.N.; writing - review and editing, A.G., V.R., A.N., A.G-B., L.L., Y.Y., Y.Q., E.G. and A.Z.; visualization, A.G., V.R., I.K., A.N., L.L.; supervision, Y.Q. E.G. and A.Z.; project administration, A.G., Y.Q., E.G. and A.Z.; funding acquisition, Y.Q., E.G. and A.Z. All authors have read and agreed to the published version of the manuscript.

Funding: This research was partially funded by the ARC CENTRE FOR NANOSCALE BIOPHOTONICS CE14010003 and by the MINISTRY OF EDUCATION AND SCIENCE OF THE RUSSIAN FEDERATION (075-15-2019-1927) in part of the cell morphological analysis, and RUSSIAN SCIENCE FOUNDATION GRANT (No. 21-74-30016) in part of tissue matrices development. A part of the study was supported by IMQRES scholarship research funds by MACQUARIE UNIVERSITY (A.G.). A part of the study was also supported by Seed Grant awarded to A.G. by SYDNEY VITAL TRANSLATIONAL CANCER CENTRE and Pioneer research grant by TOUR DE CURE awarded to A.N., A.G., Y.Q., E.G. and A.Z. for the development of 3D tissue engineering cancer models. 
Institutional Review Board Statement: The study was conducted according to the guidelines of the Declaration of Helsinki, and approved by the Animal Ethics Committee of MACQUARIE UNIVERSITY (AEC Reference No.: 2015/006 approved on 16 $6^{\text {th }}$ of May 2015 with extensions for the next years of work) and by the Animal Care and Ethics Committee of the UNIVERSITY OF NEW SOUTH WALES (ACEC Reference No.: 19/103B approved on 22 $2^{\text {nd }}$ of August 2019 until 31 st of July 2022).

Informed Consent Statement: Not applicable.

Data Availability Statement: The relevant data generated and (or) analyzed in the current study is available from the corresponding author upon reasonable request.

Acknowledgments: A gift of fertilized chicken eggs from Baiada Poultry PTY Ltd is thankfully appreciated. We gratefully acknowledge discussions and critical review of histological results by Professor Anatoly Shekhter (Sechenov University). We are thankful to Ms. Larisa Grivans for her assistance with scientific illustrations. The authors acknowledge the facilities and the scientific and technical assistance of Microscopy Australia at the Electron Microscope Unit (EMU) within the Mark Wainwright Analytical Centre (MWAC) at UNSW Sydney. We thank Dr Celine Heu (UNSW) for her assistance in AFM measurements in wet samples. A.G. thanks to Macquarie University for providing an IMQRes scholarship and MQRF fellowship. Authors acknowledge the support by the Macquarie University Microscopy Unit and the everyday help of the Laboratory Operations Team of the Faculty of Medicine and Health Sciences Research Laboratory (Macquarie University).

Conflicts of Interest: The authors declare no conflict of interest.

\section{Appendix A}

A1. Design of the study on the feasibility of the 3D tissue engineered model of TNBC micometastses to the liver

The model design and applications are schematically illustrated in Figure 1. As a source of donor LS-ECM, we used chick embryos incubated until the embryonic day 18 (ED18) when all major organs have formed, while pain sensitivity is not yet fully developed [110]. After the extraction of the chick embryo livers, the organs underwent the iWODCL and then were cut by 4-mm biopsy punch to obtain acellular organ-specific scaffolds (AOSS). Each individual AOSS presented an 8-10 $\mathrm{mm}^{3} / 2-3 \mathrm{~mm}$ side size fragment of decellularized chick embryo liver allowing to preserve histoarchitectural features of the LSECM. Next, 3D TECs were created by seeding of MDA-MB-231 cells on the top of AOSSes placed in the individual wells of the culture plate. The cells grew on and within the scaffolds in a static in vitro culture for up to 4 weeks allowing us to characterize the cell invasion patterns, examine drug response and validate in vivo the cancer-specific behavior after implantation in chick embryos. The TECs were sampled for histological and other analyses at various time points during the in vitro culture (up to 4 weeks since cells seeding). Matching 2D in vitro cultures of the same cells were studied in parallel with the TECs. The TECs with well-developed cell colonies (after 3 weeks of in vitro culturing) were used for evaluation of cytotoxicity and cellular uptake of doxorubicin (Dox) as well as Dox-loaded mesoporous silica nanoparticles (Anionic Mesoporous Silica-6, AMS-6Dox) [111]. The ability of the engineered tumors to induce the "angiogenic switch", or growth of blood vessels in the host tissue, was examined by chick embryo chorioallantoic membrane (CAM) assay via grafting of the TECs [112]. This effect is known to be a critical cancer hallmark [113] especially significant for metastatic progression [4-6]. 


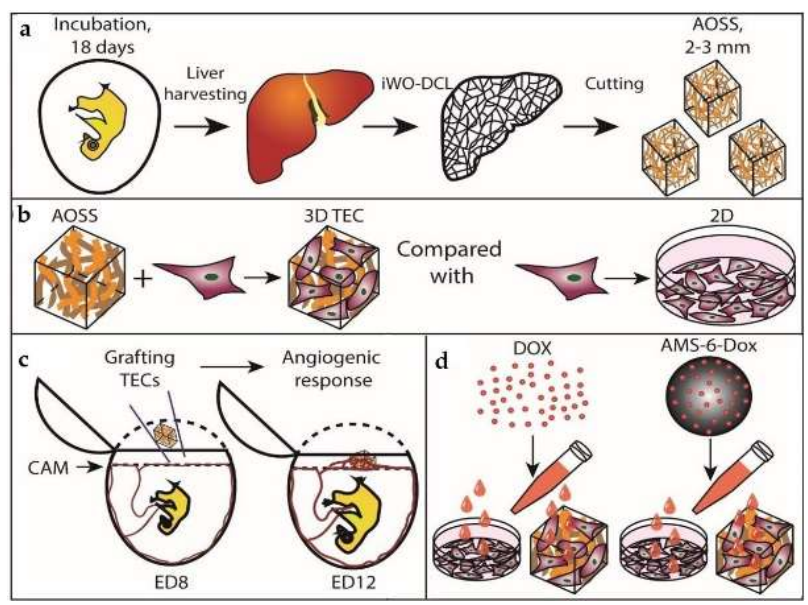

Figure 1. The scheme of study design. (a) Obtaining of the liver AOSS: incubation of CE and harvesting livers from ED18 CEs, followed by the iWO-DCL of the livers, and preparation of the AOSS by cutting decellularized livers into small fragments; (b) Preparation of 3D TECs by seeding of the liver AOSS with MDA-MB-231 cells; analysis of the colonization patterns and cellular geometries in TECs and comparison of cell population dynamics in TECs with conventional 2D cell cultures by MTT assay and histopathological examination; (c) Evaluation of angiogenesis potential of the TECs using CAM assay; (d) Comparison of the effect of molecular Dox and nanoparticle formulated AMS-6-Dox in a 2D culture of TNBC cells and in 3D TECs. Abbreviations: AOSS - acellular organ-specific scaffold; CE - chick embryo; ED - embryonic day (age of the chick embryo); iWO-DCL - immersion-agitation-assisted whole-organ DCL; DCL - decellularization; TEC - tissue engineering construct; MTT - 3-(4,5-dimethylthiazol-2-yl)-2,5-diphenyltetrazolium-bromide; CAM - chorioallantoic membrane of CE; Dox - doxorubicin; AMS-6-Dox - mesoporous silica nanoparticles loaded with $20 \%$ doxorubicin; 2D and 3D - two-dimensional TNBC cell cultures and 3D TECs.

\section{A2. Decellularization of multiple CE organs}

During the preliminary experiments, four protocols of iWO-DCL were tested by macroscopic and histological examination of the tissues, including $1 \%$ SDS, $0.1 \%$ SDS, $1 \%$ Triton-X-100 and a sequential combination of the Triton-X-100 and SDS $0.1 \%$. As a result, we found that the maximal degree of the organ-specific ECM preservation and the most efficient removal of cellular debris is achieved by the application of $0.1 \%$ SDS. The overview of the macroscopic changes of various ED18 CE organs during the iWO-DCL is shown in Figure A1. The organ-specific iWO-DCL timelines are presented in Table A1.

Table A1. Recommended protocols of iWO-DCL of ED18 chick embryo organs and tissues.

\begin{tabular}{|c|c|c|c|}
\hline Organs & Average DCL time, $h$ & Minimal washing time, $h$ & $\begin{array}{c}\text { Recommended shaking speed during } \mathrm{DCL}^{3}, \\
\text { rpm }\end{array}$ \\
\hline Brain1 & $\sim 48$ & 48 & 100 (first $24 \mathrm{~h}$ ), 90 (second $24 \mathrm{~h}$ ) \\
\hline Heart & $\sim 180-200$ & $\sim 48-60$ & 150 (first $24 \mathrm{~h}$ ), 120 (second $24 \mathrm{~h}$ ) \\
\hline Lungs & $\sim 48$ & 48 & 100 (first 24 h), 90 (second $24 \mathrm{~h}$ ) \\
\hline Liver & $\sim 240-280$ & 48 & 120 (first 5-6 days), 50 (the rest of the time) \\
\hline $\mathrm{SI}^{2}$ & $\sim 48$ & 48 & 150 (first $24 \mathrm{~h}$ ), 120 (second $24 \mathrm{~h}$ ) \\
\hline $\mathbf{P V}^{2}$ & $\sim 90-120$ & 48 & 150 (first $24 \mathrm{~h}$ ), 120 (the rest of the time) \\
\hline $\mathrm{Ve}^{2}$ & $\sim 250-280$ & $\sim 48-60$ & 150 (first $24 \mathrm{~h}$ ), 120 (the rest of the time) \\
\hline Spleen & $\sim 36-48$ & 48 & 150 (first $24 \mathrm{~h}$ ), 120 (the rest of the time) \\
\hline $\mathrm{BMu}^{2}$ & $\sim 90-120$ & 48 & 150 (first $24 \mathrm{~h}$ ), 120 (the rest of the time) \\
\hline Skin & $\sim 90-120$ & 48 & 150 (first $24 \mathrm{~h}$ ), 120 (the rest of the time) \\
\hline
\end{tabular}

${ }_{1}$ The fragmented material may be collected by centrifugation at 10,000 rpm $\times 10$ minutes.

${ }^{2}$ Abbreviations: SI - small intestine, PV - proventriculus, $\mathrm{Ve}$ - ventriculus, $\mathrm{BMu}$ - breast muscle. 
${ }^{3}$ The shaking speed has to be adjusted depending on the shaker model (radius of the orbit) in order to avoid mechanical damage of the tissues. This also may depend on the volume of the organs or tissues vs the volume of the vessel. The recommended speed is validated with the ratio of the tissues/ total volume ratio such as approximately 1:7 (e.g., $5 \mathrm{ml}$ of tissues in $35 \mathrm{ml}$ of DCL/washing solution per $50 \mathrm{~mL}$ Falcon tube). Note that the vessel should have an empty volume (in the current illustrative case it is $15-20 \mathrm{~mL}$ ) to allow free movement of the contents.

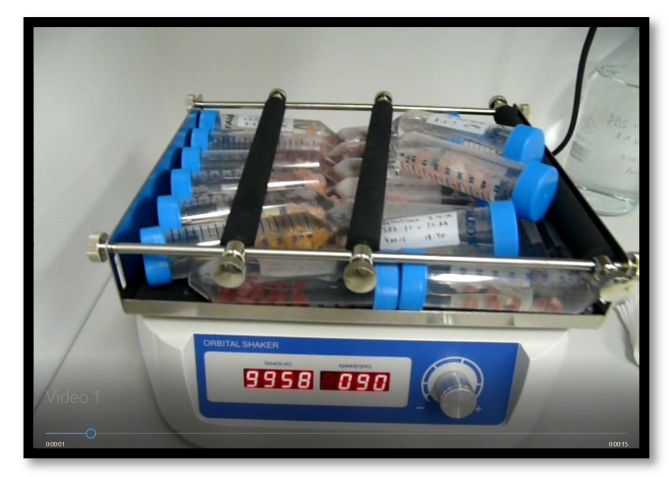

Supplementary video 1 . The screenshot of the video that demonstrates the process of iWO-DCL with the use of an orbital shaker. The tissues and organs are placed in $50 \mathrm{~mL}$ Falcon tubes with the total filled volume of the contents of $35 \mathrm{~mL}$. The shaking speed in $90 \mathrm{rpm}$. 


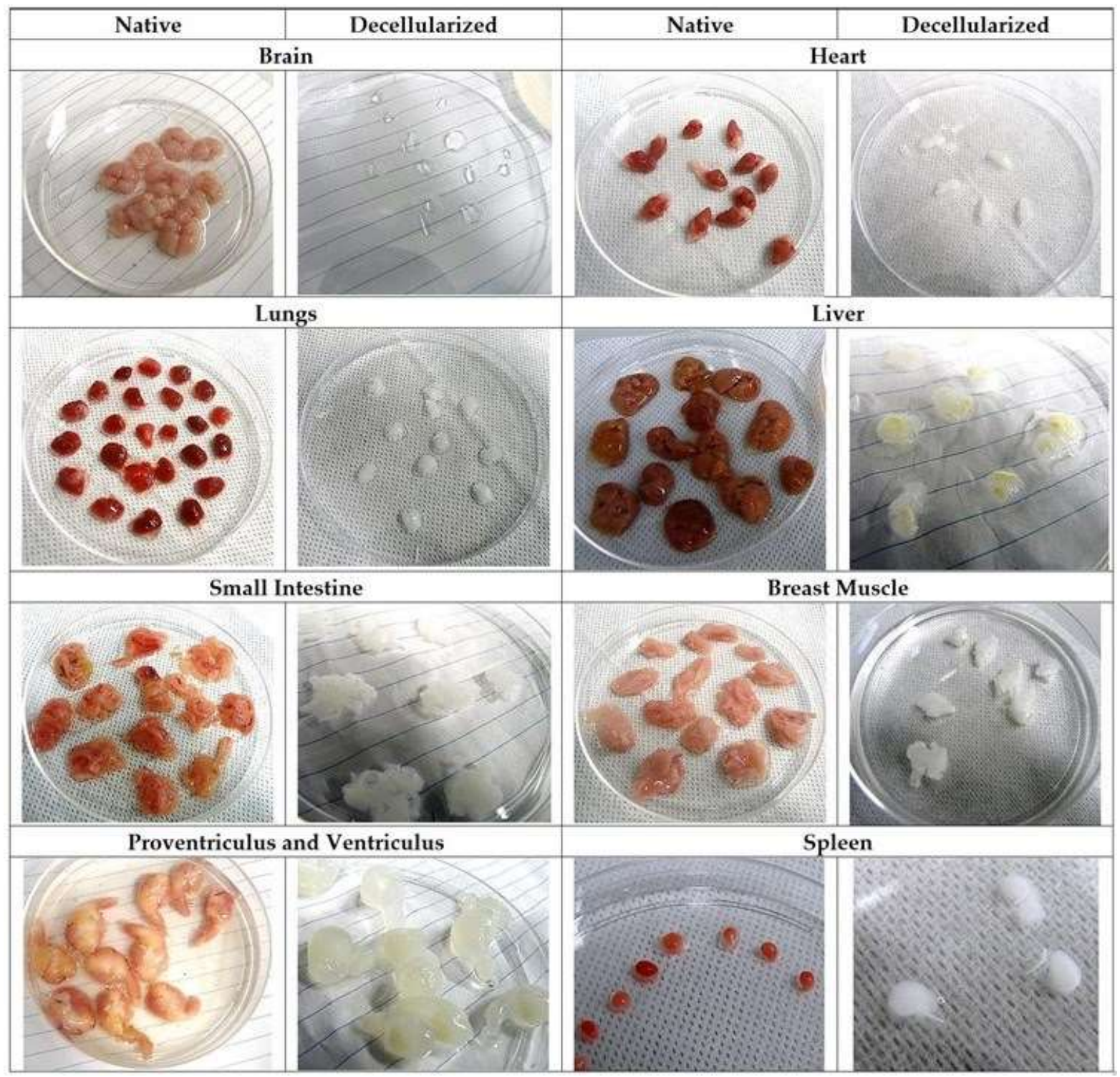

Figure A2. Effect of iWO-DCL on macroscopic appearance of ED18 CE organs at the beginning and the end of processing. Note loss of natural color, whitening of the tissue and increase of transparency. Some organs like brain, heart, lungs, liver and skeletal muscles also significantly decrease in their volume. The samples of the small intestine and spleen shown in the "Native" columns represent the organs that were processed for DCL during 4 hours to exemplify the gradual loss of the red tone starting from the edges of the organs due to the combined effects of diffusion and convection of the detergent that destroys the organ cells. This centripetal pattern of discoloration is commonly observed in the organs undergoing iWO-DCL. 
A2. Development and feasibility testing of 3D tissue engineering model of TNBC micrometastases to the liver

\section{A2.1. Native structure of chick embryo liver tissue and the effect of decellularization}

Chick embryo livers investigated in this work on ED18 had a typical hepatic histoarchitecture with vascular and biliary elements which, however, appeared a bit less distinctive than in normal human liver tissue (Figure A1 (a)). Stromal elements of the liver tissue, including central veins and portal triads) contained slightly more fibrous collagen than the hepatic parenchyma (Figure A1 (b)), while the capsular and subcapsular connective tissue structures (Figure A 1 (d)) had significant local concentration of mature fibrous collagen. The tissue was completely orthochromatic when stained by toluidine blue (Figure A1 (c)).

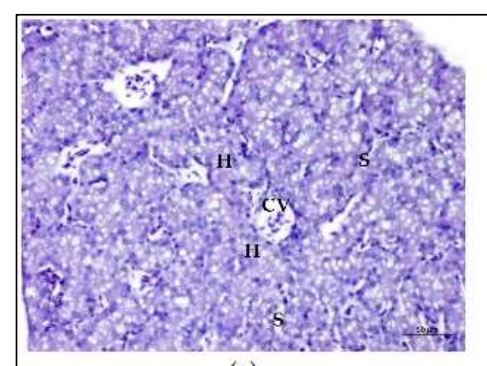

(a)

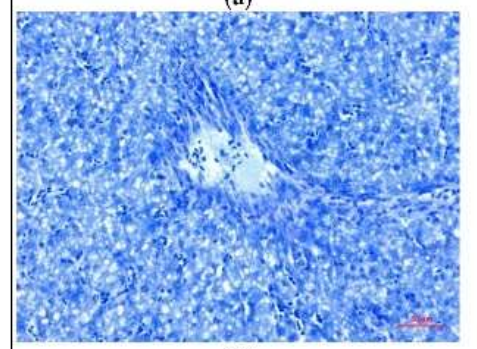

(c)

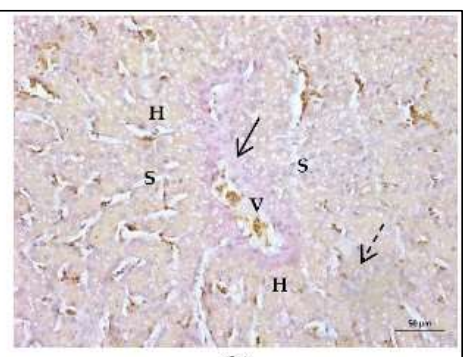

(b)

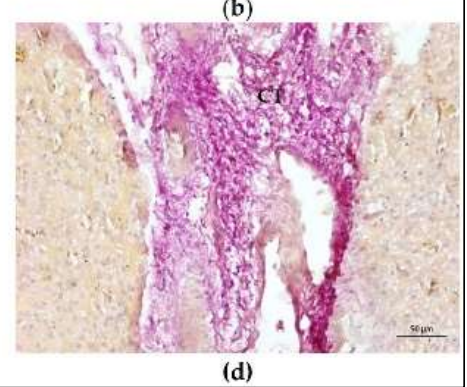

Figure A3. Histological structure of intact chick embryo liver extracted on ED18. (a) Hematoxylin and eosin staining reveals cords of hepatocytes $(\mathrm{H})$ separated by narrow voids of sinusoids $(\mathrm{S})$ which are arranged around the central vein (CV). The red blood cells in chick embryos are ellipsoid in shape and contain nuclei stained dark by hematoxylin. These cells are visible in the sinusoids and vein lumens. (b) In the samples stained by Van Gieson's method, the radial structure of hepatic lobule is more clearly discernible. Note pink staining of collagen (arrow) in the wall of a large vein (V) and more diffuse pink staining in parenchymal part of the tissue (dashed arrow). (c) Orthochromatic staining of the liver tissue by toluidine blue indicates low contents of acid glycosaminoglycans. (d) Subcapsular area of the liver. Note connective tissue (CT) of the liver capsule and interlobular septae discernible by bright fuchsinofilia in samples stained by Van Gieson's method. Strong fuchsinofilic staining reflects high concentration of mature fibrillar collagen elements. Scale bars: 50 $\mu \mathrm{m}$.

\section{A2.2. Effect of $i W O-C L$ on $C E$ liver}

The iWO-DCL procedure preserved the shape of the liver, but its volume decreased significantly and the decellularized organ became almost translucent (Figure A2). The cells and cellular debris were no longer observable in the DCL tissue by histological methods (Figure A3). The hepatic parenchyma transformed into a loose, fine mesh-like matrix formed mainly by the residuals of perisinusoidal ECM of Disse's spaces (Figure A3). The former septal connective tissue and portal elements (portal arteries and veins) and former central veins were identified in the DCL organ by their denser ECM and morphology in hematoxylin and eosin stained sections, see Figure A3 (a)) and by slightly higher collagen concentration (pink staining by Van Gieson's method, see Figure A3 (b)). After the IWODCL, two compartments of the LS-ECM became distinguishable. They are further referred 
to as the "parenchymal" compartment comprising a mesh-like matrix of the former parenchyma; and the "stromal" compartment comprising the DCL portal vasculature, interlobular septal stroma and acellular walls of former blood vessels, including central veins and portal arteries and veins (Figure A3). The effect of iWO-DCL on the separate histological elements of the liver tissue is schematically presented in Figure A4. The location of the Disse's space and the composition of the ECM of the Disse's space, according to the literature data $[79,95,96,114]$, are shown in Figure A5.

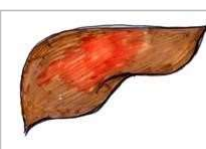

(a)

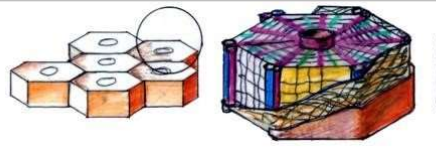

(b) (c)

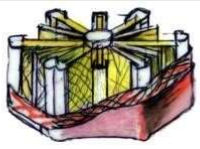

(d)

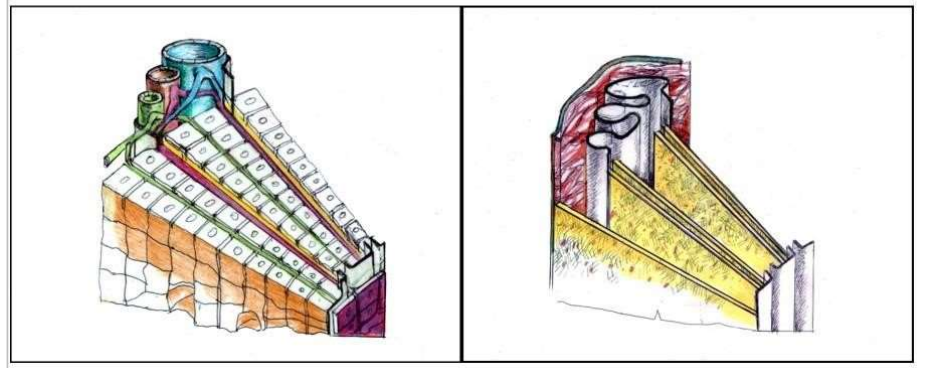

(e)

(f)
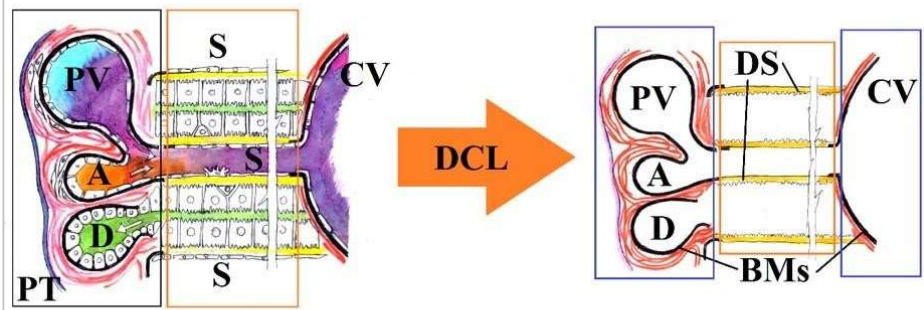

(g)

Figure A4. Schematics of the liver structure and the effect of DCL on its constituents. The liver (a) is formed by lobules (b). Each lobule (c) has a central vein in the middle and radially arranged cords of hepatocytes separated by sinusoids (radial purple lines) and bile canaliculi (radial green lines). The border of each lobule consists of loose connective tissue sheaths (red coating), which contains portal triads and interlobular vasculature branches in the corners of each lobule's "hexagon". As a result of DCL (d) all the cellular elements are removed. (e) Detailed view of the sector of a single lobule. Each cord of hepatocytes contains two rows of the polarized cells. One side of the cell row contacts with a sinusoid blood vessel (purple) through the space of Disse (yellow), while the other side of the cell row contacts a bile canaliculus (green) directly. Cells of the sinusoids and other cells of parenchyma, excepting hepatocytes, are not shown for simplicity. Blood from the portal vein (large blue blood vessel) and hepatic artery (smaller red blood vessel) is mixed in the sinusoids and transported through the liver parenchyma towards central vein in the middle of the lobule. Bile it transported to the portal bile duct in opposite direction. Portal vein, artery and bile duct make a portal triad. (f) $3 \mathrm{D}$ view of the effect of DCL on the sector of a single lobule, shown at (e). Note absence of cellular elements and preservation of collagenous stroma of interlobular septae and connective tissue sheaths of large blood vessels (red), basement membranes of large blood vessels and portal bile ducts (grey) and the ECM of Disse's space (yellow). (g) 2D detailed schematic view of a segment of a lobule before (left) and after (right) DCL and segmentation of the parenchymal and stromal compartments. Note a portal triad (PT), containing portal vein (PV), hepatic artery (A) and interlobular bile duct (D); sinusoids (S) with discontinuous endothelial lining separated from hepatocytes by Disse's space (yellow). Narrow bile canaliculi (green) are visible between the rows of hepatocytes inside the cords. Bile canaliculi do not have own cell linings. Central vein (CV) has the entire endothelial lining, basement membrane (BM) and collagenous sheath (red lines) around 
it. The structure of portal blood vessels is similar to that of the $\mathrm{CV}$, but the layer of smooth muscle cells is thicker (it exists only before DCL). Red color indicates fibrous collagen. Black lines indicate basement membranes (BM). Parenchymal compartment is shown by orange boxes, and the stromal one is indicated by blue boxes.

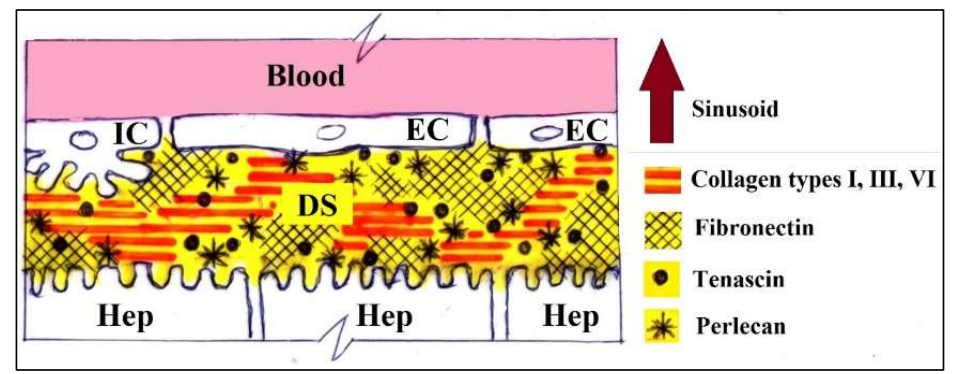

Figure A5. Schematic view of the relationship between sinusoids, Disse's space and hepatocytes (the parenchymal compartment of the liver). Note the wall of a sinusoid formed by discontinuous layer of endothelial cells (EC) and absence of basement membrane underneath the EC. The Disse's space (DS, yellow) separates the endothelial cells of sinusoids from hepatocytes (Hep) and stellate cells of Ito (IC). The concentration of fibronectin increases along the sinusoids towards the central veins. Tenascin is relatively rare component. Perlecan is predominantly associated with basement membranes. Laminin is almost absent along the sinusoids in Disse's space, excepting the area near the portal triads. The scheme is based on the information from the References $[79,95,96,114]$. 
A2.3. Structural and morphometrical analysis of $3 D T E C S$

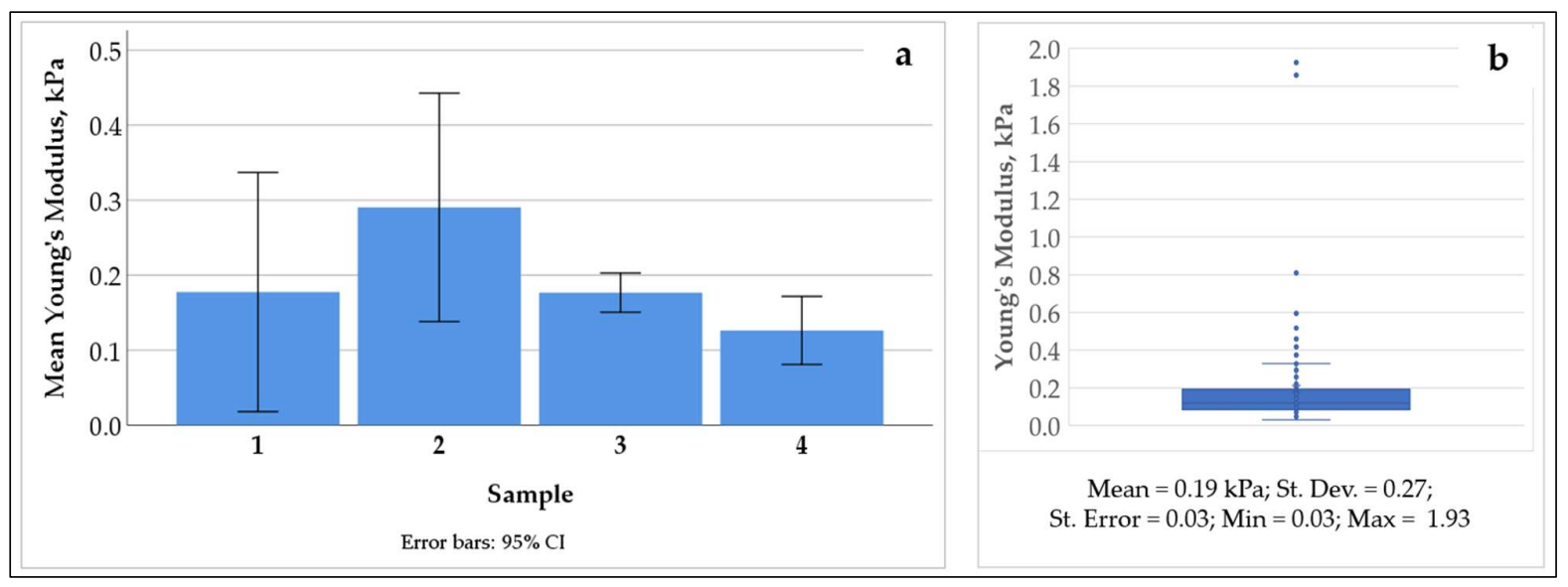

Figure A6. Mechanical characterization of the LS-ECM in hydrated state. Peak force mapping illustrates the distribution of the mean Young's modulus across four samples of CE liver AOSS (a) and overall distribution of the values in the whole set of samples (b). 


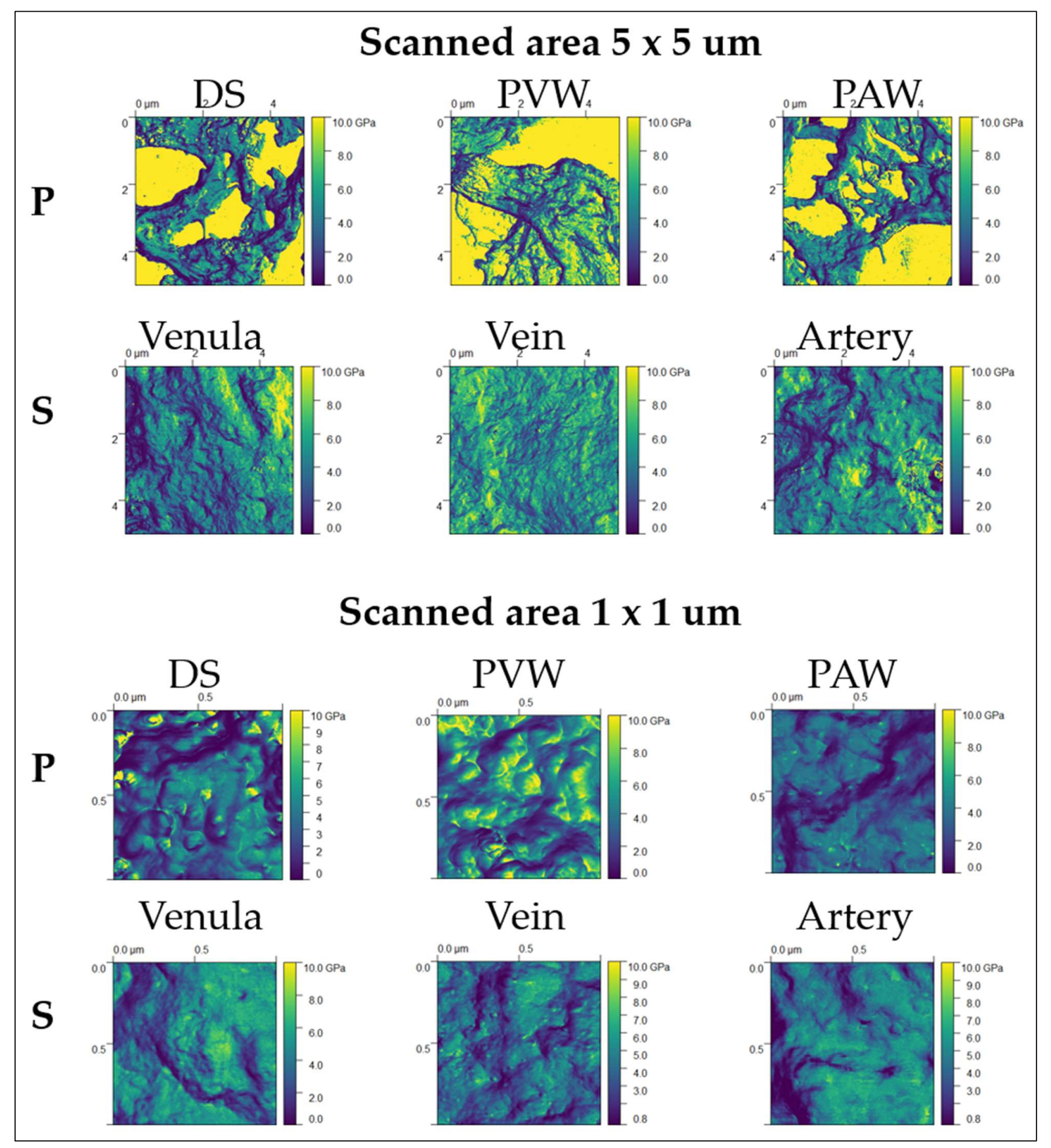

Figure A7. Stiffness mapping (normalized) of the different histoanatomical elements of the dehydrated parenchymal (P) and stromal (S) compartments of LS-ECM in CE liver AOSS measured on $5 \times 5 \mu \mathrm{m}$ and $1 \times 1 \mu \mathrm{m}$ AFM scans. Yellow tome codes higher stiffness values, deep blue tone codes the minimal stiffness (up to $2 \mathrm{GPa}$ ), and the green tone codes stiffness in the range of 2 to $8 \mathrm{GPa}$. Note that the areas colored in yellow in the $5 \times 5 \mu \mathrm{m}$ scans of the parenchymal elements corresponds with the areas without tissue (microscopy glass surface). The loose nature of parenchymal ECM allows exposure of the glass surface but results in very high variation of the stiffness values at micron scale and masking any potential quantitative differences between the histoanatomical structures and compartments. The measurements on the $1 \times 1 \mu \mathrm{m}$ scans vary much less. Abbreviations: DS - Disse's space, PVW - parenchymal ECM near vein wall, and PAW - parenchymal ECM near artery wall. 


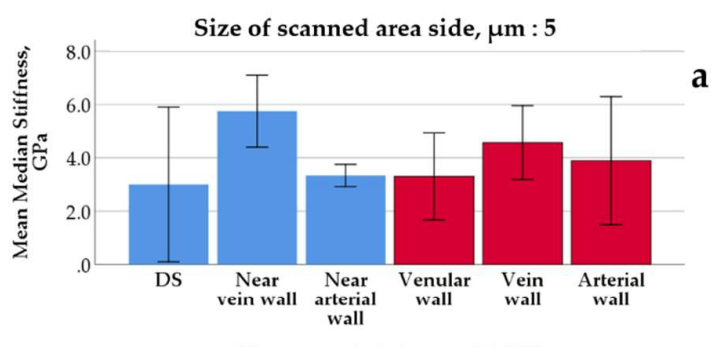

Histoanatomical element of AOSS Error bars: $95 \% \mathrm{CI}$
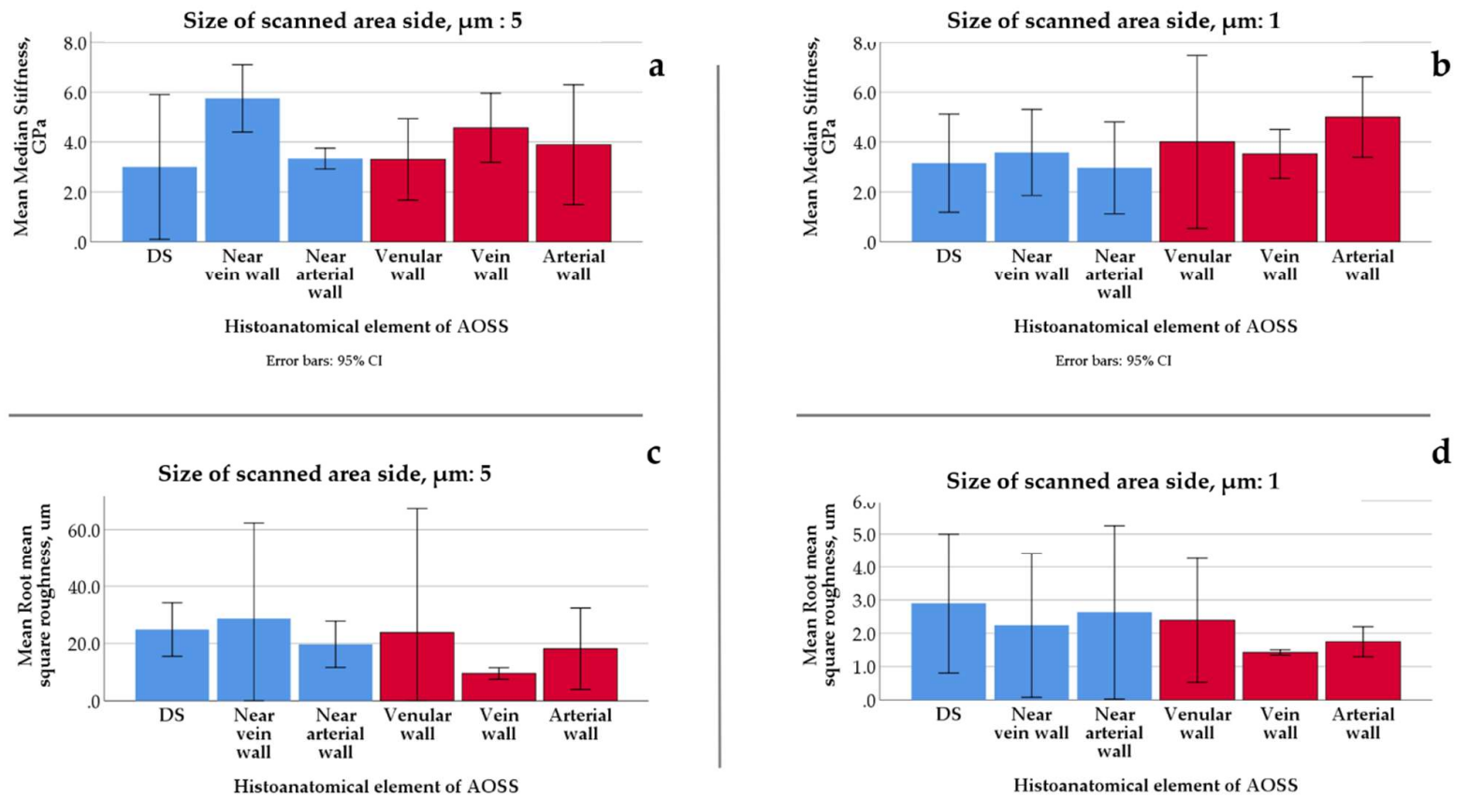

$$
\text { Error bars: } 95 \% \mathrm{Cl}
$$

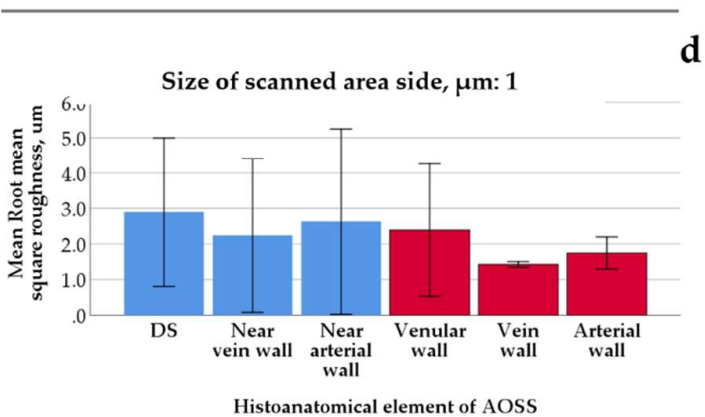

Error bars: $95 \% \mathrm{CI}$

Error bars: $95 \% \mathrm{Cl}$

Figure A8. Physical properties of the histoanatomical elements of dehydrated LS-ECM (in CE liver AOSS) measured in AFM scans with areas $5 \times 5 \mu \mathrm{m}(\mathbf{a}, \mathbf{c})$ and $1 \times 1 \mu \mathrm{m}(\mathbf{b}, \mathbf{d})$. The elements of parenchymal compartment are shown by blue bars, and the elements of the stromal compartment are shown by red bars. (a, b) Median stiffness and (c, d) root mean square roughness. No statistically significant differences of stiffness and roughness were found between the studied histoanatomical elements at both AFM imaging scales $(5 \times 5 \mu \mathrm{m}$ and $1 \times 1 \mu \mathrm{m})$ in the given set of samples $(\mathrm{N}=18$ for each scanned area size). Abbreviations: DS - Disse's space, "Near vein wall" and "Near arterial wall" means the properties of the areas of the parenchymal compartment measured in vicinity to veins and arteries. 


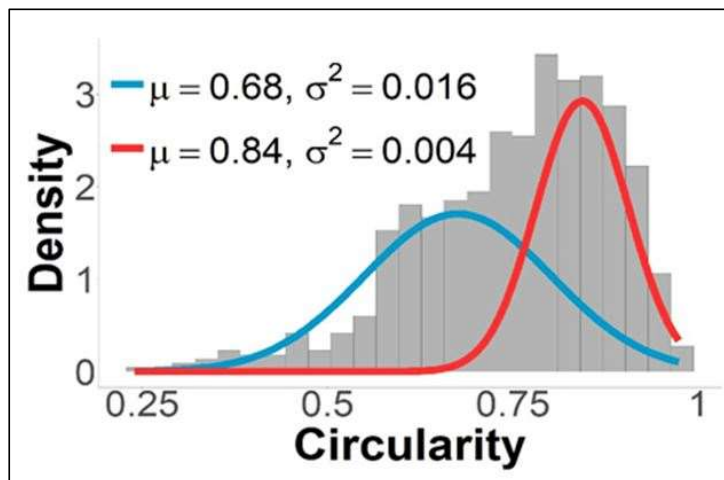

(a)

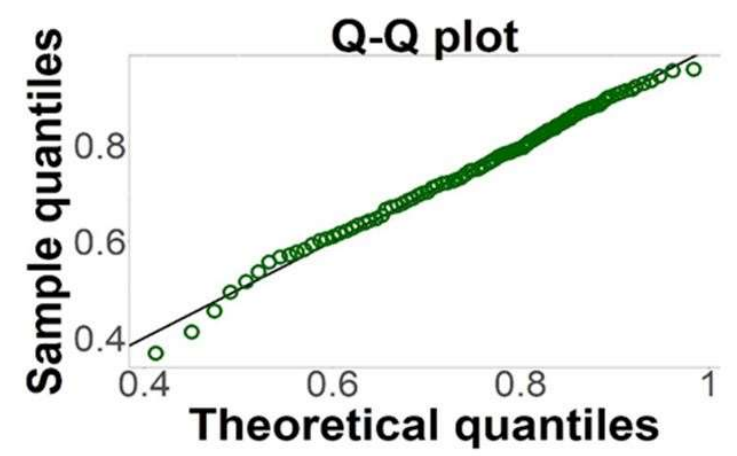

(b)

Figure A9. (a) A histogram of circularity values of the total number of 706 individual cells examined in TECs collected after 1 week of in vitro culture. The data is best described by two normal distributions (blue and red curves represent probability density functions) and reveals formation of two subpopulations of cancer cells with different circularities such as elongated oval-shaped "mesenchymal-like" and nearly circular-shaped "epithelioid" cells with circularity values $\sim 0.68$ and $\sim 0.84$, respectively. (b) Comparison between the quantiles of the theoretical bimodal normal distribution with the quantiles of the observed data. The data points are positioned close to $y=x$, indicating that the distribution of the sample data is similar to the normal distribution.

Table A2. Relative cellularity (Mean $\% \pm$ Standard Deviation of the TEC section area) in different compartments of the liver ECM measured as a ratio of cells to matrix area: results of image analysis of histological sections. 10 images were analyzed for each timepoint/compartment.

\begin{tabular}{cccc}
\hline \multirow{2}{*}{ Sampling time point } & & Compartment & \\
\cline { 2 - 4 } & Parenchymal & Stromal & Mixed \\
\hline Week 1 & $5.1( \pm 6.9)$ & $16.3( \pm 15.2)$ & - \\
\hline Week 2 & $6.8( \pm 6.5)$ & $17.3( \pm 9.4)$ & $5.5( \pm 3.4)$ \\
\hline Week 3 & $10.0( \pm 3.0)$ & $37.7( \pm 21.0)$ & $10.6( \pm 7.8)$ \\
\hline Week 4 & $1.8( \pm 1.4)$ & $1.2( \pm \mathrm{NA})$ & $5.6( \pm \mathrm{NA})$ \\
\hline
\end{tabular}


Table A3. Estimated values of the logistic growth model parameters with confidence intervals (CI95\%).

\begin{tabular}{|c|c|c|c|c|}
\hline \multirow[t]{2}{*}{ Parameters } & \multicolumn{2}{|c|}{$2 \mathrm{D}$} & \multicolumn{2}{|c|}{$3 \mathrm{D}$} \\
\hline & Estimated value & $\mathrm{CI}_{95 \%}$ & Estimated value & $\mathrm{CI}_{95 \%}$ \\
\hline $\mathrm{C}_{0}$ & 0.03181 & $-0.2501,0.3137$ & 0.006906 & $-0.01293,0.02675$ \\
\hline $\mathrm{C}_{\max }$ & 0.8891 & $0.6392,1.139$ & 0.1524 & $0.1107,0.1941$ \\
\hline $\mathrm{d}$ & 0.6325 & $-0.7616,2.027$ & 0.2826 & $-0.005646,0.5708$ \\
\hline \multicolumn{2}{|c|}{ Characteristics of fitting } & $2 \mathrm{D}$ & & $3 \mathrm{D}$ \\
\hline \multicolumn{2}{|c|}{ SSE } & 0.01974 & \multicolumn{2}{|r|}{0.0002222} \\
\hline \multicolumn{2}{|c|}{ R-square } & 0.9634 & \multicolumn{2}{|r|}{0.9864} \\
\hline \multicolumn{2}{|c|}{ Adjusted R-square } & 0.9267 & \multicolumn{2}{|r|}{0.9729} \\
\hline \multicolumn{2}{|c|}{ RMSE } & 0.09935 & \multicolumn{2}{|r|}{0.01054} \\
\hline
\end{tabular}



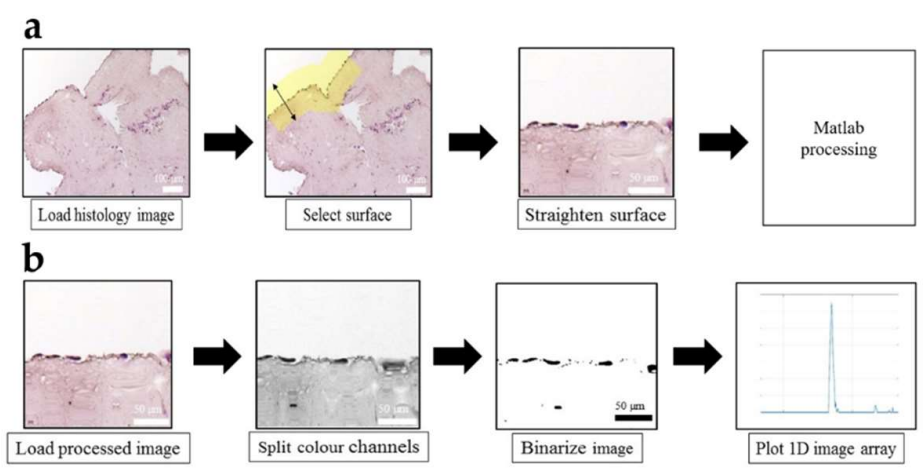

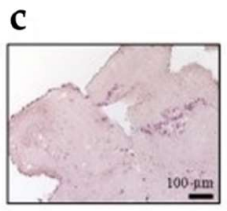

d Day 5

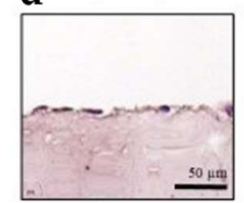

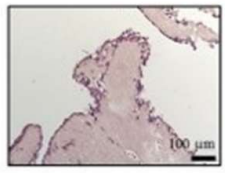

Day 13

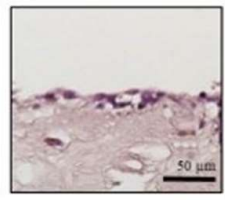

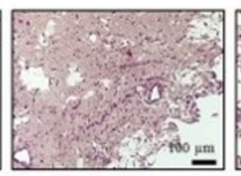

Day 21

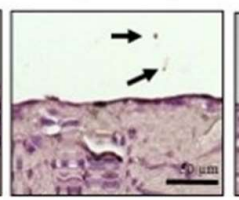

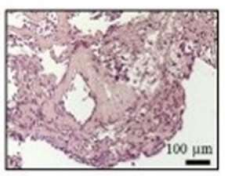

Day 28

Figure A10. (a, b) The procedure of the digital image analysis of surface colonization pattern illustrated with a histology image of the CE liver / TNBC TEC at day 5 of in vitro culture. (a) The first part of the algorithm is implemented in ImageJ and includes loading of image, selection of the surface area of 300 pixels in width and straightening of the selected surface. The arrow on the yellow shade indicates the width of 300 pixels. (b) The second part of the algorithm is implemented in Matlab and includes loading of the image processed in ImageJ, splitting of color channels, binarization of the green channel image with the with a threshold of 0.35 and creation of 1D image array, e.g. the image's signal intensity is plotted versus the matrix depth in $\mu \mathrm{m}$. (c, d) Distribution of the cells near the surface of the TEC over a period of 28 days. (c) Original histology images and (d) corresponding images of the digitally straightened and analysed surface areas of the TECs. Arrows indicate sample impurities which may occur during sample preparation. (e, $g, f)$ Results of the digital image analysis of the TECs' surfaces colonization patterns. Note the linear growth of cellular density near the TECs surfaces over the observation time. (e) Signal intensity in the surface areas of the TECs over the time (28 days). (f) Average number of cells per $200 \mu \mathrm{m}^{2}$ in the surface areas of the TECs over time (28 days). (g) Average number of cells versus signal intensity (pixel) at four different time points $(5,13,21$ and 28 days). 


\section{A2.4. Angiogenic assay on chick embryo CAM}

\section{A2.4.1. Egg preparation and grafting procedure for angiogenic analysis}

The experiment was approved by the Animal Ethics Committee of Macquarie University (ARA 2015/006). The fertilized White Leghorn chicken eggs were incubated for 72 $\mathrm{h}$, until embryonic day 3 (ED3). Then the eggs were placed in a horizontal position and left in the incubator for the next $30 \mathrm{~min}$ for repositioning of the embryos. Afterwards, the tops of the blunt ends of the shells were wiped with $70 \%$ ethanol, and 3-4 $\mathrm{mL}$ of the egg white was extracted from the bottom part of the egg by puncturing of the blunt end of the shell with a syringe needle (18G) at the angle of $\sim 45^{\circ}$ (Figure A17, Day 3). The albumin extraction resulted in decrease of the total volume of the egg and dropping of the chorioallantoic membrane (CAM), which were necessary for the further grafting procedure. After the extraction, the stab holes were sealed with a sticky tape and the eggs were returned to the incubator until ED8. On ED8 the eggshells were cut on the blunt end to create a lid and expose the CAM. Following this, the grafting procedure was performed (Figure A17, Day 8).

All the embryos were divided into 4 groups, with 10 eggs per a group. The group 1 was used as a control (labelled "Control") and left ungrafted in order to evaluate the parameters of natural angiogenesis occurred in chick embryos during the period between ED8 and ED12. In other groups the following materials were aseptically implanted onto CAM under sterile conditions: 1) chick embryo liver AOSS soaked in complete culture media following the pre-seeding protocol (labelled "Scaffold") for $24 \mathrm{~h}$; 2 ) cell suspension of MDA-MB-231 cells, $2 \times 105$ cells in $60 \mu \mathrm{L}$ of complete culture media (labelled "Cells") and 3) the TECs, prepared as described above and cultured in vitro for 12 days prior grafting (labelled "TEC"). This time period of preliminary culture of TECs in vitro was chosen to obtain the engineered tumor samples containing the similar number of cells $\left(\sim 2 \times 10^{5}\right.$ cells per sample) in the compared TECs and cell suspension xenografts. This period was also preferred to obtain the TECs with actively grown cell populations.

Following this, the images of the CAM were taken with a stereomicroscope as described in the section A6.2 "Imaging of CAM in vivo" below. Then the eggs were sealed and returned back to the incubator and maintained under standard conditions with excluded rotation. Afterwards, on ED12, the eggshells were re-opened, and the imaging was repeated under the same conditions as on ED8. After the imaging session on ED12 the chick embryos were euthanized by quick decapitation. The CAM with grafter materials and control samples of ungrafted CAM were dissected, washed in PBS and studied by histological methods.
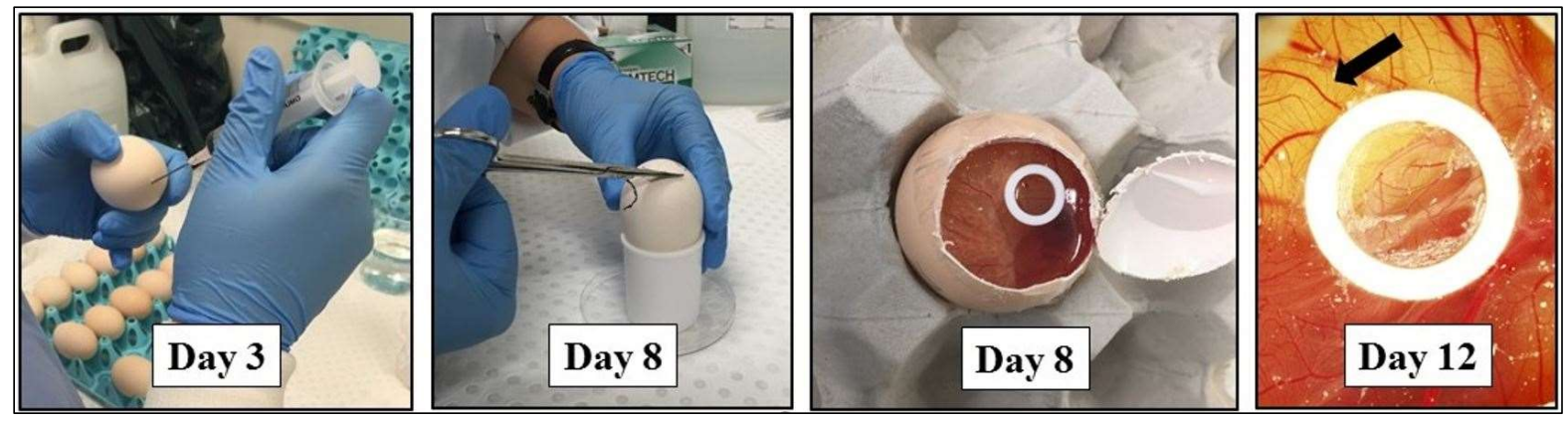

Figure A11. Illustration of grafting protocol. The following sequential steps are shown: Extraction of the egg white on ED3. Opening of the egg on ED8. Grafting of the tested samples on the CAM on ED8. The cell suspension grafting is shown; a sterile white rubber ring taken from a $1 \mathrm{~mL}$ cryovial is used to prevent the leakage of the suspension. The last (right) image shows the same egg on ED12. The arrow shows a bifurcation of a blood vessel as a preferential grafting site. 


\section{A2.4.2. Imaging of CAM in vivo}

The CAM of the eggs with open shell lids were imaged in vivo using the Olympus MVX10 (Olympus, Japan) stereomicroscope equipped with an eyepiece magnification ranging between $0.63 \times$ to $6.3 \times$, a fixed focus and two objective lenses, $1 \times / 0.25$ N.A. and $2 \times / 0.5$ N.A. The microscope featured long working distance $(20$ to $87 \mathrm{~mm}$ ), adjustable field-of-view (FOV, 1.7 to $55 \mathrm{~mm}$ in diameter). To maintain the healthy state of the chick embryos the heating stage ThermoPlate by Tokai Hit (Shizuoka-ken, Japan) was used during in vivo inspection of CAM. The objective $1 \times / 0.25$ N.A. was applied for the rough focusing and the lens magnification of $2 \times$ was used for the detailed imaging. The samples were illuminated from above by a $100 \mathrm{~W}$ mercury lamp. In order to achieve a higher contrast of the red color of the blood, a filter cube with blue illumination light was used. This filter cube contained one single-edge short pass dichroic beam splitter (Semrock, USA) with transmission of the wavelengths above $750 \mathrm{~nm}$ and a single-band bandpass (510570 $\mathrm{nm}$ ) filter as the emission filter by (Semrock, USA). The microscope was coupled with a motorized Z focus ProZ Stand (Prior Scientific, USA), which provided seamless zooming from $40 \times$ to $1250 \times$ magnification (used for the focusing and study purposes).

\section{A2.4.3. Quantification of angiogenesis}

We evaluated the morphological parameters of angiogenesis by determining quantitative features of newly developed vessels within CAMs on ED8 and ED12. We analyzed images of five eggs for each experimental group (intact eggs, cell suspensions, scaffolds, and TECs). In particular, we quantified the density (number per unit area) and length of major blood vessel sections between the branching points (segments), the blood vessels of the first order (extremities) [115] and the density of branching points [49] (Figure A18). In this approach a branch from one major vessel creates two new vessels; also, the end of a vessel is counted as a branching point. Other angiogenesis parameters are calculated as described elsewhere [115] and [49].

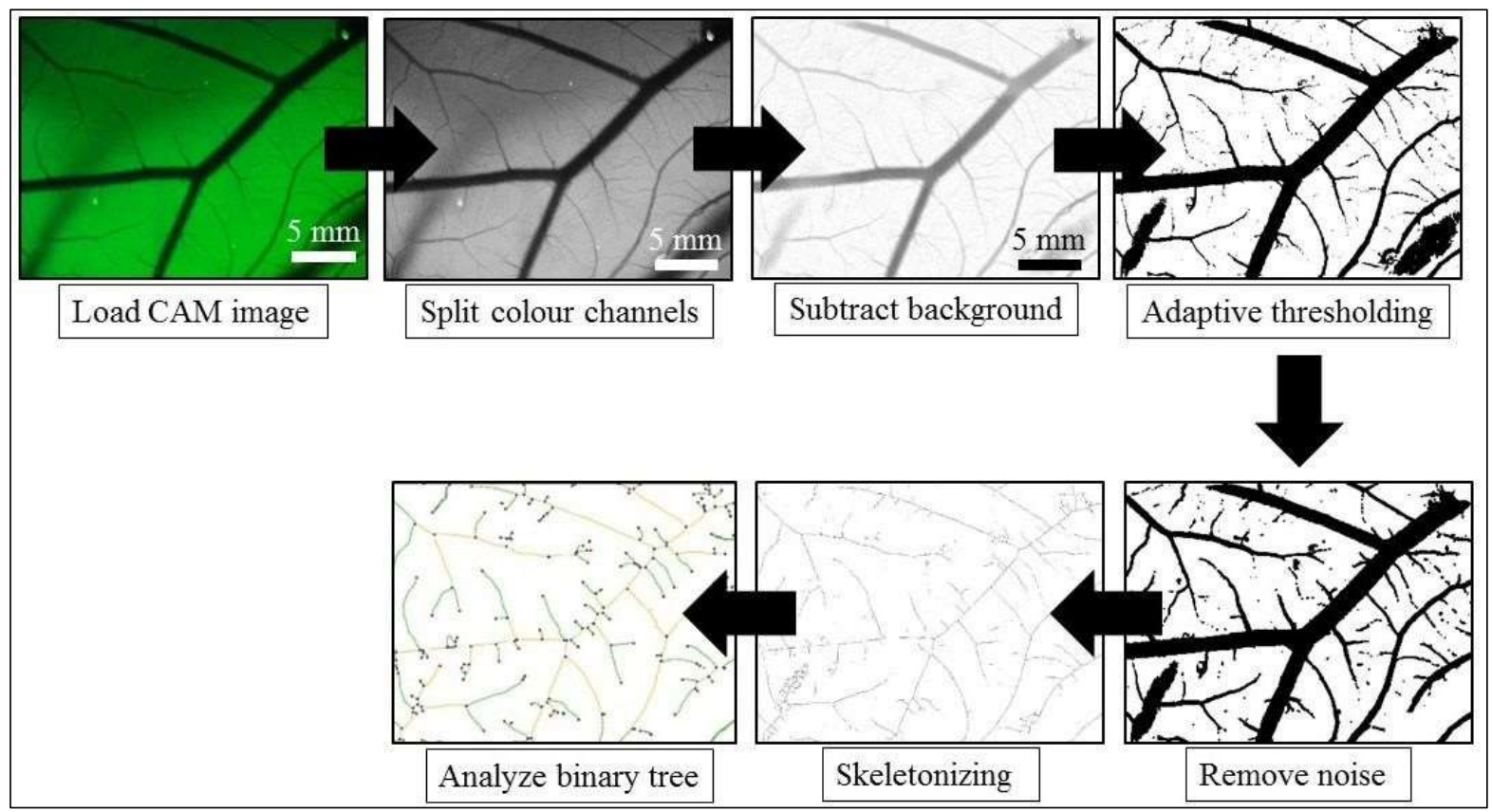

Figure A12. Algorithm used for the validation of induced blood vessel growth. Angiogenesis Analyzer was applied to skeletonized images. Red dots in the final panel represent the branching points whereas the green lines represent the vessels of first order (extremities) and the yellow lines show the vessels of second order as defined in [115]. 
The main steps of the image analysis algorithm are the subtraction of the background of the image, the adaptive thresholding, noise reduction and the skeletonization as described in References [116] and [115]. To reduce the noise erosion and dilation transformation were applied individually on each image.

After skeletonizing, the ImageJ plugin Angiogenesis Analyzer [51] was applied to each egg, four ROIs were selected, representing four opposite sides of the graft. The plugin estimates the number of branching points and total vessel length, whereas the vessels are divided into different elements. The detailed description and illustration of terminology used for quantification of binary tree of blood vessels structure can be found in the ImageJ documentation [51]. Statistical significance of the differences between the studied groups' parameters of blood vessel trees was analyzed with use of one sample Kruskal-Wallace test, followed by comparisons in the pairs of the groups with use of U-test by Mann-Whitney.

The results (Figure A19) show a statistically significant increase of the following parameters per unit area in TECs on ED12 in comparison to intact controls (normal embryonic angiogenesis, ED12): the total blood vessel length, or vascular density ( $p=0.07$ ) (Figure A19 (a)); the number of junctions ( $p=0.020)$ (Figure A19 (b)); the length of segments (the parts of the blood vessels tree between enclosed 2 neighbor branching points) $(p=0.003)$ (Figure A19 (c)); the number of first order vessels (extremities) $(p=0.014)$ (Figure A19 (d)); the number of branches ( $p=0.039)$ (Figure A19 (e)); and statistically non-significant increase in the length of branches $(p=0.121)$. Statistically significant increase of the number of branching points per area $(p=0.020)$ is illustrated in the main text of the paper (see Figure $8(\mathrm{~g})$ ). Unseeded scaffolds and cancer cell suspensions were found not to affect normal embryonic angiogenesis as there were no statistically significant differences between the scaffolds and intact controls.

Also, there were no statistically significant differences in the parameters of the blood vessel tree architecture measured on ED8 between the studied groups (data not shown). 


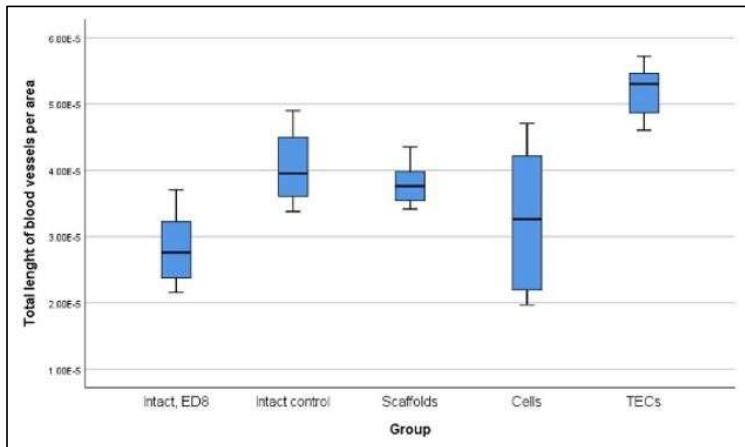

(a)

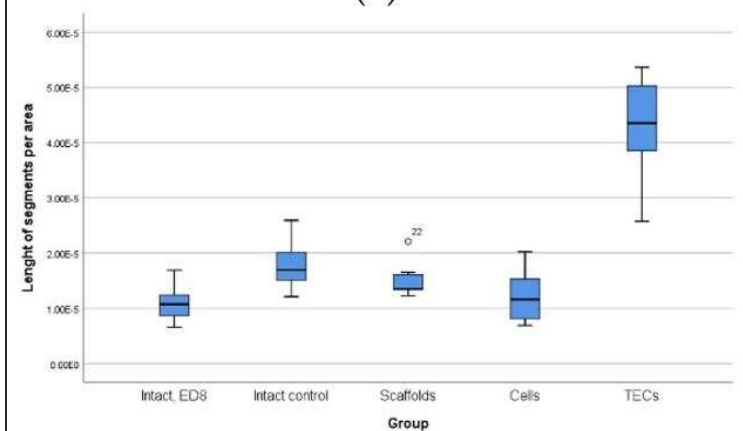

(c)

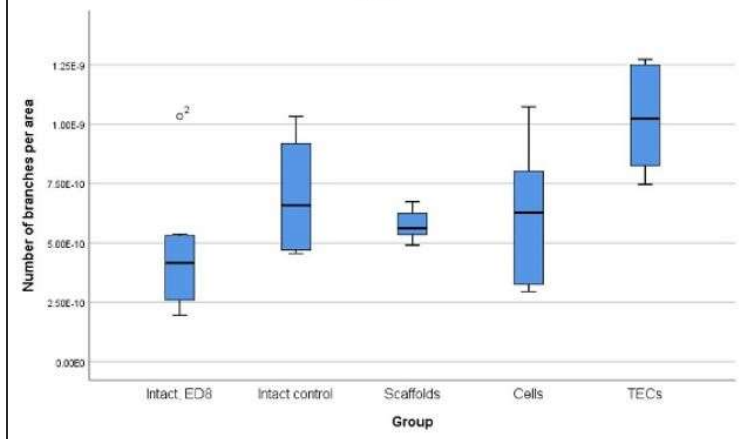

(e)

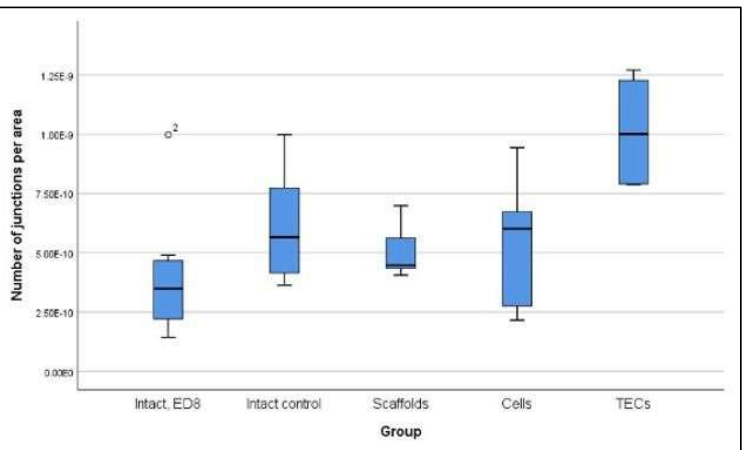

(b)

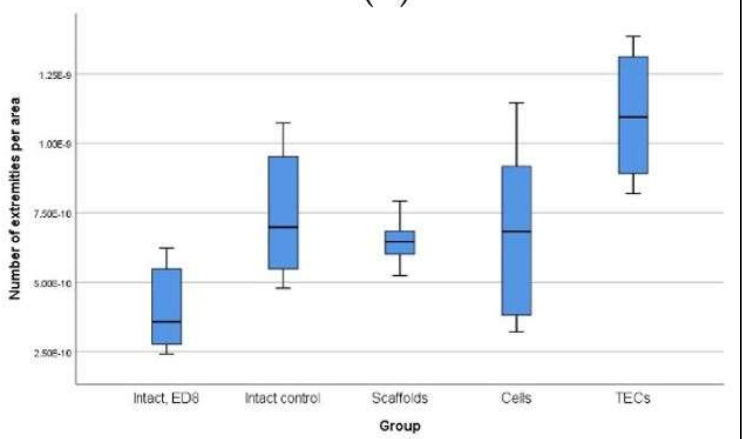

(d)

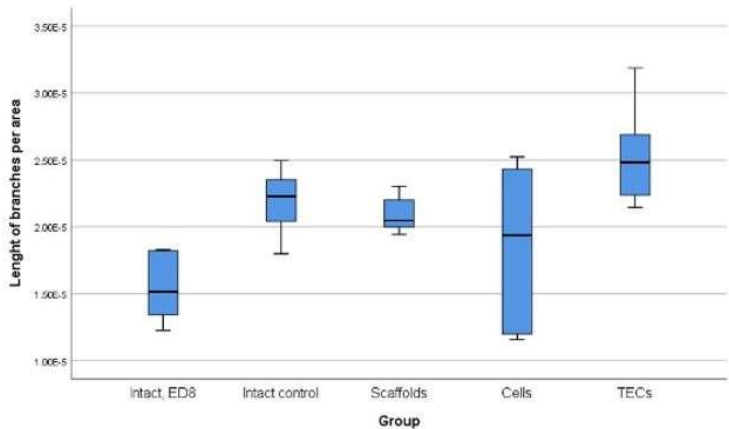

(f)

Figure A13. Quantitative analysis of angiogenesis. The parameters of the blood vessel tree are reflected as values per unit area. (a) Total blood vessels' density; (b) Number of Junctions; (c) Length of segments; (d) Number of extremities; (e) Number of branches; (f) Length of branches. The results are presented as boxplots, where thick dark horizontal line shows mean values, blue boxes indicate C195\% for mean and the whiskers indicate minimal and maximal observed values. 


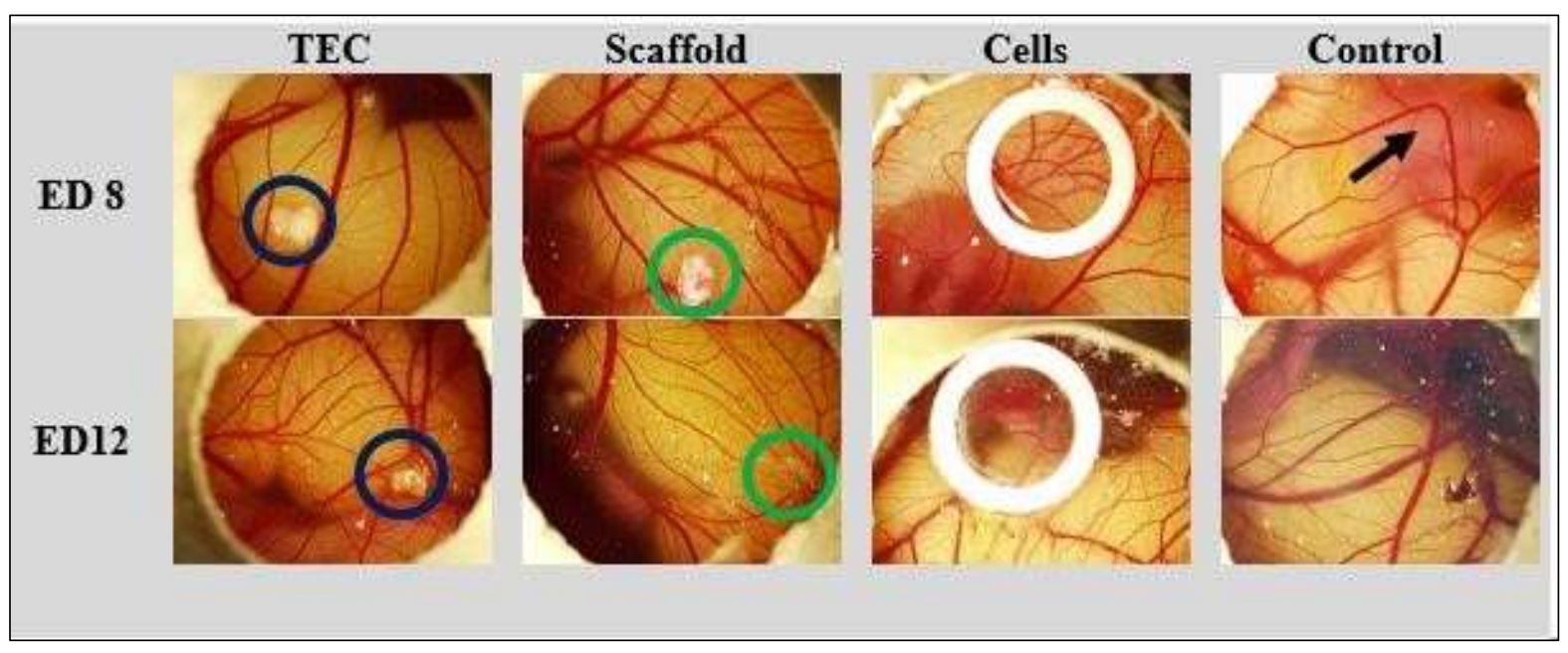

Figure A14. Architecture of CAM blood vessels in studied groups. Low-magnification $(\times 0.63)$ view of control and grafted CAM on ED8 and ED12 obtained using bright-field microscopy. Angiogenic effects induced by the following chick embryo CAM grafts: 3D TEC (TEC, labelled with blue circles), liver AOSSes (Scaffold; labelled with green circles), and suspension of MDAMB-231 cells (Cells; xenograft suspension was placed into the chamber formed by a white rubber ring), in comparison with natural embryonic development of CAM vasculature (Control) during a period between ED8 and ED12. Note that the degree of vascularization of CAM on ED8 was naturally variable between embryos. Representative images of the same eggs on ED8 and ED12 were used for analysis and shown for each group. Equal volume samples of the grafted TECs and AOSSes were used. The number of cells $(2 \times 105)$ in the cellular xenograft and TECs were approximately equal. An arrow in Control CAM, ED8, indicates the chick embryo residing underneath the CAM. White dust on the surface of CAM is small fragments of the eggshell dropped on the CAM during the shell windowing. Note blood vessels converging towards the grafts in TECs and Scaffolds groups. 
A2.5. 3D TECS of TNBC micrometastases to the liver as drug and nanomedicine testing platform A2.5.1. Characterization of nanoparticles

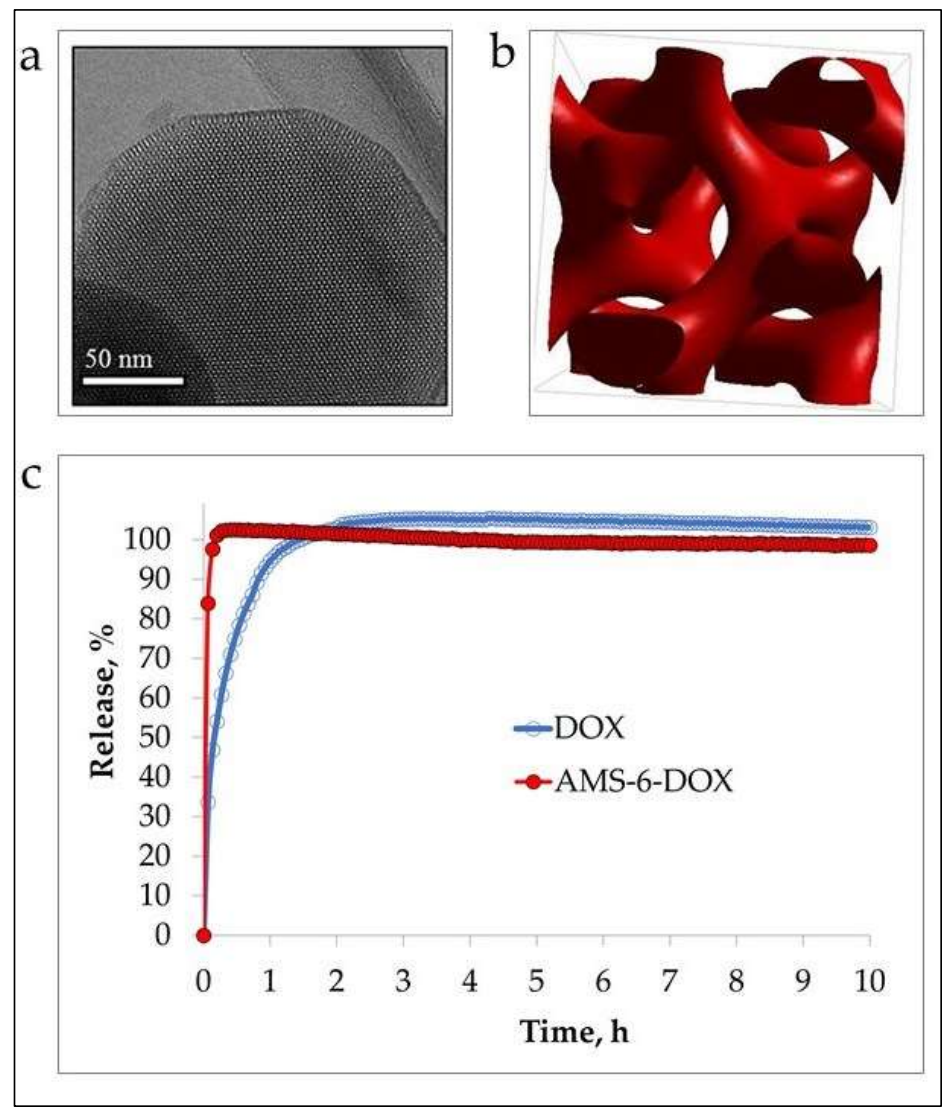

Figure A15. (a) Typical TEM images of calcined AMS-6 mesoporous material showing a high degree of mesoporous order and (b) Schematics of the of AMS-6 structure. (c) Kinetics of Dox release from AMS-6-Dox vs the free Dox drug dissolution in PBS.

Table A5. Zetapotential of AMS-6 and AMS-6-Dox nanoparticles.

\begin{tabular}{ccccc}
\hline Sample & Temperature, ${ }^{\circ} \mathrm{C}$ & $\mathbf{p H}$ & $\begin{array}{c}\text { Polydispersity } \\
\text { index ((Dw/Dm) })^{2}\end{array}$ & Zeta-potential, $\mathbf{m V}$ \\
\hline Pure AMS-6 in PBS & 25 & 7.2 & 0.544 & -10.9 \\
\hline Pure AMS-6 in CCM & 25 & 7.4 & 0.685 & -8.3 \\
\hline AMS-6-Dox in PBS & 25 & 7.2 & 1.000 & -11.2 \\
\hline AMS-6-Dox in CCM & 25 & 7.4 & 1.000 & -8.8 \\
\hline
\end{tabular}




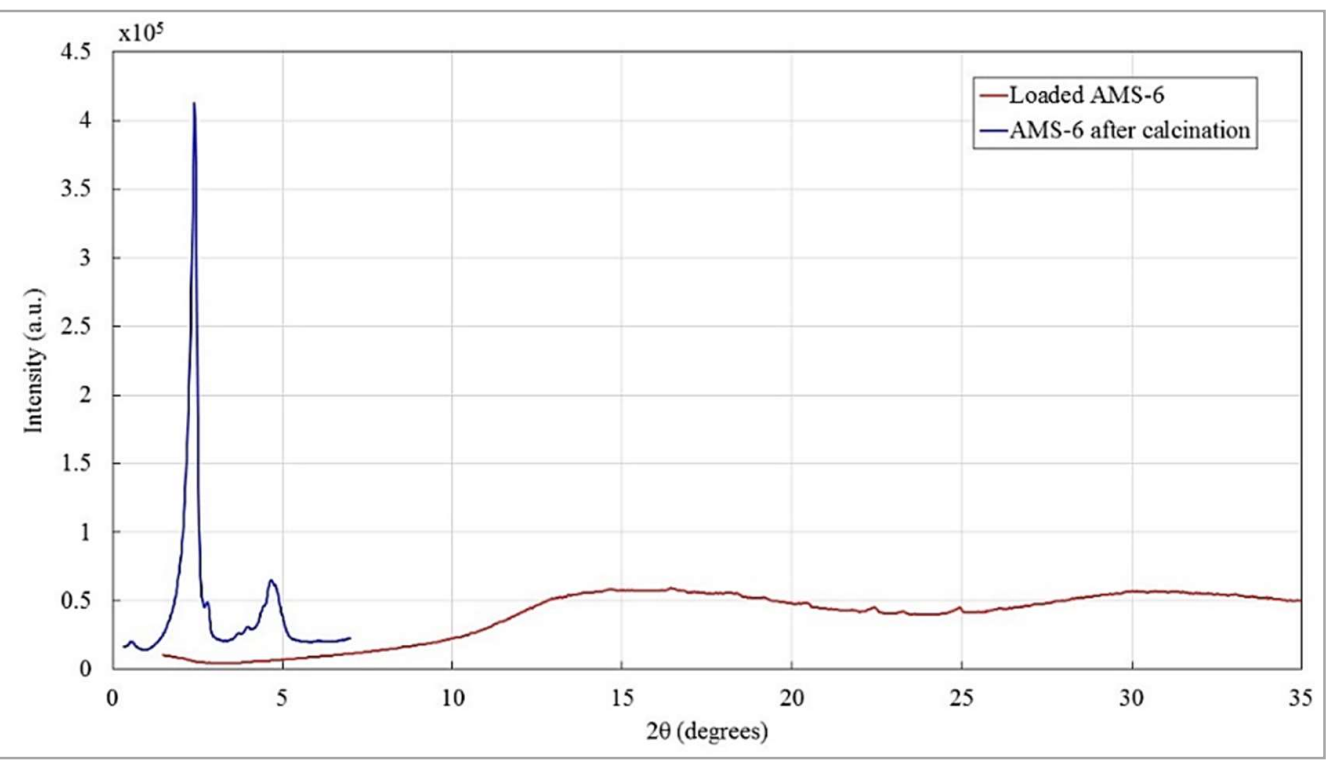

Figure A16. X-ray diffraction (XRD) pattern of pure calcined AMS-6 mesoporous nanoparticles (blue) and the loaded AMS-6-Dox nanoparticles (red). The measurements of the pure calcined AMS-6 NPs reveal a highly ordered mesostructured with peaks of scatter in-between the pores of the nanoparticles. The typical XRD pattern of the pure sample display peaks at low angles of 2.4 and $4.8^{\circ}$ with an intensity of $4.12 \cdot 10^{5}$ and $0.6 \cdot 10^{5}$ a.u., respectively. The loaded AMS-6-Dox sample curve shows small peaks at high angles of approximately 15 and $30^{\circ}$ with intensities below $0.75 \cdot 10^{5}$ a.u., which are most likely the scattering patterns of crystallized Dox outside of the pores. The pores of the AMS-6-Dox still seem to be filled with DOX since the pattern does not show any peaks at low angles which would indicate scatterings between the pore walls of the nanoparticles.

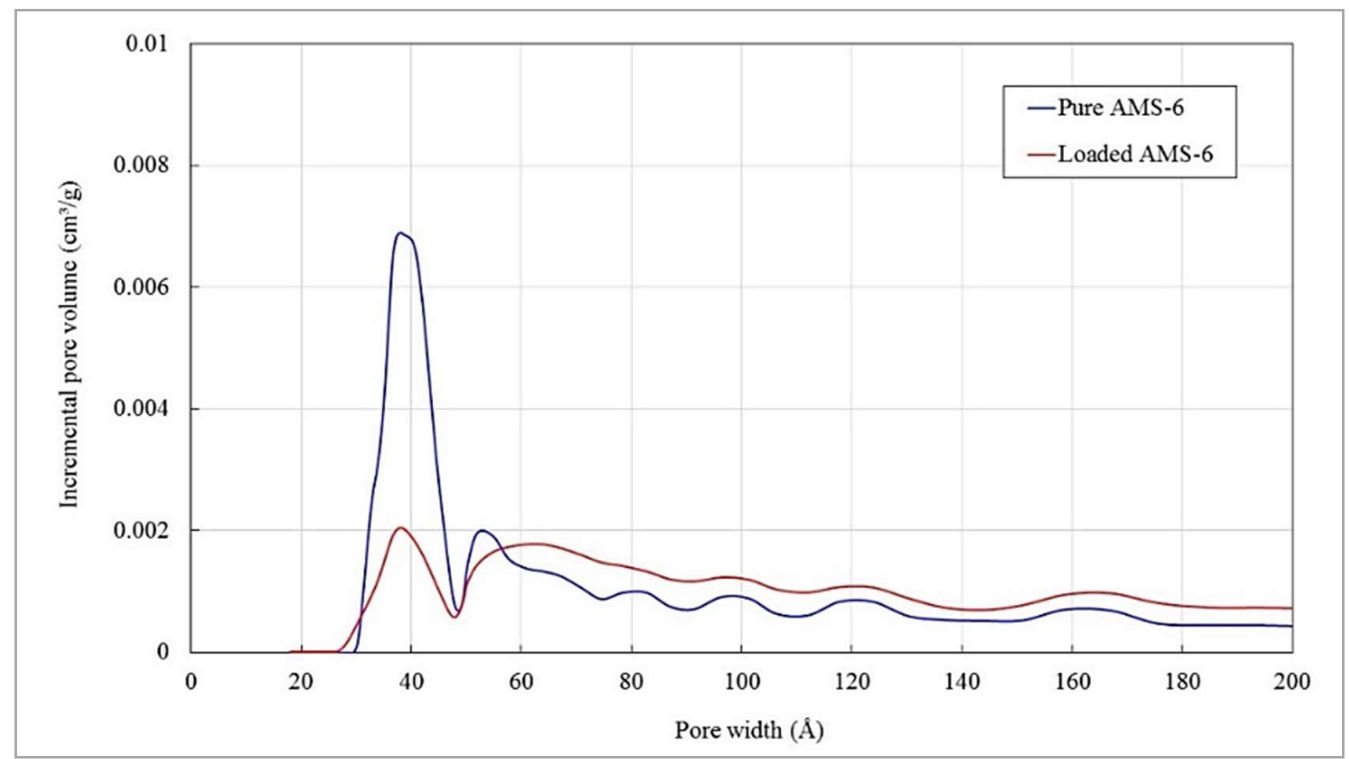

Figure A17. DFT pore size analysis. The pore width of pure AMS-6 and loaded AMS-6-Dox nanoparticles is in a range of 30 to $50 \AA$. 


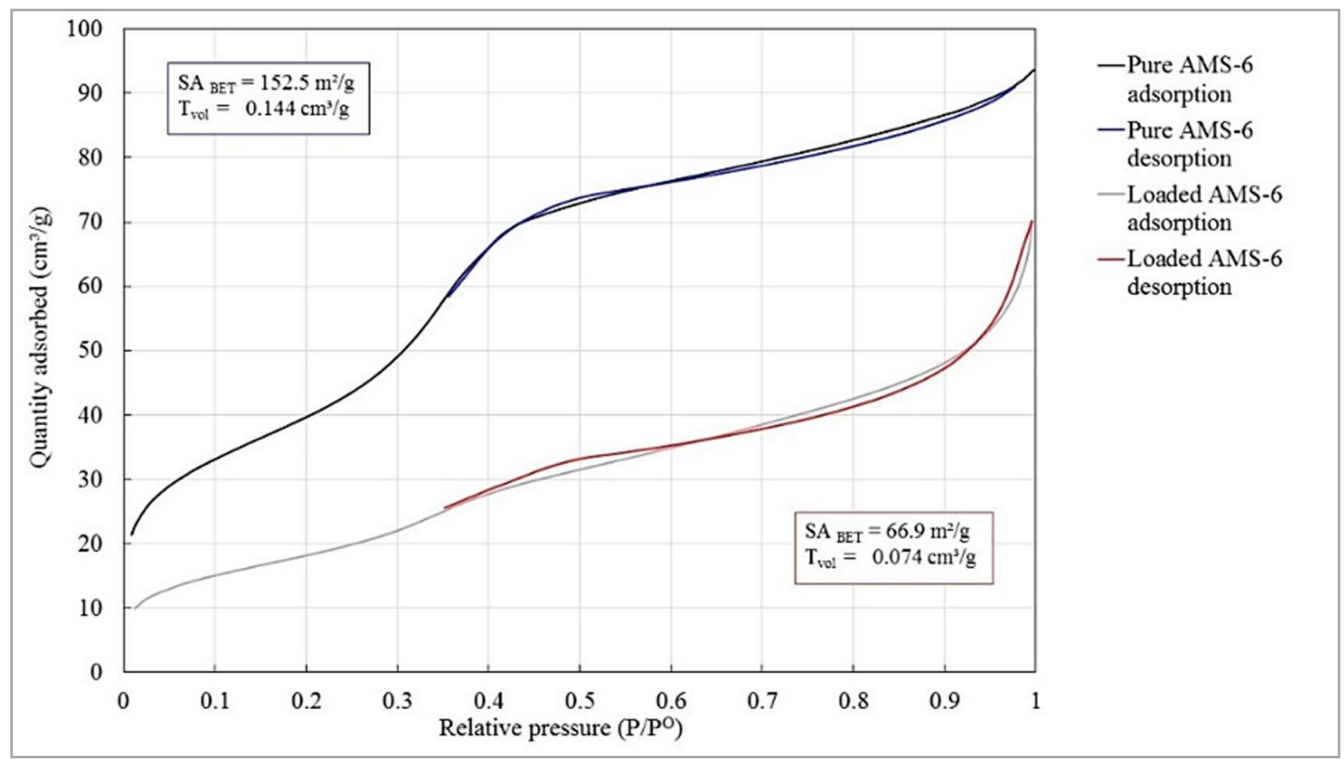

Figure A18. Nitrogen adsorption/desorption isotherm curves of pure AMS-6 and loaded AMS-6-Dox nanoparticles. A very narrow hysteresis loop where the plotted adsorption and desorption curves are close together results from the fact that the pore width of $3.81 \mathrm{~nm}$ is very small.

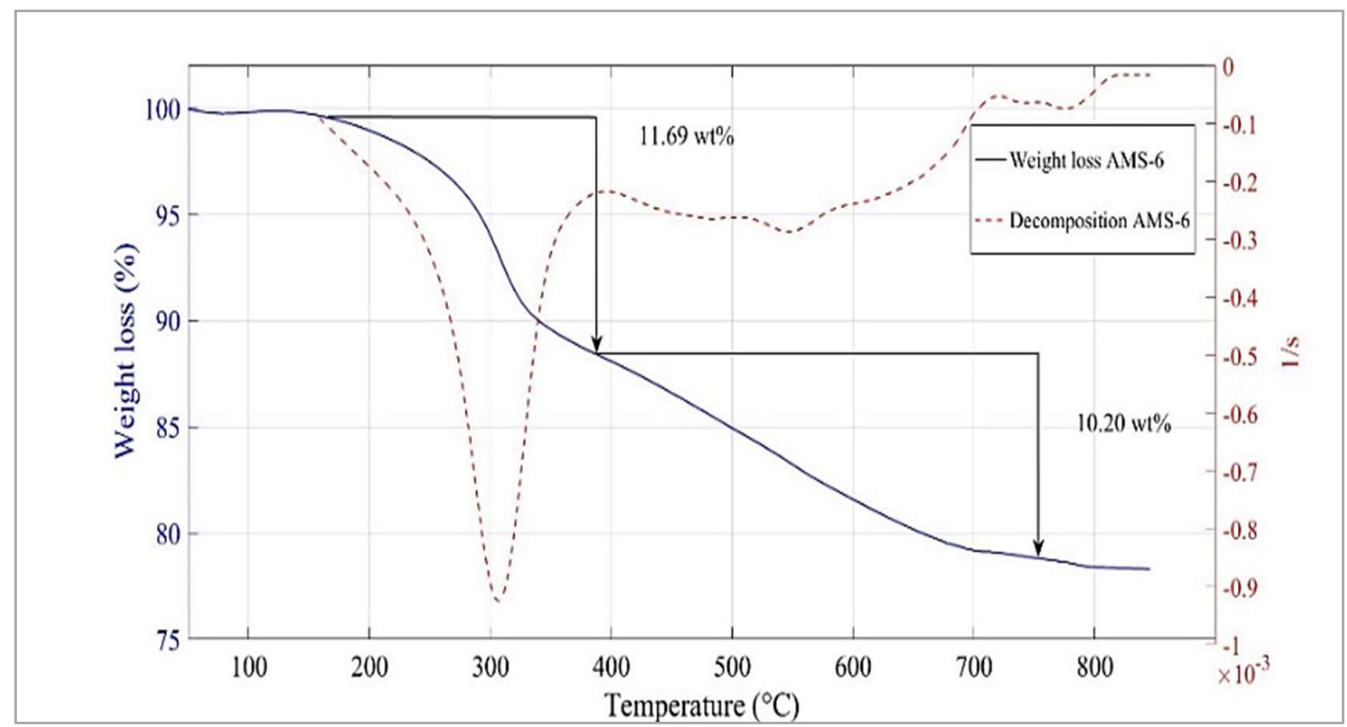

Figure A19. Thermogravimetric analysis of the unloaded AMS-6 nanoparticles sample. The thick blue line corresponds to the weight loss versus the temperature, and the dashed red line represents the first derivative from the thermogravimetric analysis (DTG) curve. The latter shows characteristic decomposition peaks, e.g. at $380^{\circ} \mathrm{C}$. The measurement was performed on the pure AMS- 6 to confirm the absence of any organic compounds that could block the pores, which were supposed to contain the loaded drug later. The peak of DTG at approximately $310^{\circ} \mathrm{C}$ corresponds to the loss (decomposition) of propyl amine functionalized groups. The analysis also revealed the incorporation of approximately $11.69 \mathrm{wt} \%$ of covalently bound propyl amine groups in the pores of the NPs. However, with $11.69 \mathrm{wt} \%$ the presence of amine groups was slightly elevated. A higher value would indicate a high amount of remaining amino groups, which would mean a blockage of the NPs pores. The remaining weight loss above $400^{\circ} \mathrm{C}$ was due to evaporating water (also known as extra framework water) caused by condensation of silanol groups. 


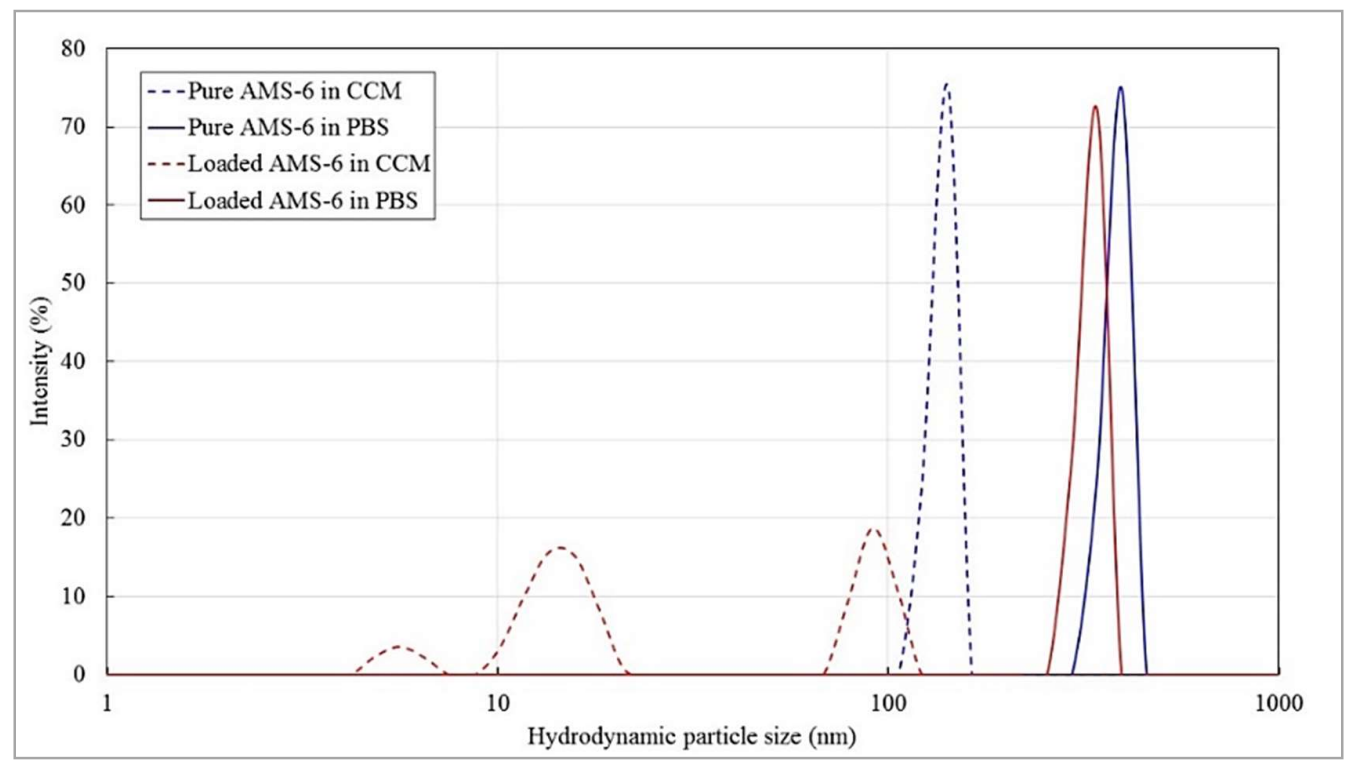

Figure A20. Hydrodynamic size distribution of pure and loaded AMS-6 NPs in complete culture medium (CCM) and in PBS. Note the variation of the particles' size distribution depending on the used dilution medium. 
A2.5.2. Effect of free and nanoformulated Doxorubicin on MDA-MB-231 cells in 2D and 3D (TECs) cultures

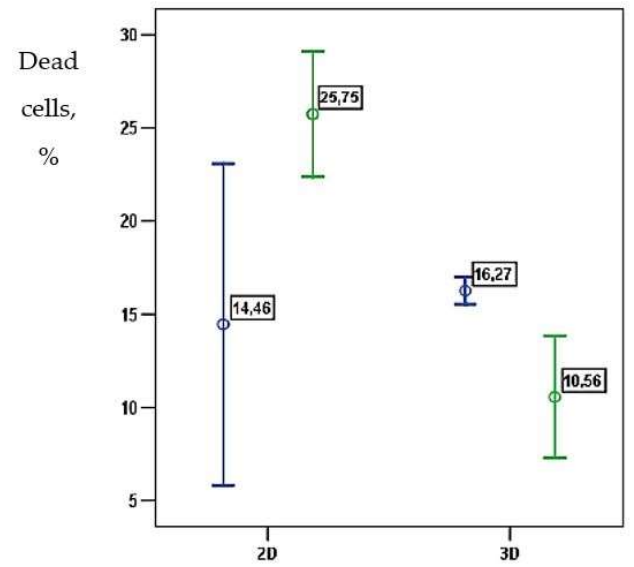

(a)

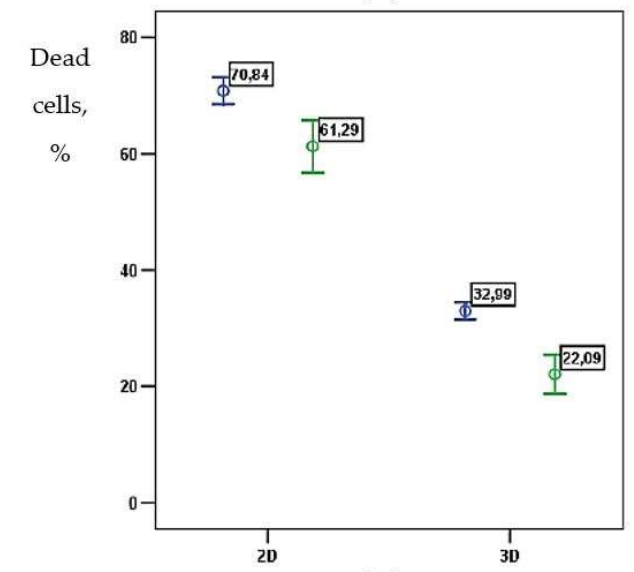

(c)

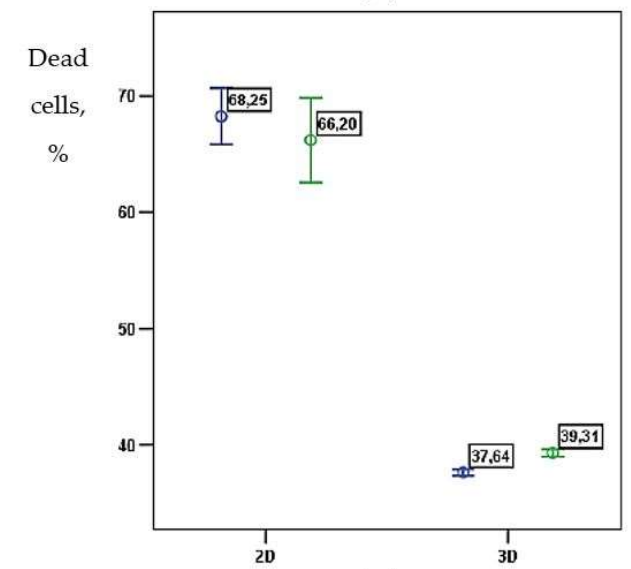

(e)

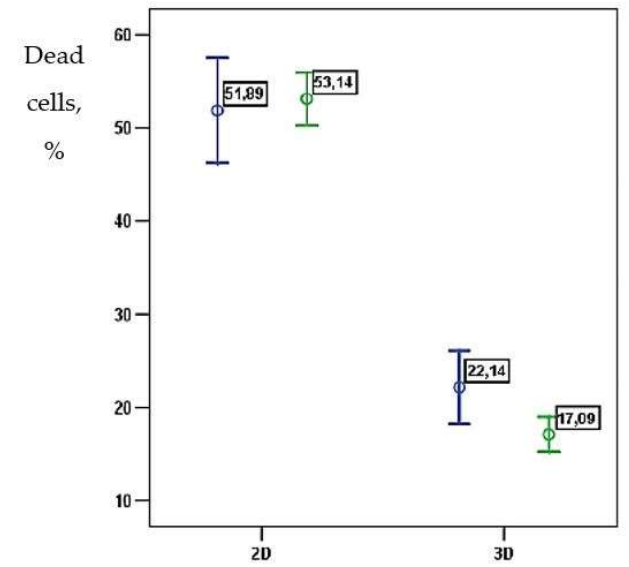

(b)

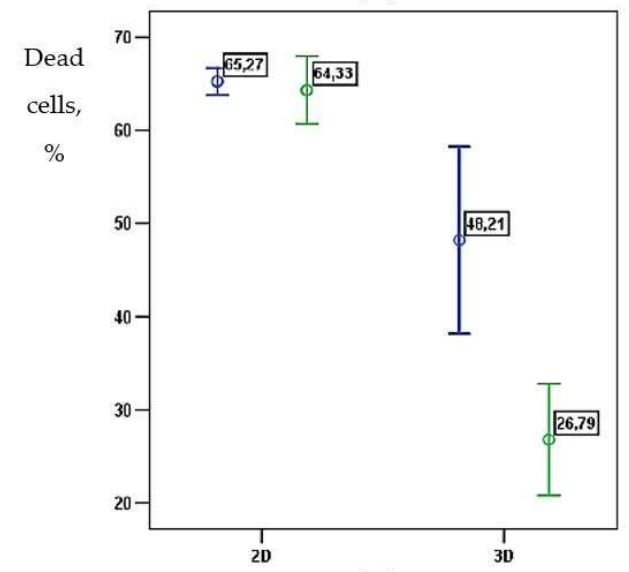

(d)

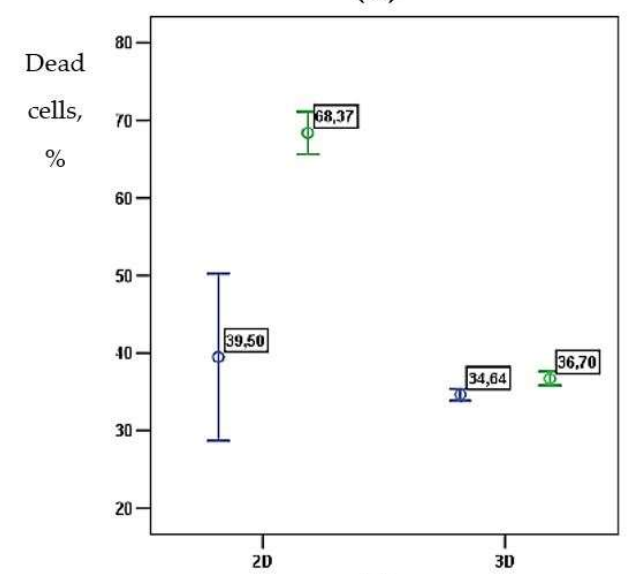

(f)

Figure A21. Intergroup comparisons of the cytotoxic effect of free and nanoformulated Dox in 2D culture of MDA-MB231 cells and 3D TECs after $36 \mathrm{~h}$ exposure. The mean ratio of dead cells after application of free Dox (blue) and AMS-6Dox (green), respectively, is shown for the following concentrations of Dox: (a) $0.1 \mu \mathrm{g} / \mathrm{mL}$; (b) $1 \mu \mathrm{g} / \mathrm{mL}$; (c) $3 \mu \mathrm{g} / \mathrm{mL}$; (d) 5 $\mu \mathrm{g} / \mathrm{mL}$; (e) $7 \mu \mathrm{g} / \mathrm{mL}$, and (f) $10 \mu \mathrm{g} / \mathrm{mL}$. Error bars show 95\% confidential intervals for the mean percent of dead cells. Data labels on the graphs also indicate the mean $\%$ of dead cells in the group. 
Table A6. The results of fitting a dose-response model to the measurements of cell viability. Estimated values of dose response model parameters for free Dox in 2D and 3D.

\begin{tabular}{ccccc}
\hline & \multicolumn{2}{c}{ 2D } & \multicolumn{2}{c}{ 3D } \\
\cline { 2 - 5 } & Estimated value & Standard error & Estimated value & Standard error \\
\hline Maximum effect, $\%$ & 64.85 & 2.83 & 34.34 & 2.71 \\
\hline EC50, $\mu \mathrm{g} / \mathrm{mL}$ & 0.27 & 0.09 & 2.40 & 0.41 \\
\hline Hill coefficient & 3.20 & 1.27 & 0.68 & 0.39 \\
\hline $\mathrm{IC} 50, \mu \mathrm{g} / \mathrm{mL}$ & 0.43 & 0.12 & $>10$ & - \\
\hline
\end{tabular}

Table A7. The results of fitting the dose-response model to the measurements of cell viability. Estimated values of dose response model parameters for AMS-6-Dox in 2D and 3D.

\begin{tabular}{ccccc}
\hline & \multicolumn{2}{c}{ 2D } & \multicolumn{2}{c}{ 3D } \\
\cline { 2 - 5 } & Estimated value & Standard error & Estimated value & Standard error \\
\hline Maximum effect, $\%$ & 63.06 & 1.97 & 40.53 & 5.47 \\
\hline EC50, $\mu \mathrm{g} / \mathrm{mL}$ & 0.26 & 0.06 & 2.38 & 0.86 \\
\hline Hill coefficient & 2.81 & 0.80 & 0.34 & 0.18 \\
\hline IC50, $\mu \mathrm{g} / \mathrm{mL}$ & 0.46 & 0.10 & $>10$ & - \\
\hline
\end{tabular}




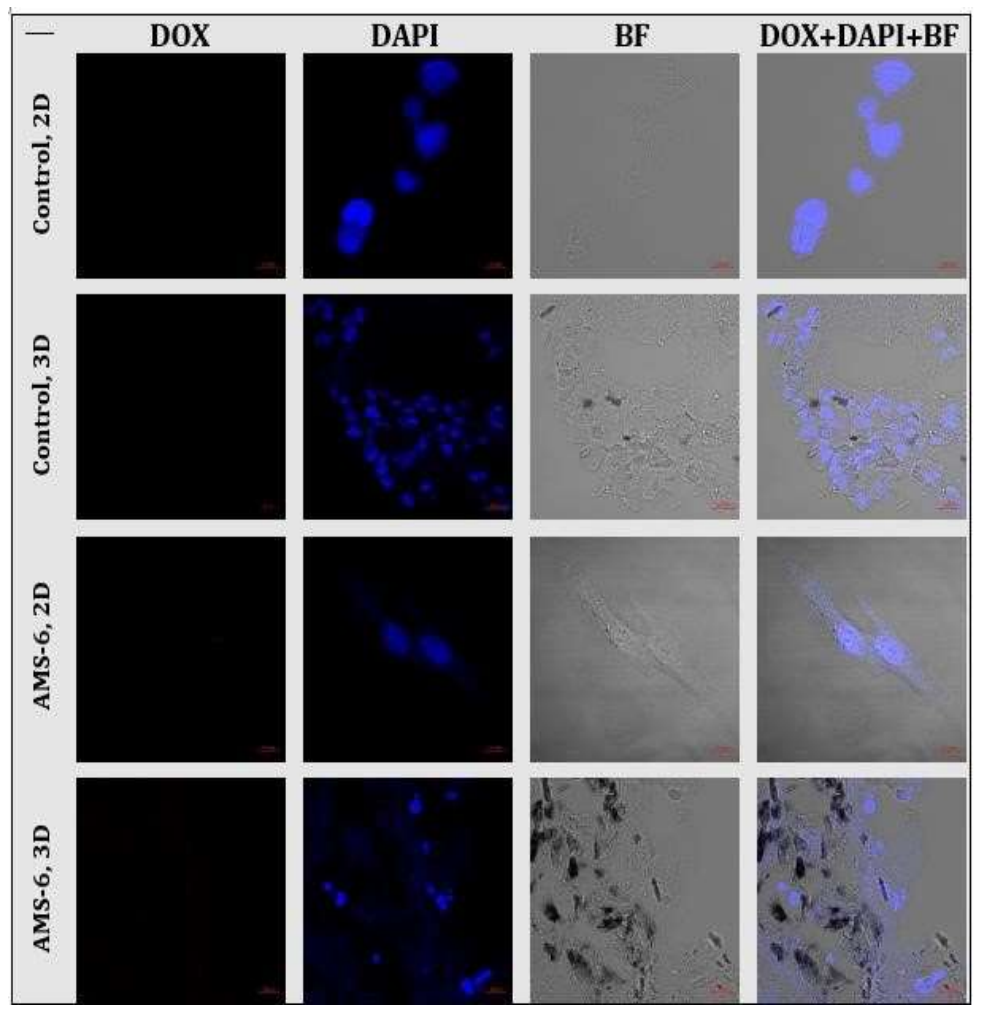

Figure A22. Laser-scanning confocal fluorescence microscopy images 2D and 3D TECs in vitro cultures of MDA-MB-231 cells incubated with complete culture medium (control 2D and 3D) and pure (not loaded with Dox) AMS-6 nanoparticles for $24 \mathrm{~h}$. Note the absence of Dox fluorescence in the red channel (Dox), the clear association of DAPI fluorescence staining with cell nuclei (blue channel, DAPI). AMS-6 concentration, $50 \mu \mathrm{g} / \mathrm{mL}$.
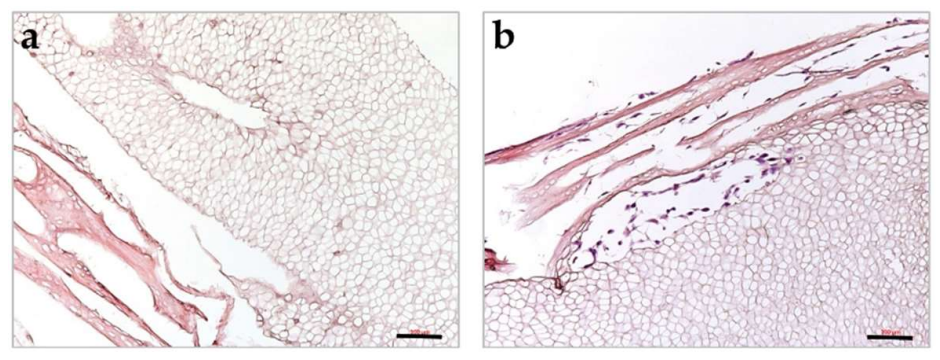

Figure A23. (a) Effect of iWO-DCL with an additional decalcination stage on CE tibial bone. (b) Recellularization of the bone AOSS with MDA-MB-231 cells. Staining with H\&E, scale bars 100 $\mu \mathrm{m}$. 


\section{References}

1. 1. Valastyan, S.; Weinberg, R.A. Tumor metastasis: molecular insights and evolving paradigms. Cell 2011, 147, 275-292, doi:10.1016/j.cell.2011.09.024.

2. 2. Dillekas, H.; Rogers, M.S.; Straume, O. Are $90 \%$ of deaths from cancer caused by metastases? Cancer Med 2019, 8, 55745576, doi:10.1002/cam4.2474.

3. 3. Riggio, A.I.; Varley, K.E.; Welm, A.L. The lingering mysteries of metastatic recurrence in breast cancer. British journal of cancer 2021, 124, 13-26, doi:10.1038/s41416-020-01161-4.

4. 4. Baeriswyl, V.; Christofori, G. The angiogenic switch in carcinogenesis. Semin Cancer Biol 2009, 19, 329-337, doi:10.1016/j.semcancer.2009.05.003.

5. 5. Nguyen, D.X.; Bos, P.D.; Massague, J. Metastasis: from dissemination to organ-specific colonization. Nat Rev Cancer 2009, 9, 274-284, doi:10.1038/nrc2622.

6. 6. Hanahan, D.; Folkman, J. Patterns and emerging mechanisms of the angiogenic switch during tumorigenesis. Cell 1996, 86, 353-364, doi:10.1016/s0092-8674(00)80108-7.

7. 7. Pantel, K.; Alix-Panabieres, C.; Riethdorf, S. Cancer micrometastases. Nat Rev Clin Oncol 2009, 6, 339-351, doi:10.1038/nrclinonc.2009.44.

8. 8. Lambert, A.W.; Pattabiraman, D.R.; Weinberg, R.A. Emerging Biological Principles of Metastasis. Cell 2017, 168, 670691, doi:10.1016/j.cell.2016.11.037.

9. 9. Holmgren, L.; O'Reilly, M.S.; Folkman, J. Dormancy of micrometastases: balanced proliferation and apoptosis in the presence of angiogenesis suppression. Nature medicine 1995, 1, 149-153, doi:10.1038/nm0295-149.

10. 10. Zhao, Z.; Zhu, X.; Cui, K.; Mancuso, J.; Federley, R.; Fischer, K.; Teng, G.; Mittal, V.; Gao, D.; Zhao, H.; et al. In Vivo Visualization and Characterization of Epithelial-Mesenchymal Transition in Breast Tumors. Cancer Res 2016, 76, 2094-2104, doi:10.1158/0008-5472.CAN-15-2662.

11. 11. Chen, W.; Hoffmann, A.D.; Liu, H.; Liu, X. Organotropism: new insights into molecular mechanisms of breast cancer metastasis. NPJ Precis Oncol 2018, 2, 4, doi:10.1038/s41698-018-0047-0.

12. 12. Nan, X.; Wang, J.; Liu, H.N.; Wong, S.T.C.; Zhao, H. Epithelial-Mesenchymal Plasticity in Organotropism Metastasis and Tumor Immune Escape. J Clin Med 2019, 8, 747, doi:10.3390/jcm8050747.

13. 13. Wei, S.; Siegal, G.P. Surviving at a distant site: The organotropism of metastatic breast cancer. Semin Diagn Pathol 2018, 35, 108-111, doi:10.1053/j.semdp.2017.11.008.

14. 14. Thiery, J.P. Epithelial-mesenchymal transitions in tumour progression. Nat Rev Cancer 2002, 2, 442-454, doi:10.1038/nrc822.

15. 15. Massague, J.; Obenauf, A.C. Metastatic colonization by circulating tumour cells. Nature 2016, 529, 298-306, doi:10.1038/nature17038.

16. 16. Cox, T.R. The matrix in cancer. Nat Rev Cancer 2021, doi:10.1038/s41568-020-00329-7.

17. 17. Gkretsi, V.; Stylianopoulos, T. Cell Adhesion and Matrix Stiffness: Coordinating Cancer Cell Invasion and Metastasis. Front Oncol 2018, 8, 145, doi:10.3389/fonc.2018.00145.

18. 18. Pathak, A.; Kumar, S. Independent regulation of tumor cell migration by matrix stiffness and confinement. Proceedings of the National Academy of Sciences of the United States of America 2012, 109, 10334-10339, doi:10.1073/pnas.1118073109.

19. 19. Guzman, A.; Ziperstein, M.J.; Kaufman, L.J. The effect of fibrillar matrix architecture on tumor cell invasion of physically challenging environments. Biomaterials 2014, 35, 6954-6963, doi:10.1016/j.biomaterials.2014.04.086.

20. 20. Guller, A.E.; Grebenyuk, P.N.; Shekhter, A.B.; Zvyagin, A.V.; Deyev, S.M. Bioreactor-Based Tumor Tissue Engineering. Acta Naturae 2016, 8, 44-58.

21. 21. Burdett, E.; Kasper, F.K.; Mikos, A.G.; Ludwig, J.A. Engineering tumors: a tissue engineering perspective in cancer biology. Tissue engineering. Part B, Reviews 2010, 16, 351-359, doi:10.1089/ten.TEB.2009.0676.

22. 22. Rijal, G.; Li, W. 3D scaffolds in breast cancer research. Biomaterials 2016, 81, 135-156, doi:10.1016/j.biomaterials.2015.12.016.

23. 23. Badylak, S.F. Decellularized allogeneic and xenogeneic tissue as a bioscaffold for regenerative medicine: factors that influence the host response. Ann Biomed Eng 2014, 42, 1517-1527, doi:10.1007/s10439-013-0963-7.

24. 24. Crapo, P.M.; Gilbert, T.W.; Badylak, S.F. An overview of tissue and whole organ decellularization processes. Biomaterials 2011, 32, 3233-3243, doi:10.1016/j.biomaterials.2011.01.057.

25. 25. Keane, T.J.; Swinehart, I.T.; Badylak, S.F. Methods of tissue decellularization used for preparation of biologic scaffolds and in vivo relevance. Methods 2015, doi:10.1016/j.ymeth.2015.03.005.

26. 26. Parmaksiz, M.; Dogan, A.; Odabas, S.; Elcin, A.E.; Elcin, Y.M. Clinical applications of decellularized extracellular matrices for tissue engineering and regenerative medicine. Biomedical materials 2016, 11, 022003, doi:10.1088/1748-6041/11/2/022003.

27. 27. Ferreira, L.P.; Gaspar, V.M.; Mano, J.F. Decellularized Extracellular Matrix for Bioengineering Physiomimetic 3D in Vitro Tumor Models. Trends Biotechnol 2020, 38, 1397-1414, doi:10.1016/j.tibtech.2020.04.006.

28. 28. Grey, J.F.E.; Campbell-Ritchie, A.; Everitt, N.M.; Fezovich, A.J.; Wheatley, S.P. The use of decellularised animal tissue to study disseminating cancer cells. Journal of cell science 2018, 132, jcs219907, doi:10.1242/jcs.219907.

29. 29. Shologu, N.; Szegezdi, E.; Lowery, A.; Kerin, M.; Pandit, A.; Zeugolis, D.I. Recreating complex pathophysiologies in vitro with extracellular matrix surrogates for anticancer therapeutics screening. Drug Discov Today 2016, doi:10.1016/j.drudis.2016.06.001. 
30. 30. Chakraborty, J.; Roy, S.; Ghosh, S. Regulation of decellularized matrix mediated immune response. Biomaterials science 2020, 8, 1194-1215, doi:10.1039/c9bm01780a.

31. 31. Ott, H.C.; Matthiesen, T.S.; Goh, S.K.; Black, L.D.; Kren, S.M.; Netoff, T.I.; Taylor, D.A. Perfusion-decellularized matrix: using nature's platform to engineer a bioartificial heart. Nature medicine 2008, 14, 213-221, doi:10.1038/nm1684.

32. 32. Mayorca-Guiliani, A.E.; Madsen, C.D.; Cox, T.R.; Horton, E.R.; Venning, F.A.; Erler, J.T. ISDoT: in situ decellularization of tissues for high-resolution imaging and proteomic analysis of native extracellular matrix. Nature medicine 2017, 23, 890-898, doi:10.1038/nm.4352.

33. 33. Park, K.M.; Woo, H.M. Systemic Decellularization for Multi-organ Scaffolds in Rats. Transplantation proceedings 2012, 44, 1151-1154, doi:http://dx.doi.org/10.1016/j.transproceed.2012.03.017.

34. 34. Swinehart, I.T.; Badylak, S.F. Extracellular matrix bioscaffolds in tissue remodeling and morphogenesis. Developmental dynamics : an official publication of the American Association of Anatomists 2016, 245, 351-360, doi:10.1002/dvdy.24379.

35. 35. Shekhter, A.B.; Guller, A.E.; Istranov, L.P.; Istranova, E.V.; Butnaru, D.V.; Vinarov, A.Z.; Zakharkina, O.L.; Kurkov, A.V.; Kantimerov, D.F.; Antonov, E.N.; et al. [Morphology of collagen matrices for tissue engineering (biocompatibility, biodegradation, tissue response)]. Arkh Patol 2015, 77, 29-38, doi:10.17116/patol201577629-38.

36. 36. Marques, C.F.; Diogo, G.S.; Pina, S.; Oliveira, J.M.; Silva, T.H.; Reis, R.L. Collagen-based bioinks for hard tissue engineering applications: a comprehensive review. J Mater Sci Mater Med 2019, 30, 32, doi:10.1007/s10856-019-6234-x.

37. 37. Liang, J.; Yi, P.; Wang, X.; Huang, F.; Luan, X.; Zhao, Z.; Liu, C. Acellular matrix hydrogel for repair of the temporomandibular joint disc. Journal of biomedical materials research. Part B, Applied biomaterials 2020, n/a, doi:10.1002/jbm.b.34629.

38. 38. Hynes, R.O. The extracellular matrix: not just pretty fibrils. Science 2009, 326, 1216-1219, doi:10.1126/science.1176009.

39. 39. Hutter, H.; Vogel, B.E.; Plenefisch, J.D.; Norris, C.R.; Proenca, R.B.; Spieth, J.; Guo, C.; Mastwal, S.; Zhu, X.; Scheel, J.; et al. Conservation and novelty in the evolution of cell adhesion and extracellular matrix genes. Science 2000, 287, 989-994, doi:10.1126/science.287.5455.989.

40. 40. Tottey, S.; Johnson, S.A.; Crapo, P.M.; Reing, J.E.; Zhang, L.; Jiang, H.; Medberry, C.J.; Reines, B.; Badylak, S.F. The effect of source animal age upon extracellular matrix scaffold properties. Biomaterials 2011, 32, 128-136, doi:10.1016/j.biomaterials.2010.09.006.

41. 41. Aamodt, J.M.; Grainger, D.W. Extracellular matrix-based biomaterial scaffolds and the host response. Biomaterials 2016, 86, 68-82, doi:10.1016/j.biomaterials.2016.02.003.

42. 42. Morris, A.H.; Stamer, D.K.; Kyriakides, T.R. The host response to naturally-derived extracellular matrix biomaterials. Semin Immunol 2017, 29, 72-91, doi:10.1016/j.smim.2017.01.002.

43. 43. McNeal, W.D.; Fletcher, D.L.; Buhr, R.J. Effects of stunning and decapitation on broiler activity during bleeding, blood loss, carcass, and breast meat quality. Poult Sci 2003, 82, 163-168, doi:10.1093/ps/82.1.163.

44. 44. Guller, A.; Trusova, I.; Petersen, E.; Shekhter, A.; Kurkov, A.; Qian, Y.; Zvyagin, A. Acellular organ scaffolds for tumor tissue engineering. In Proceedings of the Micro+Nano Materials, Devices, and Systems, Sydney, New South Wales, Australia, December 06, 2015, 2015; pp. 96684G-96684G-96689.

45. 45. Khabir, Z.; Guller, A.E.; Rozova, V.S.; Liang, L.; Lai, Y.J.; Goldys, E.M.; Hu, H.; Vickery, K.; Zvyagin, A.V. Tracing upconversion nanoparticle penetration in human skin. Colloids and surfaces. B, Biointerfaces 2019, 184, 110480, doi:10.1016/j.colsurfb.2019.110480.

46. 46. Ibidi, G. Liv e/d ead staining with FDA and PI. Application Note \# 33. Available online: https://ibidi.com/img/cms/support/AN/AN33_Live_Dead_staining_with_FDA_and_PI.pdf (accessed on

47. 47. Kotova, S.L.; Timashev, P.S.; Guller, A.E.; Shekhter, A.B.; Misurkin, P.I.; Bagratashvili, V.N.; Solovieva, A.B. Collagen structure deterioration in the skin of patients with pelvic organ prolapse determined by atomic force microscopy. Microscopy and microanalysis : the official journal of Microscopy Society of America, Microbeam Analysis Society, Microscopical Society of Canada 2015, 21, 324-333, doi:10.1017/S1431927615000148.

48. 48. Mosmann, T. Rapid colorimetric assay for cellular growth and survival: application to proliferation and cytotoxicity assays. Journal of immunological methods 1983, 65, 55-63, doi:10.1016/0022-1759(83)90303-4.

49. 49. Nowak-Sliwinska, P.; Segura, T.; Iruela-Arispe, M.L. The chicken chorioallantoic membrane model in biology, medicine and bioengineering. Angiogenesis 2014, 17, 779-804, doi:10.1007/s10456-014-9440-7.

50. 50. Steger, C. An unbiased detector of curvilinear structures. Ieee Transactions on Pattern Analysis and Machine Intelligence 1998, 20, 113-125, doi:Doi 10.1109/34.659930.

51. 51. Carpentier, G. Angiogenesis Analyzer for ImageJ, available online: http://imagej.nih.gov/ij/macros/toolsets/Angiogenesis\%20Analyzer.txt. Available online: http://image.bio.methods.free.fr/ImageJ/IMG/png/binary-huvec-fluo-tree-tr.png (accessed on

52. 52. Garcia-Bennett, A.E.; Miyasaka, K.; Terasaki, O.; Che, S.N. Structural solution of mesocaged material AMS-8. Chemistry of Materials 2004, 16, 3597-3605, doi:10.1021/cm049398e.

53. 53. Garcia-Bennett, A.E.; Terasaki, O.; Che, S.; Tatsumi, T. Structural investigations of AMS-n mesoporous materials by transmission electron microscopy. Chemistry of Materials 2004, 16, 813-821, doi:10.1021/cm035074z.

54. 54. Brunauer, S.; Emmett, P.H.; Teller, E. Adsorption of gases in multimolecular layers. Journal of the American Chemical Society 1938, 60, 309-319, doi:DOI 10.1021/ja01269a023.

55. 55. Uygun, B.E.; Soto-Gutierrez, A.; Yagi, H.; Izamis, M.L.; Guzzardi, M.A.; Shulman, C.; Milwid, J.; Kobayashi, N.; Tilles, A.; Berthiaume, F.; et al. Organ reengineering through development of a transplantable recellularized liver graft using decellularized liver matrix. Nature medicine 2010, 16, 814-820, doi:10.1038/nm.2170. 
56. 56. Zhou, P.; Lessa, N.; Estrada, D.C.; Severson, E.B.; Lingala, S.; Zern, M.A.; Nolta, J.A.; Wu, J. Decellularized liver matrix as a carrier for the transplantation of human fetal and primary hepatocytes in mice. Liver Transpl 2011, 17, 418-427, doi:10.1002/lt.22270.

57. 57. Kajbafzadeh, A.M.; Javan-Farazmand, N.; Monajemzadeh, M.; Baghayee, A. Determining the optimal decellularization and sterilization protocol for preparing a tissue scaffold of a human-sized liver tissue. Tissue engineering. Part C, Methods 2013, 19, 642-651, doi:10.1089/ten.TEC.2012.0334.

58. 58. Witasp, E.; Kupferschmidt, N.; Bengtsson, L.; Hultenby, K.; Smedman, C.; Paulie, S.; Garcia-Bennett, A.E.; Fadeel, B. Efficient internalization of mesoporous silica particles of different sizes by primary human macrophages without impairment of macrophage clearance of apoptotic or antibody-opsonized target cells. Toxicol Appl Pharmacol 2009, 239, 306-319, doi:10.1016/j.taap.2009.06.011.

59. 59. Tizard, M.L.; Jenkins, K.A.; Cooper, C.A.; Woodcock, M.E.; Challagulla, A.; Doran, T.J. Potential benefits of gene editing for the future of poultry farming. Transgenic Res 2019, 28, 87-92, doi:10.1007/s11248-019-00139-0.

60. 60. Clement, S.; Guller, A.; Mahbub, S.B.; Goldys, E.M. Oxygen-Carrying Polymer Nanoconstructs for Radiodynamic Therapy of Deep Hypoxic Malignant Tumors. Biomedicines 2021, 9, 322, doi:10.3390/biomedicines9030322.

61. 61. Baiguera, S.; Macchiarini, P.; Ribatti, D. Chorioallantoic membrane for in vivo investigation of tissue-engineered construct biocompatibility. Journal of biomedical materials research. Part B, Applied biomaterials 2012, 100, 1425-1434, doi:10.1002/jbm.b.32653.

62. 62. Ribatti, D. The chick embryo chorioallantoic membrane (CAM). A multifaceted experimental model. Mech Dev 2016, 141, 70-77, doi:10.1016/j.mod.2016.05.003.

63. 63. Lu, X.; Kang, Y. Organotropism of breast cancer metastasis. J Mammary Gland Biol Neoplasia 2007, 12, 153-162, doi:10.1007/s10911-007-9047-3.

64. 64. Foulkes, W.D.; Smith, I.E.; Reis-Filho, J.S. Triple-negative breast cancer. The New England journal of medicine 2010, 363, 1938-1948, doi:10.1056/NEJMra1001389.

65. 65. Carey, L.; Winer, E.; Viale, G.; Cameron, D.; Gianni, L. Triple-negative breast cancer: disease entity or title of convenience? Nat Rev Clin Oncol 2010, 7, 683-692, doi:10.1038/nrclinonc.2010.154.

66. 66. Yao, H.; He, G.; Yan, S.; Chen, C.; Song, L.; Rosol, T.J.; Deng, X. Triple-negative breast cancer: is there a treatment on the horizon? Oncotarget 2017, 8, 1913-1924, doi:10.18632/oncotarget.12284.

67. 67. Disibio, G.; French, S.W. Metastatic patterns of cancers: results from a large autopsy study. Arch Pathol Lab Med 2008, 132, 931-939, doi:10.1043/1543-2165(2008)132[931:MPOCRF]2.0.CO;2

68. 10.5858/2008-132-931-MPOCRF.

69. 68. Wong, G.L.; Abu Jalboush, S.; Lo, H.W. Exosomal MicroRNAs and Organotropism in Breast Cancer Metastasis. Cancers (Basel) 2020, 12, 1827, doi:10.3390/cancers12071827.

70. 69. Dent, R.; Trudeau, M.; Pritchard, K.I.; Hanna, W.M.; Kahn, H.K.; Sawka, C.A.; Lickley, L.A.; Rawlinson, E.; Sun, P.; Narod, S.A. Triple-negative breast cancer: clinical features and patterns of recurrence. Clin Cancer Res 2007, 13, 4429-4434, doi:10.1158/1078-0432.CCR-06-3045.

71. 70. Wei, S.; Siegal, G.P. Metastatic Organotropism: An Intrinsic Property of Breast Cancer Molecular Subtypes. Adv Anat Pathol 2017, 24, 78-81, doi:10.1097/PAP.0000000000000140.

72. 71. Khoo, B.L.; Lee, S.C.; Kumar, P.; Tan, T.Z.; Warkiani, M.E.; Ow, S.G.; Nandi, S.; Lim, C.T.; Thiery, J.P. Short-term expansion of breast circulating cancer cells predicts response to anti-cancer therapy. Oncotarget 2015, 6, 15578-15593, doi:10.18632/oncotarget.3903.

73. 72. Xiong, G.; Flynn, T.J.; Chen, J.; Trinkle, C.; Xu, R. Development of an ex vivo breast cancer lung colonization model utilizing a decellularized lung matrix. Integrative biology : quantitative biosciences from nano to macro 2015, 7, 1518-1525, doi:10.1039/c5ib00157a.

74. 73. Tsilimigras, D.I.; Brodt, P.; Clavien, P.-A.; Muschel, R.J.; D’Angelica, M.I.; Endo, I.; Parks, R.W.; Doyle, M.; de Santibañes, E.; Pawlik, T.M. Liver metastases. Nature Reviews Disease Primers 2021, 7, 27, doi:10.1038/s41572-021-00261-6.

75. 74. Ma, R.; Feng, Y.; Lin, S.; Chen, J.; Lin, H.; Liang, X.; Zheng, H.; Cai, X. Mechanisms involved in breast cancer liver metastasis. J Transl Med 2015, 13, 64, doi:10.1186/s12967-015-0425-0.

76. 75. Rosenow, F.; Ossig, R.; Thormeyer, D.; Gasmann, P.; Schlüter, K.; Brunner, G.; Haier, J.; Eble, J.A. Integrins as Antimetastatic Targets of RGD-Independent Snake Venom Components in Liver Metastasis. Neoplasia (New York, N.Y.) 2008, 10, 168176.

77. 76. Haier, J. An Intravital Model to Monitor Steps of Metastatic Tumor Cell Adhesion Within the Hepatic Microcirculation. Journal of Gastrointestinal Surgery 2003, 7, 507-515, doi:10.1016/s1091-255x(03)00023-4.

78. 77. Roos, E.; Dingemans, K.P.; Van de Pavert, I.V.; Van den Bergh-Weerman, M.A. Mammary-carcinoma cells in mouse liver: infiltration of liver tissue and interaction with Kupffer cells. British journal of cancer 1978, 38, 88-99, doi:10.1038/bjc.1978.167.

79. 78. Liang, Y.; Zhang, H.; Song, X.; Yang, Q. Metastatic heterogeneity of breast cancer: Molecular mechanism and potential therapeutic targets. Semin Cancer Biol 2020, 60, 14-27, doi:10.1016/j.semcancer.2019.08.012.

80. 79. Martinez-Hernandez, A.; Amenta, P.S. The extracellular matrix in hepatic regeneration. FASEB journal : official publication of the Federation of American Societies for Experimental Biology 1995, 9, 1401-1410, doi:10.1096/fasebj.9.14.7589981. 
81. 80. Tabaries, S.; Dong, Z.; Annis, M.G.; Omeroglu, A.; Pepin, F.; Ouellet, V.; Russo, C.; Hassanain, M.; Metrakos, P.; Diaz, Z.; et al. Claudin-2 is selectively enriched in and promotes the formation of breast cancer liver metastases through engagement of integrin complexes. Oncogene 2011, 30, 1318-1328, doi:10.1038/onc.2010.518.

82. 81. Ishii, S.; Mizoi, T.; Kawano, K.; Cay, O.; Thomas, P.; Nachman, A.; Ford, R.; Shoji, Y.; Kruskal, J.B.; Steele, G., Jr.; et al. Implantation of human colorectal carcinoma cells in the liver studied by in vivo fluorescence videomicroscopy. Clinical \& experimental metastasis 1996, 14, 153-164, doi:10.1007/BF00121212.

83. 82. Barkan, D.; Green, J.E.; Chambers, A.F. Extracellular matrix: a gatekeeper in the transition from dormancy to metastatic growth. Eur J Cancer 2010, 46, 1181-1188, doi:10.1016/j.ejca.2010.02.027.

84. 83. Seo, B.R.; DelNero, P.; Fischbach, C. In vitro models of tumor vessels and matrix: engineering approaches to investigate transport limitations and drug delivery in cancer. Adv Drug Deliv Rev 2014, 69-70, 205-216, doi:10.1016/j.addr.2013.11.011.

85. 84. Faulk, D.M.; Wildemann, J.D.; Badylak, S.F. Decellularization and cell seeding of whole liver biologic scaffolds composed of extracellular matrix. J Clin Exp Hepatol 2015, 5, 69-80, doi:10.1016/j.jceh.2014.03.043.

86. 85. Rozova, V.S.; Anwer, A.G.; Guller, A.E.; Es, H.A.; Khabir, Z.; Sokolova, A.I.; Gavrilov, M.U.; Goldys, E.M.; Warkiani, M.E.; Thiery, J.P.; et al. Machine learning reveals mesenchymal breast carcinoma cell adaptation in response to matrix stiffness. PLoS Comput Biol 2021, 17, e1009193, doi:10.1371/journal.pcbi.1009193.

87. 86. Guimarães, C.F.; Gasperini, L.; Marques, A.P.; Reis, R.L. The stiffness of living tissues and its implications for tissue engineering. Nature Reviews Materials 2020, 5, 351-370, doi:10.1038/s41578-019-0169-1.

88. 87. Yarmenitis, S.D.; Kalogeropoulou, C.P.; Hatjikondi, O.; Ravazoula, P.; Petsas, T.; Siamblis, D.; Kalfarentzos, F. An experimental approach of the Doppler perfusion index of the liver in detecting occult hepatic metastases: histological findings related to the hemodynamic measurements in Wistar rats. Eur Radiol 2000, 10, 417-424, doi:10.1007/s003300050068.

89. 88. Alzubi, M.A.; Sohal, S.S.; Sriram, M.; Turner, T.H.; Zot, P.; Idowu, M.; Harrell, J.C. Quantitative assessment of breast cancer liver metastasis expansion with patient-derived xenografts. Clinical \& experimental metastasis 2019, 36, 257-269, doi:10.1007/s10585-019-09968-z.

90. 89. Clark, A.M.; Kumar, M.P.; Wheeler, S.E.; Young, C.L.; Venkataramanan, R.; Stolz, D.B.; Griffith, L.G.; Lauffenburger, D.A.; Wells, A. A Model of Dormant-Emergent Metastatic Breast Cancer Progression Enabling Exploration of Biomarker Signatures. Mol Cell Proteomics 2018, 17, 619-630, doi:10.1074/mcp.RA117.000370.

91. 90. Kingston, B.R.; Syed, A.M.; Ngai, J.; Sindhwani, S.; Chan, W.C.W. Assessing micrometastases as a target for nanoparticles using 3D microscopy and machine learning. Proceedings of the National Academy of Sciences of the United States of America 2019, 116, 14937-14946, doi:10.1073/pnas.1907646116.

92. 91. Friedl, P.; Wolf, K. Tumour-cell invasion and migration: diversity and escape mechanisms. Nat Rev Cancer 2003, 3, 362374, doi:10.1038/nrc1075.

93. 92. Liu, Y.J.; Le Berre, M.; Lautenschlaeger, F.; Maiuri, P.; Callan-Jones, A.; Heuze, M.; Takaki, T.; Voituriez, R.; Piel, M. Confinement and low adhesion induce fast amoeboid migration of slow mesenchymal cells. Cell 2015, 160, 659-672, doi:10.1016/j.cell.2015.01.007.

94. 93. Holtkamp, N.; Afanasieva, A.; Elstner, A.; van Landeghem, F.K.; Konneker, M.; Kuhn, S.A.; Kettenmann, H.; von Deimling, A. Brain slice invasion model reveals genes differentially regulated in glioma invasion. Biochem Biophys Res Commun 2005, 336, 1227-1233, doi:10.1016/j.bbrc.2005.08.253.

95. 94. Friedl, P. Prespecification and plasticity: shifting mechanisms of cell migration. Curr Opin Cell Biol 2004, 16, 14-23, doi:10.1016/j.ceb.2003.11.001.

96. 95. McClelland, R.; Wauthier, E.; Uronis, J.; Reid, L. Gradients in the liver's extracellular matrix chemistry from periportal to pericentral zones: influence on human hepatic progenitors. Tissue engineering. Part A 2008, 14, 59-70, doi:10.1089/ten.a.2007.0058

97. 96. Reid, L.M.; Fiorino, A.S.; Sigal, S.H.; Brill, S.; Holst, P.A. Extracellular matrix gradients in the space of Disse: relevance to liver biology. Hepatology 1992, 15, 1198-1203, doi:10.1002/hep.1840150635.

98. 97. Ling, W.; Lu, Q.; Lu, C.; Quan, J.; Ma, L.; Li, J.; He, D.; Liu, J.; Yang, J.; Wen, T.; et al. Effects of vascularity and differentiation of hepatocellular carcinoma on tumor and liver stiffness: in vivo and in vitro studies. Ultrasound in medicine \& biology 2014, 40, 739-746, doi:10.1016/j.ultrasmedbio.2013.08.011.

99. 98. Zaman, M.H.; Trapani, L.M.; Sieminski, A.L.; Mackellar, D.; Gong, H.; Kamm, R.D.; Wells, A.; Lauffenburger, D.A.; Matsudaira, P. Migration of tumor cells in 3D matrices is governed by matrix stiffness along with cell-matrix adhesion and proteolysis. Proceedings of the National Academy of Sciences of the United States of America 2006, 103, 10889-10894, doi:10.1073/pnas.0604460103.

100. 99. Schrader, J.; Gordon-Walker, T.T.; Aucott, R.L.; van Deemter, M.; Quaas, A.; Walsh, S.; Benten, D.; Forbes, S.J.; Wells, R.G.; Iredale, J.P. Matrix stiffness modulates proliferation, chemotherapeutic response, and dormancy in hepatocellular carcinoma cells. Hepatology 2011, 53, 1192-1205, doi:10.1002/hep.24108.

101. 100. Ranamukhaarachchi, S.K.; Modi, R.N.; Han, A.; Velez, D.O.; Kumar, A.; Engler, A.J.; Fraley, S.I. Macromolecular crowding tunes 3D collagen architecture and cell morphogenesis. Biomaterials science 2019, 7, 618-633, doi:10.1039/c8bm01188e.

102. 101. Herrera-Perez, M.; Voytik-Harbin, S.L.; Rickus, J.L. Extracellular Matrix Properties Regulate the Migratory Response of Glioblastoma Stem Cells in Three-Dimensional Culture. Tissue engineering. Part A 2015, 21, 2572-2582, doi:10.1089/ten.TEA.2014.0504.

103. 102. Viji Babu, P.K.; Rianna, C.; Mirastschijski, U.; Radmacher, M. Nano-mechanical mapping of interdependent cell and ECM mechanics by AFM force spectroscopy. Scientific reports 2019, 9, 12317, doi:10.1038/s41598-019-48566-7. 
104. 103. Grigore, A.D.; Jolly, M.K.; Jia, D.; Farach-Carson, M.C.; Levine, H. Tumor Budding: The Name is EMT. Partial EMT. Journal of Clinical Medicine 2016, 5, 51.

105. 104. Spratt, J.A.; von Fournier, D.; Spratt, J.S.; Weber, E.E. Decelerating growth and human breast cancer. Cancer 1993, 71, 2013-2019, doi:10.1002/1097-0142(19930315)71:6<2013::aid-cncr2820710615>3.0.co;2-v.

106. 105. Tsoularis, A.; Wallace, J. Analysis of logistic growth models. Math Biosci 2002, 179, 21-55, doi:10.1016/s00255564(02)00096-2.

107. 106. Singh, M.; Mukundan, S.; Jaramillo, M.; Oesterreich, S.; Sant, S. Three-Dimensional Breast Cancer Models Mimic Hallmarks of Size-Induced Tumor Progression. Cancer Res 2016, 76, 3732-3743, doi:10.1158/0008-5472.CAN-15-2304.

108. 107. Schlatter, P.; Konig, M.F.; Karlsson, L.M.; Burri, P.H. Quantitative study of intussusceptive capillary growth in the chorioallantoic membrane (CAM) of the chicken embryo. Microvascular research 1997, 54, 65-73, doi:10.1006/mvre.1997.2022.

109. 108. Hillen, F.; Griffioen, A.W. Tumour vascularization: sprouting angiogenesis and beyond. Cancer Metastasis Rev 2007, 26, 489-502, doi:10.1007/s10555-007-9094-7.

110. 109. Fischbach, C.; Chen, R.; Matsumoto, T.; Schmelzle, T.; Brugge, J.S.; Polverini, P.J.; Mooney, D.J. Engineering tumors with 3D scaffolds. Nat Methods 2007, 4, 855-860, doi:10.1038/nmeth1085.

111. 110. Hamburger, V.; Hamilton, H.L. A series of normal stages in the development of the chick embryo. J Morphol 1951, 88, 49-92, doi:doi:10.1002/jmor.1050880104.

112. 111. Atluri, R.; Hedin, N.; Garcia-Bennett, A.E. Hydrothermal phase transformation of bicontinuous cubic mesoporous material AMS-6. Chemistry of Materials 2008, 20, 3857-3866, doi:10.1021/cm702440n.

113. 112. Ribatti, D.; Conconi, M.T.; Nico, B.; Baiguera, S.; Corsi, P.; Parnigotto, P.P.; Nussdorfer, G.G. Angiogenic response induced by acellular brain scaffolds grafted onto the chick embryo chorioallantoic membrane. Brain research 2003, 989, 9-15, doi:10.1016/s0006-8993(03)03225-6.

114. 113. Hanahan, D.; Weinberg, R.A. Hallmarks of cancer: the next generation. Cell 2011, 144, 646-674, doi:10.1016/j.cell.2011.02.013.

115. 114. Mak, K.M.; Png, C.Y.; Lee, D.J. Type V Collagen in Health, Disease, and Fibrosis. Anatomical record 2016, 299, 613-629, doi:10.1002/ar.23330.

116. 115. Blacher, S.; Devy, L.; Hlushchuk, R.; Larger, E.; Lamandé, N.; Burri, P.; Corvol, P.; Djonov, V.; Foidart, J.-M.; Noël, A. Quantification of Angiogenesis in the Chicken Chorioallantoic Membrane (Cam). Image Analysis \& Stereology 2011, 23, 169180, doi:10.5566/ias.v24.p169-180.

117. 116. Doukas, C.N.; Maglogiannis, I.; Chatziioannou, A.A. Computer-supported angiogenesis quantification using image analysis and statistical averaging. IEEE Trans Inf Technol Biomed 2008, 12, 650-657, doi:10.1109/TITB.2008.926463. 118. 